Portland State University

PDXScholar

Fall 11-8-2018

\title{
Carbon-Supported Transition Metal Nanoparticles for Catalytic and Electromagnetic Applications
}

Kavita Meduri

Portland State University

Follow this and additional works at: https://pdxscholar.library.pdx.edu/open_access_etds

Part of the Nanoscience and Nanotechnology Commons Let us know how access to this document benefits you.

\section{Recommended Citation}

Meduri, Kavita, "Carbon-Supported Transition Metal Nanoparticles for Catalytic and Electromagnetic Applications" (2018). Dissertations and Theses. Paper 4639.

https://doi.org/10.15760/etd.6523

This Dissertation is brought to you for free and open access. It has been accepted for inclusion in Dissertations and Theses by an authorized administrator of PDXScholar. Please contact us if we can make this document more accessible: pdxscholar@pdx.edu. 
Carbon-Supported Transition Metal Nanoparticles for Catalytic and Electromagnetic Applications

by

Kavita Meduri

A dissertation submitted in partial fulfillment of the requirements for the degree of

\author{
Doctor of Philosophy \\ in \\ Mechanical Engineering
}
Dissertation Committee:
Jun Jiao, Chair
Paul G. Tratnyek
Lemmy Meekisho
Dacian Daescu

\author{
Portland State University \\ 2018
}


(C) 2018 Kavita Meduri 


\begin{abstract}
Recently, there has been growing interest in using transition metals (TM) for catalytic and electromagnetic applications, due to the ability of TMs to form stable compounds in multiple oxidation states. In this research, the focus has been on the synthesis and characterization of carbon-supported TM nanoparticles (NPs), specifically palladium (Pd) and gold $(\mathrm{Au}) \mathrm{NPs}$, for catalytic applications, and transition metal oxides (TMO) NPs, specifically $\mathrm{Fe}_{3} \mathrm{O}_{4}$ NPs for electromagnetic applications. Carbon supports have several advantages, such as enabling even distribution of particles, offering large specific surface area with excellent electron conductivity, and relative chemical inertness.
\end{abstract}

In this dissertation, for catalytic applications, emphasis was on removal of trichloroethylene (TCE) from groundwater. For this application, carbon-supported Pd/Au NP catalysts were developed. Pd was chosen because it is more active, stable and selective for desired end-products, and Au has shown to be a good promotor of Pd's catalytic activity. Often, commercially available Pd-based catalysts are made using harsh chemicals, which can be harmful to the environment. Here, an environmentally friendly process with aspects of green chemistry was developed to produce carbon-supported $\mathrm{Pd} / \mathrm{Au} \mathrm{NP}$ catalysts. This process uses a combination of sonochemistry and solvothermal syntheses. The carefully designed carbon-supported Pd/Au NP catalyst material was systematically characterized, tested against TCE, and optimized for increased rate of removal of TCE. Electron microscopy and spectroscopy techniques were used to study the material including structure, configuration and oxidative state. The Pd/Au NPs were found mainly to form clusters with an aggregate-Pd $\mathrm{d}_{\text {Shell }} \mathrm{Au}_{\text {Core }}$ structure. Using state-of-the-art direct 
detection with electron energy loss spectroscopy, the Pd NPs were found to have an oxidative state of zero (0). The formation of the catalyst material was studied in detail by varying several synthesis parameters including type of solvent, sonication time, synthesis temperature etc. The most optimized catalyst was found remove TCE at double the rate of corresponding commercial Pd-based catalysts in a hydrogen headspace. This material was found to catalyze the removal of TCE via traditional hydrodehalogenation and shows promise for the removal of other contaminants such as trichloropropane (TCP), carbon tetrachloride (CT).

This green approach to make and optimize TM materials for specific applications was extended to TMOs, specifically magnetite $\left(\mathrm{Fe}_{3} \mathrm{O}_{4}\right)$ and further developed for the application of electromagnetism. As catalysts, $\mathrm{Fe}_{3} \mathrm{O}_{4}$ is used for removal of $p$-nitrophenol from water. However, since the carbon-supported Pd/Au material system was developed and optimized for catalysis, here, carbon-supported $\mathrm{Fe}_{3} \mathrm{O}_{4}$ NPs were developed for electromagnetic applications. There has been growing interest in tuning the magnetic properties of materials at room temperature with the use of external electric fields, for longterm applications in data storage and spintronic devices. While a complete reversible change of material properties has not yet been achieved, some success in partial switching has been achieved using multiferroic spinel structures such as $\mathrm{Fe}_{3} \mathrm{O}_{4}$. These materials experience a change in magnetic moment at room temperature when exposed to the electric fields generated by electrochemical cells such as lithium ion batteries (LIBs) and supercapacitors (SC). In the past, a $1 \%$ reversible change was observed in $\mathrm{Fe}_{3} \mathrm{O}_{4}$ using LIBs. Here, building on the developments from previous material system, $\mathrm{Fe}_{3} \mathrm{O}_{4} \mathrm{NPs}$ were 
directly hybridized onto the graphene support in order to increase the observable change in magnetic moment. The material was systematically designed and tested for this application, including a study of the material formation. A simple, environmentally friendly synthesis using the solvothermal process was implemented to make the graphene-supported $\mathrm{Fe}_{3} \mathrm{O}_{4}$ NPs. This new material was found to produce a reversible change of up to $18 \%$ in a LIB. In order to overcome some of the difficulties of testing with a LIB, a corresponding hybrid $\mathrm{SC}$ was designed, built and calibrated. The graphene-supported $\mathrm{Fe}_{3} \mathrm{O}_{4} \mathrm{NPs}$ were found to produce a net $2 \%$ reversibility in the SC, which has not been reported before. The results from both the LIB and SC were analyzed to better understand the mechanism of switching in a spinel ferrite such as $\mathrm{Fe}_{3} \mathrm{O}_{4}$, which can help optimize the material for future applications.

The focus of this dissertation was on the development of a methodology for carbonsupported TM and TMO NPs for specific applications. It is envisioned that this approach and strategy will contribute towards the future optimization of similar material systems for a multitude of applications. 


\section{Acknowledgements}

I would like to thank my research advisor, Dr. Jun Jiao, for her guidance and support in my time here at Portland State University. Dr. Jiao has been a role model over the past few years and has always pushed me to reach my highest potential. I'd like to thank my committee members, Dr. Tratnyek, Dr. Meekisho and Dr. Daescu. Thank you to Dr. Tratnyek for helping me develop both academically and professionally. I would also like to thank Dr. Meekisho, for the mentorship, encouragement and kind words over the years. Thank you to Dr. Daescu for stepping in and helping at the last moment.

I would like to thank members of Dr. Jiao’s group, specifically to Andrew, Otto, Lester, Simon and Micah. Thank you to the students I've had the pleasure to work with - Candice, Ryan, Nathan, John, Erin, Zach, Shree and Cass. I'd also like to thank the REU students I've worked with-Arianna, Riley, Elaine, Lydia and Lexi. I am thankful to the Mechanical and Materials Engineering Department; and collaborators Graham, Alvin, Jason, James, Dr. Chen, Dr. Goforth and Dr. Longo.

I will always be grateful to my family — my father for lending me his strength, my sister for teaching me resilience and my husband for always standing by me. Thank you to Ammamma, my sea of aunts, and my in-laws for all the support. This thesis is dedicated to my mother, the bravest and most courageous person I've known. We're both just fools on a hill. I miss you, mom.

Portions of this work was supported in part by NSF awards No. 1507707, No. 1508115 and No. 1560383, Oregon Nanoscience and Microtechnologies Institute (ONAMI) and NIH Build EXITO program. 


\section{Table of Contents}

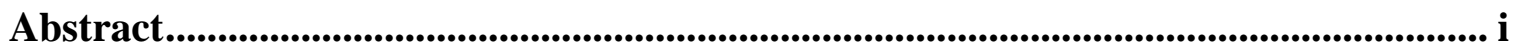

Acknowledgements ....................................................................................................... iv

List of Tables ................................................................................................................ vii

List of Figures............................................................................................................ viii

List of Abbreviations and Symbols ............................................................................... xii

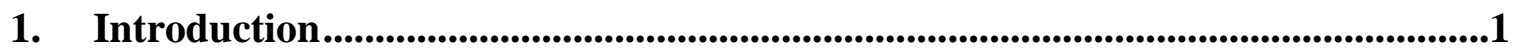

1.1 Background and Applications of Transition Metal Nanoparticles ............................ 1

1.2 Problem Statement and Layout ......................................................................... 3

1.3 Organization of Thesis Based on Experimental Approach....................................... 5

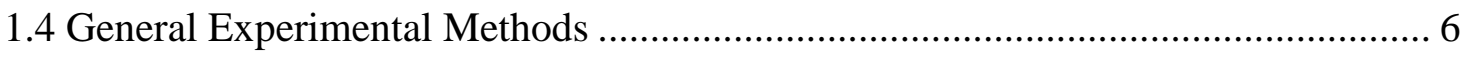

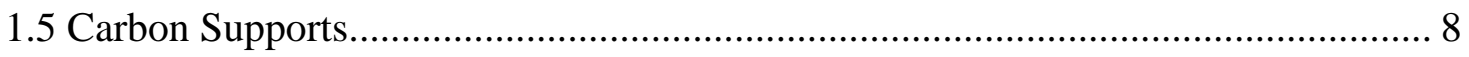

2. Carbon-Supported Pd/Au NPs .....................................................................................10

2.1 Current Literature: Status and Limitations ........................................................ 10

2.2 Design of Catalyst for Removal of Trichloroethylene from Groundwater............. 13

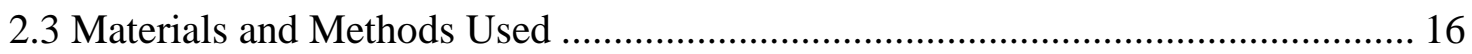

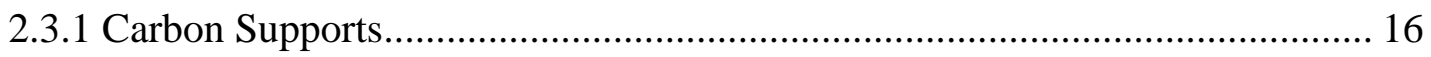

2.3.2 Synthesis of Carbon-Supported Pd/Au Nanoparticles ..................................... 18

2.3.3 Material Characterization.......................................................................... 19

2.3.4 Catalytic Reactivity Testing ..................................................................... 19

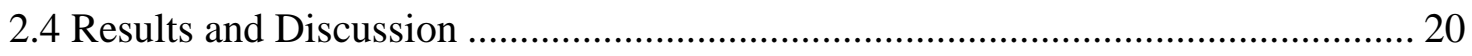

2.4.1 Liquid Exfoliated Graphene.................................................................. 21

2.4.2 Electron Microscopy characterization of Graphene and GAC supported Pd/Au

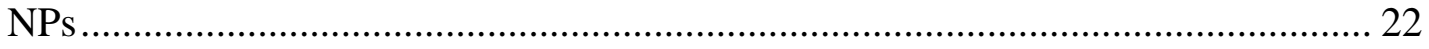

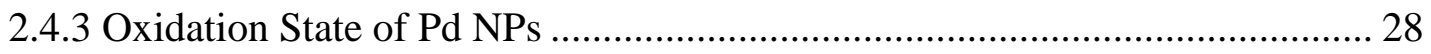

2.4.4 Surface Area Characterization for GAC-based Materials ................................. 31

2.4.5 TCE Removal by Carbon-Supported Pd/Au NPs ............................................ 33

2.4.6 Kinetics and Mechanism for TCE Removal .................................................... 38

2.4.7 Mechanism of Formation of Carbon-Supported Pd/Au NPs ............................ 41

2.4.8 Role of Carbon Support for Pd/Au Nanoparticle-Based Catalysts ................... 56

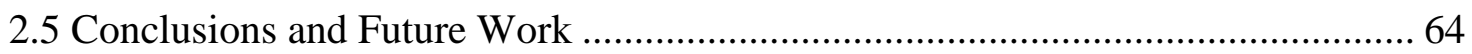




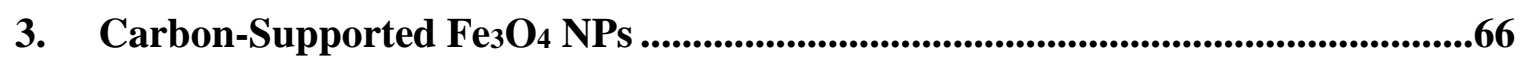

3.1 Current Literature: Status and Limitations …………........................................... 66

3.2 Design of Material and Electrode for Control of Magnetism in an Electric Field . 69

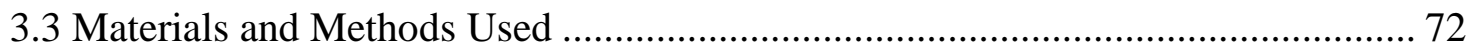

3.3.1 Synthesis of Carbon-Supported $\mathrm{Fe}_{3} \mathrm{O}_{4}$ Nanoparticles..................................... 72

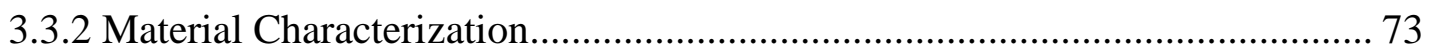

3.3.3 Electrochemical and Magnetic Measurements ................................................. 74

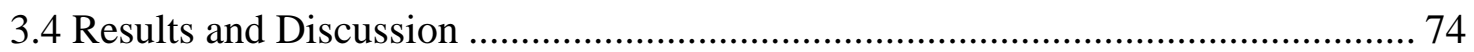

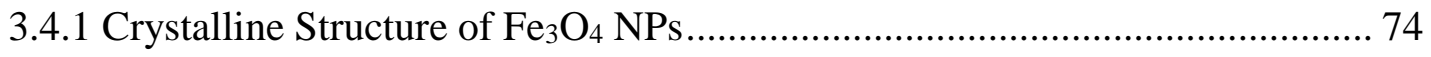

3.4.2 Design of Electrodes to Minimize Binder …………………......................... 77

3.4.3 Control of Magnetic Moment in Electric Fields Generated by LIBs............... 79

3.4.4 Mechanism of Magnetic Switching of Graphene-Supported $\mathrm{Fe}_{3} \mathrm{O}_{4} \mathrm{NPs}$ in LIBs

3.4.5 Mechanism of Magnetic Switching of Graphene-Supported $\mathrm{Fe}_{3} \mathrm{O}_{4}$ NPs in SCs 87

3.4.6 Control of Magnetic Moment in Electric Fields Generated by SCs ................ 90

3.4.5 Construction of Supercapacitor to Measure Change in Magnetic Moment of

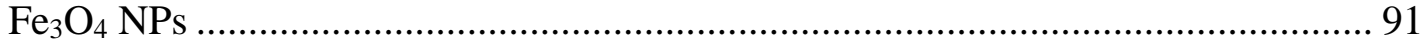

3.4.8 Mechanism of Formation of Graphene-Supported $\mathrm{Fe}_{3} \mathrm{O}_{4}$ NPs ........................ 94

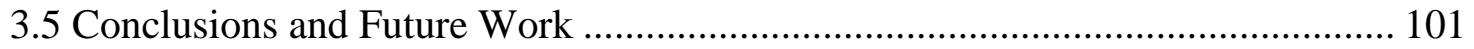

4. Summary and Outlook ............................................................................................102

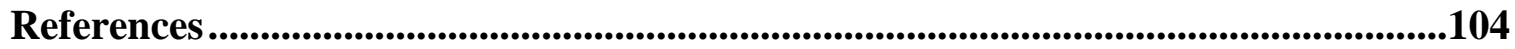




\section{List of Tables}

Table 1: Specific surface area of catalysts calculated using BET analysis ..................... 31

Table 2: Observed and Normalized TCE Decomoposition Rates................................... 41

Table 3. Range and Average size of the NP clusters for the various materials ............... 60 


\section{List of Figures}

Figure 1. Flow diagram summarizing the research activities carried out in this study for (a) carbon-supported Pd/Au NPs, for catalytic applications and (b) carbon-supported $\mathrm{Fe}_{3} \mathrm{O}_{4} \mathrm{NPs}$, for electromagnetic applications.......................................................... 6

Figure 2. Schematic formation of TM and TMO NP during the synthesis process developed in this study

Figure 3. Detailed aspects of the carbon-supported Pd/Au NPs material synthesis, characterization and catalytic testing.

Figure 4. (a) Low magnification TEM of a graphene produced after liquid exfoliation, (b) Raman spectrum collected from sample, marked by the red circle as seen in inset (c).

Figure 5. (a) SEM images with SE detector showing surface of NPs and (b) BSE detector showing contrast of metals making up the NPs. Arrows indicate regions of interest. (c) Schematic representation of $\mathrm{Pd}_{\text {Shell }} \mathrm{Au}_{\text {Core }}$ structure. (d) TEM image of $\mathrm{Pd} / \mathrm{Au}$ NPs on graphene with two types of structures, Structure 1 and Structure 2, marked by boxes. (e) Dark-field STEM image of Structure 2 revealing small NPs dispersed evenly through graphene, EDS spectrum of this in (f) indicates strong Pd peaks but no $\mathrm{Au}$, indicating the NPs are Pd on carbon support; $\mathrm{Cu}$ is from the grid. Structure 1 is examined in Figure 6.23

Figure 6. TEM analysis of Structure 1. (a) TEM image of a cluster with a less dense material surrounding a denser core. HRTEM images of (b) Pd and (c) Au NPs having observable lattice structures and the respective FFT insets show the NPs are crystalline. (d) STEM dark-field image of this cluster confirms two different materials with coreaggregated shell configuration. The arrow points towards an area where the aggregate $\mathrm{Pd}$ shell does not uniformly cover the Au core. EDS maps indicate core is $\mathrm{Au}(\mathrm{e})$, and aggregate shell is $\mathrm{Pd}(\mathrm{f})$ with (g) overlay confirming $\mathrm{Pd}_{\text {Shell }} \mathrm{Au}_{\text {Core }}$ structure.

Figure 7. TEM analysis of (a) a GAC particle with inset (b) showing crystallinity of some smaller, embedded particles. (c) a larger cluster which appears be made of $\mathrm{Pd} / \mathrm{Au}$, according to the EDS spectrum in (d). (e) Dark-field STEM image of the GAC supported sample showing EDS maps of (f) carbon, (g) Pd and (h) Au.

Figure 8. STEM/EELS map and analysis of the bimetallic cluster. (a) STEM image with EELS maps of (b) Pd L, (c) Au M and (d) overlaid maps showing a Pd $\mathrm{Phell}_{\text {Sucore }}$ structure. (e) EELS spectrum from the sample compared with a metallic Pd standard and a $\mathrm{Pd}^{2+}$ standard. Upon comparison, the Pd spectrum from the sample was found to resemble the metallic standard.

Figure 9. Concentration normalized $\left(\mathrm{C} / \mathrm{C}_{0}\right)$ TCE disappearance rates of (a) as-made catalysts, commercial Pd on GAC and plain GAC as control. (b) Significance of SSA of GAC-supported catalysts on TCE disappearance rate. All cases began with $50 \mathrm{ppm}$ of TCE in a hydrogen headspace... 34

Figure 10. TCE disappearance when exposing the catalyst to consecutive doses of 50 ppm of contaminant in a hydrogen headspace.

Figure 11. TCE disappearance with (a) Au only samples and GAC, (b) Disappearance by the catalyst at various molar Pd:Au loading on $70 \mu \mathrm{m}$ GAC, exposed to $50 \mathrm{ppm}$ of TCE in a hydrogen headspace. 
Figure 12. $\mathrm{Pd} / \mathrm{Au}$ NPs on GAC degrading TCE at lower concentrations....................... 37

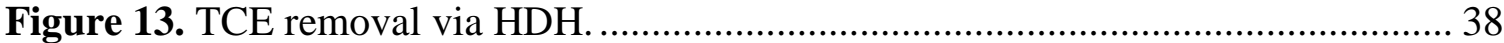

Figure 14. TCE removal rates for Pd/Au catalysts on $800-400 \mu \mathrm{m} \mathrm{GAC}$ and on $70 \mu \mathrm{m}$ GAC as compared to a commercial catalyst. The $k_{M}$ is the degradation rate normalized to the mass concentration of $\mathrm{Pd}$, while the $k_{S A}^{\prime}$ is the rate normalized to the SSA of the catalyst (with units $\mathrm{L} \mathrm{g}_{\mathrm{Cat}} \mathrm{g}_{\mathrm{Pd}}{ }^{-1} \mathrm{~m}^{-2} \mathrm{~min}^{-1}$ ).

Figure 15. TEM images of (a) Pd NPs on graphene at a low magnification and (b) HRTEM image showing crystallinity of NPs. (c) Low magnification of Au NPs showing sparsely scattered NPs over support and (d) HRTEM image showing crystallinity of these NPs. 44

Figure 16. TEM of a sample in which both the Pd and Au precursors were sonicated and allowed to react for the same amount of time, producing non-uniform bimetallic clusters. Images (a), (b) and (c) were taken on different locations of the same sample. Arrows point to areas revealing the smaller Au cores deep inside the thicker Pd aggregate shells.

Figure 17. (a) TEM of a cluster of NPs made using ethanol as the solvent. (b) Dark-field STEM imaging of a cluster in the same cluster. A part of the cluster marked with the square was further examined in (e). EDS Maps (d) and (e) are of Pd and Au respectively. (e) A part of the cluster from (b) shows a less dense material covering a denser material. Extracted EDS spectrums from (f) the center and (g) edge show the Au and Pd peaks in the center and only Pd peaks at the edge, suggesting an Au core surrounded by a uniform Pd shell.

Figure 18. TEM image of a specimen with synthesis time controlled to 1 hour, allowing the observation of structure phenomena of an incomplete reaction. (a) Low magnification image showing different structures present on the same sample; areas marked by boxes, called Structure 1 and Structure 3, have been examined further. (b) Cluster strongly resembling Structure 1, (c) dark-field STEM image of this cluster confirming $\mathrm{Pd}_{\text {Shell }} \mathrm{Au}_{\text {Core }}$ formation. Structure 3 is examined in Figure 19.

Figure 19. Images showing Structure 3. (a) TEM image and corresponding dark-field STEM image in inset (b). (c) A closer look at this cluster. EDS Maps of this cluster for (d) Au and (e) Pd.

Figure 20. SEM images of (a) sample made at room temperature, and (b) at higher magnification, with (c) EDS spectrum showing NPs are made of Pd/Au. (d) sample made at $70^{\circ} \mathrm{C}$, and imaged at higher magnification, (e), with (f) EDS spectrum showing NPs are made of $\mathrm{Pd} / \mathrm{Au}$. The difference in size between the NPs made at the two synthesis temperatures is apparent. 54

Figure 21. SEM images of $\mathrm{Pd} / \mathrm{Au}$ NPs on various carbon supports. From left to right, images increase in magnification. (a - c) Images of GAC-supported Pd/Au NPs, showing (a) dispersion, (b) size, and (c) topography of these NPs. (d-f) are images of carbonblack supported Pd/Au NPs, showing (d) wider distribution of NPs and (e) compactness of the NPs, (f) seen at higher magnifications. $(g-h)$ are images of graphite-Pd/Au NPs showing $(\mathrm{g})$ poor distribution of NPs over the surface of support, and (h) relatively larger size of the NPs and (i) at a higher magnification. Images $(\mathrm{j}-\mathrm{k})$ are of the $\mathrm{Pd} / \mathrm{Au} \mathrm{NPs}$ on graphite nanoplates showing (j) large number of NPs over the surface, $(k)$ relatively 
larger size and (1) the morphology of the NPs. Arrows are provided to help with identification of NP clusters

Figure 22. Retention of (a) Pd NP and (b) Au NP on the GAC, carbon black, graphite and graphite nanoplates supports during synthesis. (c) The decomposition of TCE over time, plotted as normalized concentration of TCE over time, in the presence of just carbon supports and (d) Pd/Au NPs hybridized to the carbon supports.

Figure 23. Adsorption of TCE with GAC received as-is, with heating under vacuum and in air, and finally after an acid bath. 64

Figure 24. Electron spin directions in (a) ferromagnetic, (b) ferrimagnetic and (c) antiferromagnetic materials.

Figure 25. Schematics of (a) conventional electrodes, (b) electrode design used in this project and (c) eventual goal of the project.

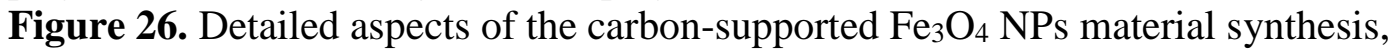
electrode fabrication, electrochemical testing and magnetic testing.

Figure 27. TEM images of $\mathrm{Fe}_{3} \mathrm{O}_{4}$ NPs on graphene at (a) low magnification, (b) high magnification showing lattice fringes and (c) showing an electron diffraction pattern. (d) XRD pattern of these NPs.

Figure 28. TEM images of (a) low magnification of a cluster of commercial $\mathrm{Fe}_{3} \mathrm{O}_{4} \mathrm{NPs}_{\text {. }}$ (b) Higher magnification of these NPs, indicating a large variation in size. Inset (c) is an image from an edge, showing lattice fringes. (d) XRD spectrum of NPs, confirming

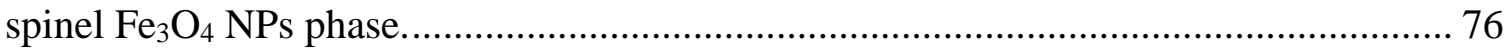

Figure 29. Schematic representation of the electrode designs tested in this project. (a) An electrode made the conventional way, using commercial NPs and carbon black, (b) electrode using commercial NPs but with graphene and (c) the new design of the electrode with NPs directly hybridized onto graphene.

Figure 30. SEM images of the surface of the electrodes at (a) low magnification revealing relatively even surface, (b) higher magnification showing high surface area and (c) highest magnification showing pores where the liquid escaped from the slurry during vacuum drying of the sample. 78

Figure 31. Characteristic curves for LIB with $\mathrm{Fe}_{3} \mathrm{O}_{4} \mathrm{NP}$ hybridized on graphene electrode. (a) CV and (b) charge/discharge curves.

Figure 32. The in-situ magnetic measurements collected during electrochemical discharging/charging of LIB with (a) commercial $\mathrm{Fe}_{3} \mathrm{O}_{4} \mathrm{NPs}$, graphene and PVDF, and (b) graphene supported $\mathrm{Fe}_{3} \mathrm{O}_{4} \mathrm{NPs}$ and PVDF. The saturation magnetization of $\mathrm{Fe}_{3} \mathrm{O}_{4}$ NPs reversibly changes up to $2 \%$ and $18 \%$ respectively for each specimen, as a function of voltage.

Figure 33. Ex-situ measurement of magnetic moment of the LIB with commercial $\mathrm{Fe}_{3} \mathrm{O}_{4}$ NPs, graphene and PVDF in the (a) original state with inset showing complete data and (b) after charge, with the inset showing total cycle data. The increase in thickness of hysteresis curve indicates a change in magnetic moment.

Figure 34. 3D representation of the crystal structure of $\mathrm{Fe}_{3} \mathrm{O}_{4}$ (a) showing locations of cation $\mathrm{A}\left(\mathrm{Fe}^{2+}\right.$ ions) in octahedral sites and cation $\mathrm{B}\left(\mathrm{Fe}^{3+}\right.$ ions) split across the remaining octahedral and tetrahedral sites; (b) showing the magnetic moments of the ions, and the net magnetic moment. 
Figure 35. Superexchange interactions between the $\mathrm{Fe}^{3+}$ and $\mathrm{Fe}^{2+}$ cations and the $\mathrm{O}^{2-}$ anion, (a) when the angle between the ions is $90^{\circ}$ usually leading to ferromagnetism and (b) when the angle between the ions is $120^{\circ}$ usually leading to antiferromagnetism...... 85

Figure 36. The CV of a LIB showing damage to material, indicated by the disappearing peaks. Arrows point to peaks of first cycle.

Figure 37. Construction of SC. (a) working electrode with graphene supported $\mathrm{Fe}_{3} \mathrm{O}_{4}$ NPs, (b) carbon counter, (c) the assembled SC and (d) the clamp system used to test the $\mathrm{SC}$.

Figure 38. Characteristic curves for $\mathrm{SC}$ with $\mathrm{Fe}_{3} \mathrm{O}_{4} \mathrm{NP}$ hybridized on graphene electrode. (a) CV showing redox peaks, as indicated by the arrows, with inset showing EDLC layer behavior of SC, and (b) charge/discharge curves.

Figure 39. (a)Change in magnetic moment as a function of voltage for $\mathrm{Fe}_{3} \mathrm{O}_{4} \mathrm{NPs}$ hybridized on graphene as tested in a SC.(b) Schematic explanation for the change in magnetic moment for this SC.

Figure 40. Different configurations of the SC with corresponding CVs. (a) threeelectrode carbon-carbon SC, (b) two-electrode carbon-carbon SC, (c) three-electrode carbon-carbon SC with potassium ferrocyanide to identify redox peaks of $\mathrm{Fe}^{2+} / \mathrm{Fe}^{3+}$ and (d) two-electrode $\mathrm{Cu}-\mathrm{Cu} \mathrm{SC}$.

Figure 41. (a) Test of cyclability of the electrodes, plotting capacitance retained over cycle number. (b) TEM images of synthesized $\mathrm{Fe}_{3} \mathrm{O}_{4}$ NPs after 1000 cycles, with inset (c) showing crystallinity is retained by the NPs after this long cycling....

Figure 42. Raman spectra of iron oxide formed via solvothermal synthesis in ethanol at different synthesis times.

Figure 43. TEM images of graphene supported iron oxide NPs after $(\mathrm{a}-\mathrm{c}) 2$ hours and $(b-f) 48$ hours of synthesis time. (a) low magnification image of the NPs on graphene after just 2 hours of synthesis, (b) relatively higher magnification showing spread of NPs on the support, and (c) a closer look at a NPs with inset FFT revealing its crystalline nature. (d) low magnification image of NPs after 48 hours, (e) relatively higher magnification showing angular shape of NPs and (f) shows the lattice fringes and inset of FFT indicating the NPs are also crystalline.

Figure 44. (a) TEM image of $\mathrm{Fe}_{3} \mathrm{O}_{4}$ formed at $220^{\circ} \mathrm{C}$ showing some particles with hexagonal shape. Arrows point towards some NPs where the shape is distinct. (b) HRTEM of a NP and inset FFT. (c) Raman spectra of the materials synthesized at $220^{\circ} \mathrm{C}$, $180^{\circ} \mathrm{C}$ and $160^{\circ} \mathrm{C}$.

Figure 45. $(\mathrm{a}-\mathrm{b})$ Characterization of specimen made using acetone at $180^{\circ} \mathrm{C}$ (a) TEM image of the specimen after 24 hours showing formation of some quasi-cubic NPs, arrows point at prominent NPs. (b) low magnification image showing these cubic NPs are uniformly present in the specimen. (c) HRTEM image with FFT inset showing these NPs are crystalline in nature. (d) Raman spectra of the materials made at different times up to 24 hours. $(\mathrm{e}-\mathrm{g})$ Characterization of specimen made using acetone at $220^{\circ} \mathrm{C}$. (e) TEM image of specimen at $220^{\circ} \mathrm{C}$ after 24 hours showing mostly quasi-cubic NPs. (f) HRTEM image with inset suggesting these NPs are also crystalline. (g) Raman spectra showing at $220^{\circ} \mathrm{C}, 180^{\circ} \mathrm{C}$ and $160^{\circ} \mathrm{C}$ with acetone, $\alpha-\mathrm{Fe}_{2} \mathrm{O}_{3}$ phase NPs are formed. 


\section{List of Abbreviations and Symbols Abbreviations}

1,1-DCE
AGM
Alumina
Au
BET
BSE
CERCLA
cis-DCE/cis-1,2-DCE
Cl
CT
Cu
CV
DI Water
ECD
EDLC
EDS
EELS
EPA
FFT
FID
GAC
GC
GC-MS
H2
HDH
HRTEM
Hydrotalcite
Ir
LIB
LSMO
Magnetite
ME
min
Ni
NMP
NPs
O
Pd
ppb
ppm
Pt

1,1-dichloroethene

alternating grating gradient magnetometer

$\mathrm{Al}_{2} \mathrm{O}_{3}$

gold

Brunauer-Emmett-Teller

back scattered electron

Comprehensive Environmental Response and

Compensation Liability Act of 2017

cis-1,2-dichloroethene

chlorine

carbon tetrachloride

copper

cyclic voltammetry

deionized water

electron capture detector

electrostatic double-layer capacitance

energy dispersive X-ray spectroscopy

electron energy loss spectroscopy

Environmental Protection Agency

Fast Fourier Transform

flame ionization detector

granular activated carbon

gas chromatography

gas chromatography-mass spectrometer

hydrogen molecule

hydrodehalogenation

high resolution transmission electron microscopy

$\left(\mathrm{Mg}_{6} \mathrm{Al}_{2} \mathrm{CO}_{3}(\mathrm{OH})_{16} \cdot 4\left(\mathrm{H}_{2} \mathrm{O}\right)\right.$

iridium

lithium ion battery

$\mathrm{La}_{0.74} \mathrm{Sr}_{0.26} \mathrm{MnO}_{3}$

$\mathrm{Fe}_{3} \mathrm{O}_{4}$

magnetoelectric effect

minutes

nickel

n-methyl-2-pyrrolidone

nanoparticles

oxygen

palladium

parts per billion

parts per million

platinum

xii 


$\begin{array}{ll}\text { PVDF } & \text { polyvinylidene fluoride } \\ \text { Rh } & \text { rhodium } \\ \text { Ru } & \text { ruthenium } \\ \text { SC } & \text { supercapacitor } \\ \text { SE } & \text { secondary electron } \\ \text { SEM } & \text { scanning electron microscopy } \\ \text { Si } & \text { silicon } \\ \text { Silica } & \text { SiO } 2 \\ \text { SQUID } & \text { superconducting quantum interference device } \\ \text { SSA } & \text { specific surface area } \\ \text { STEM } & \text { scanning transmission electron microscopy } \\ \text { TCE } & \text { trichloroethene, trichloroethylene } \\ \text { TCP } & \text { trichloropropane } \\ \text { TEM } & \text { transmission electron microscopy } \\ \text { Titania } & \text { TiO } 2 \\ \text { TM } & \text { transition metal } \\ \text { TMO } & \text { transition metal oxide } \\ \text { trans-DCE/trans-1,2-DCE } & \text { trans-1,2-dichloroethene } \\ \text { VC } & \text { vinyl chloride } \\ \text { VOCs } & \text { volatile organic compounds } \\ \text { VSM } & \text { Vibrating sample magnometer } \\ \text { WHO } & \text { World Health Organization } \\ \text { XRD } & \text { X-ray diffraction } \\ \text { Zn } & \text { zinc }\end{array}$

\section{Symbols}

\begin{tabular}{|c|c|}
\hline$a_{s}$ & specific surface area of Pd NPs $\left(\mathrm{m}^{2} / \mathrm{g}_{\mathrm{Pd}}\right)$ \\
\hline$a_{s}^{\prime}$ & specific surface area of catalyst $\left(\mathrm{m}^{2} / \mathrm{g}_{\mathrm{Cat}}\right)$ \\
\hline$C$ & initial mass concentration of TCE in the bulk fluid ( $\left.\mathrm{g}_{\mathrm{TCE}} / \mathrm{L}\right)$ \\
\hline$C / C_{0}$ & mass concentration of TCE normalized to initial mass concentration \\
\hline$C_{P d}$ & mass concentration of $\mathrm{Pd}$ in each sample per reaction volume $\left(\mathrm{g}_{\mathrm{Pd}} / \mathrm{L}\right)$ \\
\hline$C_{T C E}$ & mass concentration of TCE in the bulk fluid $\left(\mathrm{g}_{\mathrm{TCE}} / \mathrm{L}\right)$ \\
\hline$k_{o b s}$ & observed pseudo first-order rate constant $\left(\mathrm{min}^{-1}\right)$ \\
\hline$k_{o b s, A v g}$ & average value of $k_{o b s}\left(\mathrm{~min}^{-1}\right)$ \\
\hline$k_{M}$ & rate constant normalized for mass $\left(\mathrm{L} \mathrm{g}_{\mathrm{Pd}}{ }^{-1} \mathrm{~min}^{-1}\right)$ \\
\hline$k_{M, A v g}$ & average value of $k_{M s}\left(\mathrm{~L} \mathrm{~g}_{\mathrm{Pd}}^{-1} \min ^{-1}\right)$ \\
\hline$k_{S A}$ & rate constant normalized for surface area of $\mathrm{Pd}\left(\mathrm{L} \mathrm{m}^{-2} \mathrm{~min}^{-1}\right)$ \\
\hline$k_{S A}^{\prime}$ & $\begin{array}{l}\text { mass-normalized rate constant, normalized to specific surface area } \\
\text { of catalyst }\left(\mathrm{L} \mathrm{g}_{\mathrm{Cat}} \mathrm{g}_{\mathrm{Pd}}^{-1} \mathrm{~m}^{-2} \mathrm{~min}^{-1}\right)\end{array}$ \\
\hline$\kappa_{S}$ & average value of $k_{S A}^{\prime}\left(\mathrm{L}_{\mathrm{Cat}} \mathrm{g}_{\mathrm{Pd}}^{-1} \mathrm{~m}^{-2} \mathrm{~min}^{-1}\right)$ \\
\hline & time (min) \\
\hline
\end{tabular}




\section{Introduction}

The following thesis presents a scientific investigation into the design, synthesis and characterization of transition metal nanoparticles, for catalytic and electromagnetic applications. As this work was done for fulfillment of a degree in Mechanical Engineering with emphasis on Material Science, it is particularly aimed at gaining a better understanding of, and manipulating, material properties with specific, practical applications in catalysis and electromagnetism. The focus of this research was on the synthesis and characterization of two distinct material systems: carbon-supported transition metal (TM) nanoparticles (NPs), specifically pallidum (Pd) and gold (Au) NPs, for catalytic applications; and carbon-supported transition metal oxide (TMO) NPs, specifically magnetite $\left(\mathrm{Fe}_{3} \mathrm{O}_{4}\right)$ NPs for electromagnetic applications. First, the carbonsupported Pd/Au NPs material was developed using an environmentally friendly synthesis and design, and this methodology was applied to develop the carbon-supported $\mathrm{Fe}_{3} \mathrm{O}_{4} \mathrm{NPs}$ material. Both material systems were developed for specific applications, and hence while the details of synthesis and testing for each material system is different, the way the materials were designed and methodology in development of the systems is similar. Thus, the layout in this thesis starts with a common introduction and followed by a detailed discussion of the particulars of each material system in individual sections.

\subsection{Background and Applications of Transition Metal Nanoparticles}

TMs are elements whose atoms have partially filled $d$ sub-shell, or which can produce cation with an incomplete $d$ sub-shell. This definition generally applies to the $d$-block of elements in the periodic table, groups 3 to 12 . TMs are used for a variety of applications 
including construction, manufacturing, pipes, wire production etc. TMs often form compounds in many oxidation states, due to the relatively low energy gap between the different possible oxidation states. This promotes several remarkable properties, for instance serving as excellent catalysts for various important reactions and exhibiting magnetic properties due to the presence of unpaired $d$ electrons. TM and TMO NPs have several applications in synthesis of pigments[1], drug delivery[2], catalysis[3], and electronic and magnetic materials[4]; in this study, the focus was on the catalytic properties of $\mathrm{Pd} / \mathrm{Au}$ NPs and the electromagnetic properties of $\mathrm{Fe}_{3} \mathrm{O}_{4} \mathrm{NPs}$.

For catalytic applications, the emphasis of this research was on removal of volatile organic compounds (VOCs) from groundwater. Trichloroethylene (TCE) was chosen as the model contaminant, for which a TM-based catalyst was designed and tested. The degradation of TCE usually occurs via hydrodehalogenation (HDH), in the presence of TM-based catalysts such as Pd, platinum (Pt), iridium (Ir), rhodium (Rh), copper $(\mathrm{Cu})$, zinc $(\mathrm{Zn})$, ruthenium $(\mathrm{Ru})$ and nickel $(\mathrm{Ni})$. In particular, Pd-based catalysts are more active, stable and selective for desired end-products, and are often less toxic [5]. Additionally, Au has shown to be a good promotor of Pd for $\mathrm{HDH}$. For these reasons, among the available catalytically-active TMs, Pd and Au NPs are the focus of this research for TCE removal. This catalyst was optimized for removal of TCE, however it can also be used to remove similar chlorinated VOCs such as trichloropropane (TCP) and carbon tetrachloride (CT).

In addition to Pd, magnetite has been shown to be an effective catalyst for the removal of $p$-nitrophenol from water [6]. Here, as the focus for catalytic applications was on $\mathrm{Pd} / \mathrm{Au}$, $\mathrm{Fe}_{3} \mathrm{O}_{4}$ was instead developed for electromagnetic applications, where magnetic moment of 
the material was reversibly controlled using external electric fields. For most electromagnetic applications, controlling magnetic properties by external means without changing temperatures is highly desirable. Multiferroic materials such as $\mathrm{Fe}_{3} \mathrm{O}_{4}$, are often of interest for this switching, specifically spinel ferrites such as $\mathrm{Fe}_{3} \mathrm{O}_{4}, \gamma-\mathrm{Fe}_{2} \mathrm{O}_{3}, \mathrm{MnFe}_{2} \mathrm{O}_{4}$, $\mathrm{ZnFe}_{2} \mathrm{O}_{4}$ and $\mathrm{CuFe}_{2} \mathrm{O}_{4}$ [7]-[10]. For these materials, the mangetoelectric (ME) effect plays a large role in the tunability of magnetic properties. Building on previously published results, here, graphene-supported $\mathrm{Fe}_{3} \mathrm{O}_{4}$ was developed and optimized for an increased in change of magnetic moment in electric fields. This on/off switching of magnetic properties has significant long-term applications in the fields of data storage and spintronics [11].

\subsection{Problem Statement and Layout}

The aim of this research was towards development of a new methodology in which robust, stable and highly versatile carbon-supported TM and TMO NPs can be fabricated, using an environmental-friendly synthesis, for optimization of a material towards a specific application.

The methodology was first developed for the carbon-supported Pd/Au NPs. Treatment of groundwater for VOCs has been a persisting issue over decades. While there is a wealth of information available on the several approaches that have already been taken towards removal of TCE degradation, these are yet to be implemented on a large-scale. Pd-based catalysts offer the most promising solution for the removal of VOCs from water, and have been under study for removal of VOCs over several decades, but often require challenging or expensive synthesis processes for the fabrication of the catalyst material [5]. Thus, the research presented was focused on developing a practical, green synthesis to produce the 
most potent catalyst for TCE removal. In this catalyst, Au was used as a promoter increase catalytic activity and the material was studied both for mechanism of formation and performance against TCE towards optimization. In the process, several parameters such as loading density, support type, synthesis temperature, synthesis time, solvent type and TCE concentration was varied to better understand and manipulate the materials. The results from these variations are shared here in this thesis. A robust and systematic investigation of most of the relevant parameters was carried out for this application. The results show the carbon-supported $\mathrm{Pd} / \mathrm{Au}$ NPs catalysts made using an environmentally process were able to produce double the rate of TCE degradation when compared to commercial Pdbased catalysts. In addition, by varying parameters such as type of carbon support, surface area of carbon support and amount of Au used, the material can be adjusted for cases, where trade-offs can be made between catalyst activity and cost of production.

This methodology of using a green chemistry synthesis to make carbon-supported TM NPs was extended to the development of carbon-supported TMO NPs using a similar environmentally friendly process. A new multiferroic material of graphene-supported $\mathrm{Fe}_{3} \mathrm{O}_{4}$ NPs was specifically designed to optimize response of the control of magnetism in electric fields. The reversible control of magnetism was achieved in $\mathrm{Fe}_{3} \mathrm{O}_{4} \mathrm{NPs}$ with lithium ion battery (LIB) in the past, showing up to a $1 \%$ change [10]. While this result is novel and has not been reported in the past, here this material was manipulated and modify in order to observe a larger magnitude of reversible change of magnetic properties of the $\mathrm{Fe}_{3} \mathrm{O}_{4}$ NPs. In addition, to eliminate some of the shortcomings of a LIB, this material was tested in the electric fields of a supercapacitor (SC), which has not been reported before. 
Here, time has been spent in the design, fabricating and tuning the system to increase $\%$ change of this response in a LIB and, in addition, a matching SC was designed and developed for testing of magnetic response of the $\mathrm{Fe}_{3} \mathrm{O}_{4}$ NPs. Factors like choice of material, structure and properties of the material, synthesis parameters and choice of testing components were systematically manipulated in order to significantly optimize the response. The electrical and magnetic testing results are presented here, showing successful increase in change in magnetic moment to $18 \%$ for $\mathrm{Fe}_{3} \mathrm{O}_{4}$ using a LIB and a $2 \%$ reversible change SC.

\subsection{Organization of Thesis Based on Experimental Approach}

This thesis has been organized into two parts - the first part deals with carbonsupported $\mathrm{Pd} / \mathrm{Au} \mathrm{NPs}$, while the second part deals with carbon-supported $\mathrm{Fe}_{3} \mathrm{O}_{4} \mathrm{NPs}$. As described in Section 1, the carbon-supported Pd/Au NPs material system was developed initially, and this methodology was refined for the carbon-supported $\mathrm{Fe}_{3} \mathrm{O}_{4} \mathrm{NPs}$ system. For the sake of continuity, each section has its own literature review, materials and method, results and conclusions.

While there are differences in the materials and applications discussed in this study, the same systematic approach was taken towards the investigation of material properties and effects. The materials were made using similar eco-friendly synthesis procedures and optimized further, with an aim towards specific applications. Using electron microscopy and other spectroscopy tools, the materials were thoroughly investigated. Each material was tested in their respective systems in a meticulous manner. A rigorous feedback system was used on the synthesis process, hence material properties were optimized for the 
individual applications. This has been elucidated in Figure 1, which lists the major research activities for each research project. Figure 1 (a) shows the step-by-step activities for the project dealing with effective TCE removal, and Figure 1 (b) shows the activities for effective control of magnetism in electric field.
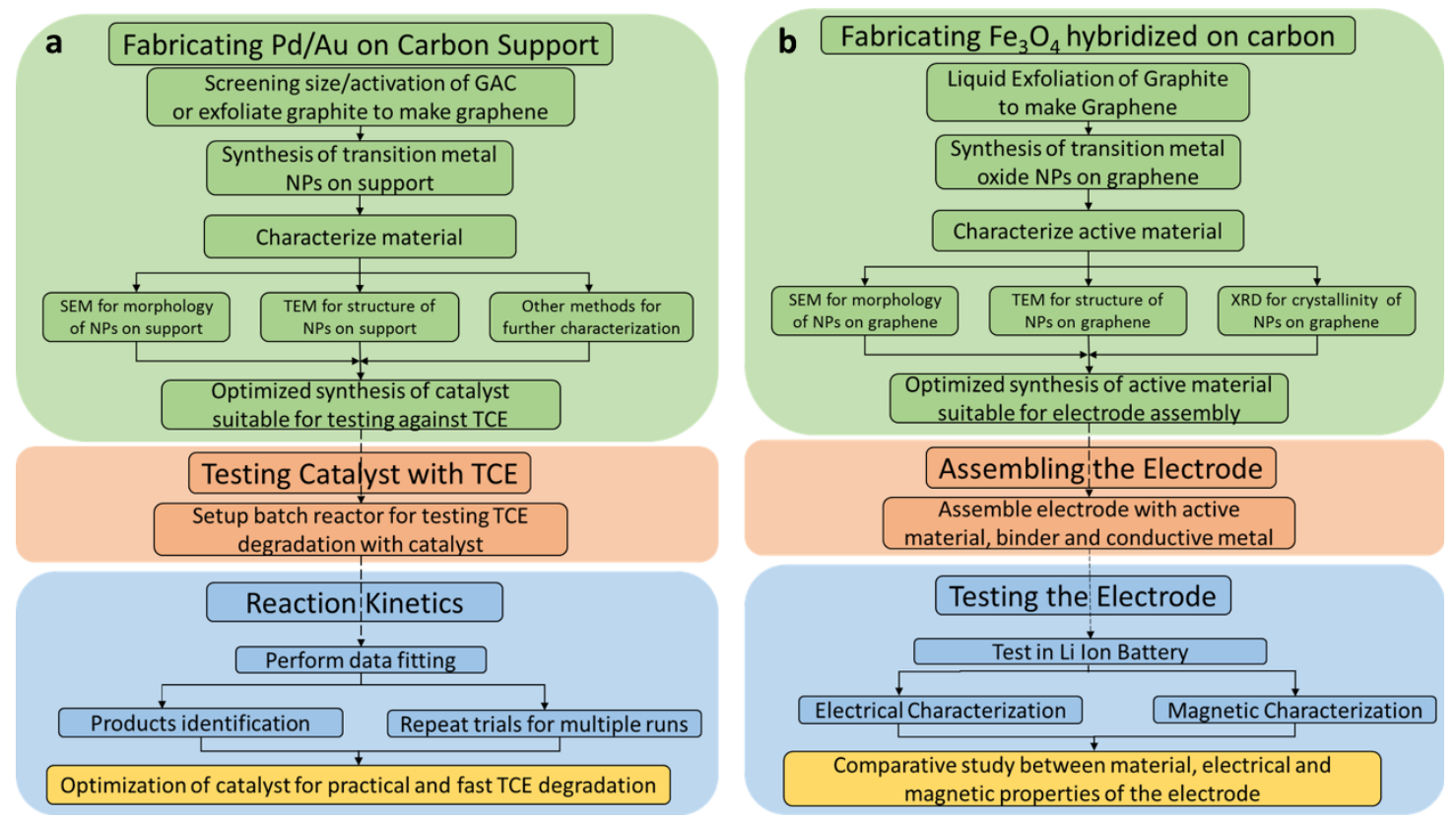

Figure 1. Flow diagram summarizing the research activities carried out in this study for (a) carbon-supported $\mathrm{Pd} / \mathrm{Au} \mathrm{NPs}$, for catalytic applications and (b) carbon-supported $\mathrm{Fe}_{3} \mathrm{O}_{4} \mathrm{NPs}$, for electromagnetic applications.

\subsection{General Experimental Methods}

While the specific materials used for each material system have been described in detail in the respective sections, here a broad overview of the methods section is stated. Of the vast number of ways available to synthesize TM and TMO NPs, a bottom-up method was chosen to synthesize both the TM and TMO NPs in this study. This synthesis process has been illustrated in Figure 2. 


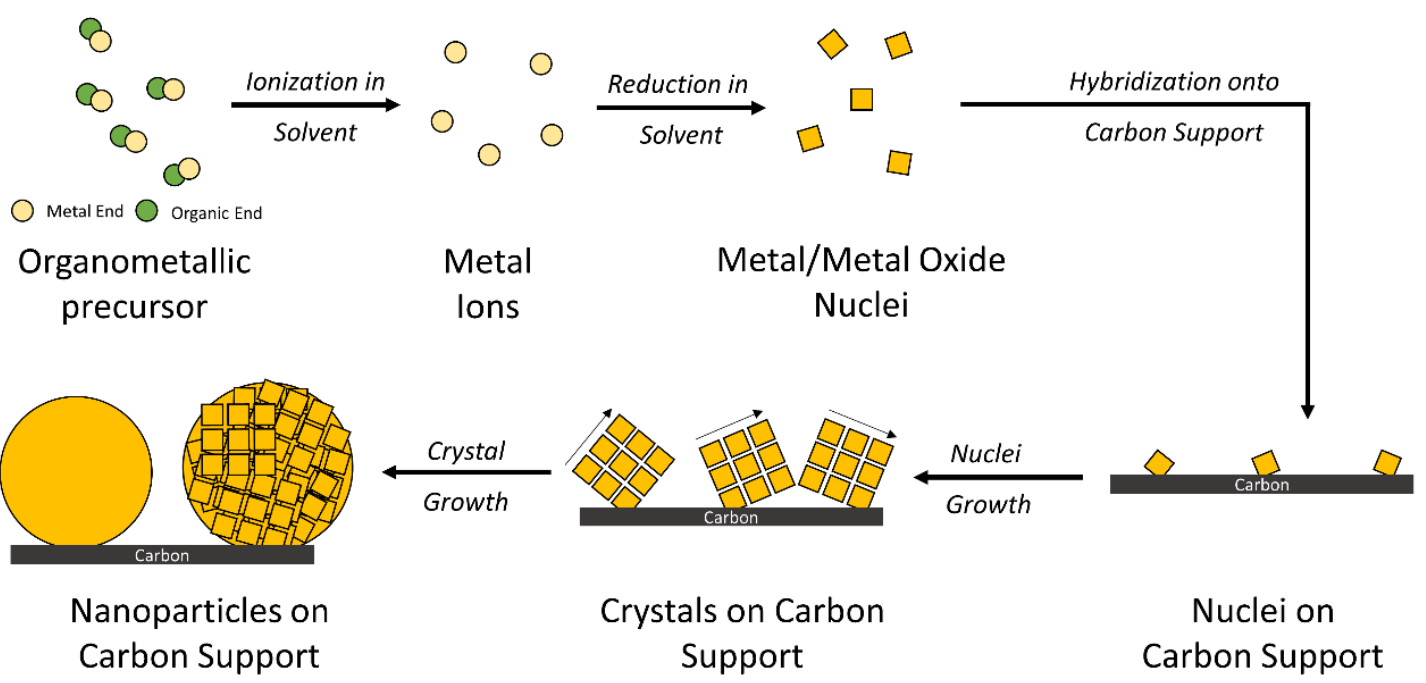

Figure 2. Schematic formation of TM and TMO NP during the synthesis process developed in this study.

Organometallic precursors were used as sources of the metals. This synthesis process was designed such that these precursors used would ionize into metal ions when mixed dissolved in the solvent. When the reaction is initiated with external energy either in the form of soundwaves (sonication) or heat (thermal), these metal ions are reduced to the respective TM or TMO nuclei. In the presence of the carbon support, the nuclei hydride with the surface, anchoring in place. Here, the term "hybridize" refers to the interaction between the metal atoms and carbon support as analyzed by previous collaborators who have simulated this system using DFT calculations [12]. Given enough time to react, these nucleate, crystallize, grow and then agglomerate into NPs. The solution containing solvent and metallic precursor has been referred to as "forming solution", as it used to form the NPs. The effects of some important synthesis variables such as time, temperature and solvent on each material system were studied and are detailed in the respective results section of this thesis. 
This process is a simple, two-step process involving the uniform mixing following by interaction with interaction with solvent. No harsh chemicals, strong redox reagents, surfactants, stabilizers or extreme synthesis parameters were used. Several green chemistry principles were incorporated including design of safer chemicals \& products, using safer solvents \& reaction conditions and using low environmental impact chemicals.

The general characterization was conducted using scanning electron microscope (SEM), transmission electron microscope (TEM), scanning transmission electron microscopy (STEM) and energy dispersive X-ray spectroscopy (EDS). As the testing was specific to application, the tools and instruments used have been described in the respective sections.

\subsection{Carbon Supports}

Supports offer several advantages over individual or bimetallic particles as they enable even particle distribution, prevent particle aggregation, and increase the ease of handling the material for practical use. For this reason, all the NPs in this study were designed to be anchored onto a support.

Carbon is commonly chosen as a support due to its large specific surface area, excellent electron conductivity, and relative chemical inertness. As a support for NPs, it has been found to have better hydrothermal stability when compared to oxide supports such as titania $\left(\mathrm{TiO}_{2}\right)$, alumina $\left(\gamma-\mathrm{Al}_{2} \mathrm{O}_{3}\right)$, mesoporous silica $\left(\mathrm{SiO}_{2}\right)$, and hydrotalcite $\left(\mathrm{Mg}_{6} \mathrm{Al}_{2} \mathrm{CO}_{3}(\mathrm{OH})_{16} \cdot 4\left(\mathrm{H}_{2} \mathrm{O}\right)\right.$ [13]. Additionally, most carbon supports can be easily hybridized with metallic nanoparticles (NPs) [13]-[17]. 
In this study, several types of carbon supports were used. Of these, graphene flakes were used as the main support for both $\mathrm{Pd} / \mathrm{Au}$ NPs and $\mathrm{Fe}_{3} \mathrm{O}_{4}$ NPs. Here, the term "graphene flakes" refers to few-layered graphene as produced using the liquid exfoliation process. Since this quality of graphene is sufficient for the applications of the study, the terms "graphene flakes" and "graphene" have been used interchangeably. More information about this material can be found in Section 2.3 and Section 2.4.1. In addition, granular activated carbon (GAC), graphite and graphite nanoplates were used to support $\mathrm{Pd} / \mathrm{Au}$ NPs. "Graphite nanoplates" refers to thicker, many-layered graphene, as described in Section 2.4.8. 


\section{Carbon-Supported Pd/Au NPs}

In this section, the systematic design, synthesis, characterization, catalytic testing and some optimization of the carbon-supported Pd/Au NPs are discussed. Some of the results presented here have been published previously [17]-[21], and, at the time of writing this thesis, others are under review for publication.

\subsection{Current Literature: Status and Limitations}

More than 50\% of the people in United States use groundwater for drinking and other household uses. Groundwater is used by about $75 \%$ of cities, majority of rural areas and by many factories. The largest use of groundwater is for the irrigation of crops [22],[23]. The groundwater quality is affected by industrial discharges, urban activities, agriculture, groundwater pumpage, and disposal of waste. Contaminants can be from several sources including leaking fuel tanks, or toxic chemical spills, pesticides and fertilizers. In some cases, a well could have been placed in land that was once used for a garbage or chemical dump site. In all these situations and more, chemicals, bacteria and biological agents can be introduced into groundwater as contaminants. These are usually of three types: inorganic, organic and microbial contaminants [24].

Of these contaminants, the focus of this research is in removal of organic contaminants, specifically the VOCs from groundwater. VOCs are used to make plastics, dyes, rubbers, polishes, solvents, crude oil, insecticides, inks, varnishes, paints, disinfectants, gasoline products, pharmaceuticals, preservatives, spot removers, paint removers, degreasers, and many more. When not properly disposed, VOCs often end up in the environment and will persist until externally removed. Exposure to VOCs has several potential health risks and 
other effects, including causing cancer, liver damage, anemia, gastrointestinal disorder, skin irritation, blurred vision, exhaustion, weight loss, damage to the nervous system, and respiratory tract irritation [24].

Chlorinated ethenes, which are a type of VOCs, are a considered to be some of the most harmful of groundwater contaminants [25]. TCE, a well-known VOC was chosen as the model contaminant for this research. It is the most frequently found VOCs in groundwater in the U.S. and often in the highest concentrations [26], while ranking $16^{\text {th }}$ on the Comprehensive Environmental Response and Compensation Liability Act of 2017 (CERCLA) priority list of hazardous compounds [27]. This problem of TCE contamination is global, faced internationally by countries such as Canada, China and other countries [26]. A recent U.S. Geological Survey found that $5 \%$ of ground water sources within the country, which are known to be isolated from contaminant zones, have been detected with TCE, with some sites detected with concentrations in excess of $200 \mathrm{ppb}(1 \mathrm{ppb}=1 \mu \mathrm{g} / \mathrm{L}=0.0076$ $\mu \mathrm{M})$ [25]. For comparison, the World Health Organization (WHO) recommends a drinking water limit of $20 \mathrm{ppb}$ for TCE [26], while the U.S. Environmental Protection Agency (EPA) limits a maximum contaminant level of $5 \mathrm{ppb}$ [28]. Used as a degreasing agent and in textile manufacture, TCE is a known carcinogenic which is highly persistent in groundwater, due to its continual release as a contaminant plume from subsurface deposits and its resistance to biotic and abiotic degradation mechanisms [25], [26].

TCE-contaminated groundwater is conventionally treated using a combination of airstripping and carbon adsorption methods, which displace TCE from water. GAC is the most commonly used material for adsorption of TCE, due to its abundance and inexpensive 
cost. The biggest drawback of using this existing technology is that while TCE is removed from water by GAC, it is not broken down, and so when processing spent (or saturated) GAC, these is a risk associated with re-contamination of the site and/or others. For this reason, alternative methods are sought, which not only remove TCE but also degrade it to a safer form [29].

Recently, chemical treatment processes have gained attention, in which Pd-based materials are used to catalyze aqueous TCE removal. Using these catalysts allows dechlorinate TCE into safer compounds at a faster rate compared to bioremediation, and the reactions are carried out under ambient conditions [29]. In general, Pd-based catalysts tend to be more catalytically active, chemically stable and reaction selective an $\mathrm{Ir}, \mathrm{Rh}, \mathrm{Zn}$ etc. In the presence of $\mathrm{Pd}$, the hydrogen molecule $\left(\mathrm{H}_{2}\right)$ readily dissociates, forming atomic or nascent $\mathrm{H}$ which replace the halogens of a chlorinated ethene. Pd offers several potential advantages including the ability to treat a wide range of pollutants, rapid reaction rates, transformation of contaminants to relatively benign compounds, and applicability in deep aquifers and at high contaminant concentrations where other treatment technologies might be impractical. In fact, field implementations of this technology have confirmed the ability of Pd catalysts to successfully treat halogenated hydrocarbons in groundwater for periods of years [30]. Au was found to be an excellent promotor of Pd, in that it increases the dissociative adsorption of $\mathrm{H}_{2}$ to $\mathrm{Pd}$. For this reason, $\mathrm{Pd}$ is often accompanied with $\mathrm{Au}$ for HDH of VOCs [5].

The synthesis of Pd-based catalysts involve the use of high temperatures, pressures [31] or vacuum [32], [33], toxic chemicals such as sodium borohydride [34], hydrazine[35], 
hydrogen peroxide [36], a pH control with alkaline or acidic components [36], [37] — and some even require the use of surfactants and stabilizers [38]. While these NPs are sometimes fabricated without supports, supports offer several advantages over individual or bimetallic particles. In general, they enable even particle distribution, prevent particle aggregation, and increase the ease of handling the product for practical use. Of the available supports, carbon-based supports are preferred due to their affordability and chemical stability.

\subsection{Design of Catalyst for Removal of Trichloroethylene from Groundwater}

Here, the Pd and Au NPs were combined with carbon supports such as GAC to make a powerful catalyst for the sustainable removal of VOCs from groundwater. As discussed in the previous section, Pd and Au NPs are a natural choice due to their high activity in decomposition of TCE. The chemical potency of these NPs were synergistically combined with the highly adsorptive nature of GAC to yield a very effective catalyst for TCE removal. GAC, through adsorption, removes TCE at a very slow rate. With the addition of the Pd/Au NPs, the removal of TCE is significantly faster, due the combined effects. That is, the adsorption of GAC attracts the TCE towards the NPs-rich surface, and these NPs catalyze the breakdown of TCE into harmless by-products.

In this study, the experimental investigation is focused on a simple green-chemistry approach to produce catalysts made of Pd and Au NPs on carbon supports. Here, the bimetallic Pd/Au NPs were made using a combination of sonochemical and solvothermal processes. These processes were chosen as they do not need an outside reducing agent and does not use harsh chemicals, surfactants, or stabilizers. Additionally, the precursors for 
making the NPs were chosen to have minimal impact on the environment while still being able to form effective catalysts. The process is simple, and since no other chemicals are involved in the synthesis, the chance of impurities affecting the catalyst activity is reduced.

Sonochemical process, or sonication, uses ultrasound waves that pass through water to cause acoustic cavitation (formation, growth and violet collapse of small bubbles) [39]. This cavitation leads to the formation of hydrogen and hydroxyl radicals, which can diffuse into the bulk solution and cause further reactions [40], [41]. When metallic precursors are sonicated, these radicals reduce the metal ion into zero-valent metal particles. In fact, the sonochemistry of specific metal compounds such as $\mathrm{Fe}(\mathrm{CO})_{5}$, and $\mathrm{Mo}(\mathrm{CO})_{6}$, were explored in detail and found to produce unusual nanostructured inorganic materials via a reduction process [42]. In the past, Pd NPs have been made using a complicated sonication setup [43]-[45]; however, the NPs made in this report were made using a simple bath sonicator.

The next step is the solvothermal process, which allows these reactions to continue undisturbed by external factors until equilibrium is reached. Solvothermal process usually involves heating the solution (usually between $100^{\circ} \mathrm{C}$ and $1000^{\circ} \mathrm{C}$ ) [46] so that the stainless steel container creates an autogenous pressure that propels the reaction [47]. Here, as the reaction is initiated in the sonochemical process, the stainless-steel jacket creates a closed environment where the reactions can take place undisturbed. At this stage, once the metal nuclei were formed by sonication, these nuclei grow into NPs during the solvothermal process. The advantage of the design of the solvothermal process following the sonochemical process is that the reactions can take place at room temperature $\left(22-25^{\circ} \mathrm{C}\right)$, and do not need other external parameters to propagate the reaction. 
Several green chemistry principles were applied to this synthesis process, such as choosing environmentally benign chemical precursors, using safer solvents, applying energy-saving reaction conditions, and avoiding chemical derivatives. This process shows potential for synthesizing scalable catalysts suitable for large-scale treatment of VOCs in groundwater because of the ease in production of the catalyst as well as its potency. The catalysts synthesized by this technique have demonstrated the capability of very rapid and effective removal of TCE. To evaluate the real-world performance of these catalysts, they have been compared to a commercial Pd-on-GAC catalyst.
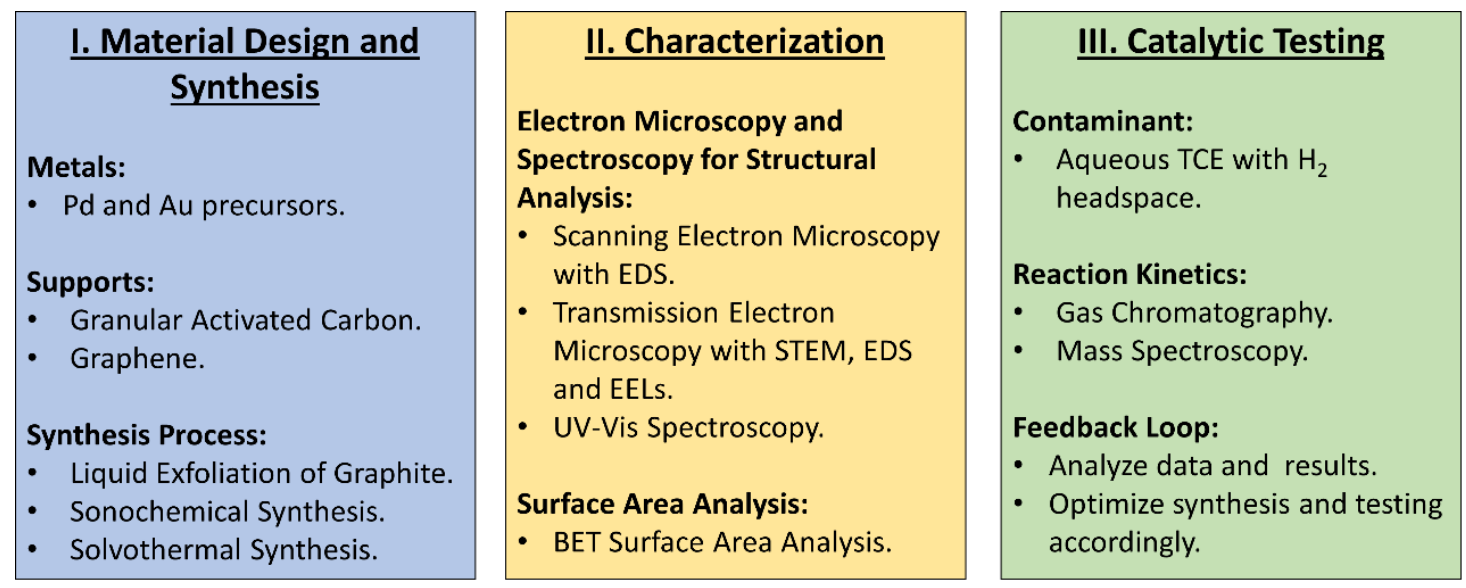

Figure 3. Detailed aspects of the carbon-supported Pd/Au NPs material synthesis, characterization and catalytic testing.

Building on the outline drawn in Figure 1, the details of the different aspects considered for this material system are listed in Figure 3. In this material system, the primary focus was on material design and synthesis, and characterization. Nevertheless, in order to optimize the catalysts' activity, some effort was spent in studying the reactivity and kinetics of TCE degradation. The material design, fabrication and characterization were carried out 
at Portland State University, while the catalytic reactivity testing was carried out with collaborators at Oregon Health \& Science University.

The economics of implementing this catalyst in real-life is not in the scope of this thesis, nevertheless, efforts are being made towards optimization of the material, specifically to address concerns of cost when using a heavy-metal catalyst. By using optimized quantities of $\mathrm{Pd}, \mathrm{Au}$ and GAC, a significant increase in lifetime can be achieved, as detailed in Section 2.4.5. In contrast, spent plain GAC that is commonly used to remove TCE in the industry, needs to be treated and replaced very often. This incurs several operational costs, and the risk of recontamination. Moreover, M. Reinhard et al. [48] have an extensive report stating that when TCE is present at or above $1 \mathrm{mg} / \mathrm{L}, \mathrm{Pd}$ catalysis becomes much more competitive than any other techniques for removal. They report that below $1 \mathrm{mg} / \mathrm{L}$, air stripping is the best way to remove TCE; however, as mentioned before, in this technique TCE is physically removed, but not broken down, requiring additional disposal. Next after air stripping, once again Pd-based catalysis was found to be the most cost-effective technique, for concentrations below $1 \mathrm{mg} / \mathrm{L}$. These reasons corroborate the need for a green, sustainable and effective carbon-supported $\mathrm{Pd} / \mathrm{Au}$ removal of TCE.

\subsection{Materials and Methods Used}

\subsubsection{Carbon Supports}

Granular activated charcoal (DARCO®, 20 - 40 mesh particle size, Sigma Aldrich, Catalog\# 242268) was used as a GAC support. When necessary, this GAC was crushed in a mortar and pestle and passed through a $70 \mu \mathrm{m}$ sieve to produce 200 mesh GAC particles. 
This smaller particle size of the support was chosen so the results could be comparable with previously reported materials [49].

Graphene was synthesized using liquid exfoliation, based on a modification of the process proposed by Y. Hernandez et al. [50]. In short, about $25 \mathrm{mg}$ of expandable graphite (ACS Materials, Product\# EG-170-400) was mixed with $50 \mathrm{~mL}$ n-methyl-2-pyrrolidone (NMP) (anhydrous, 99.5\%, Sigma Aldrich, Catalog\# 328634). This solution was probe sonicated (VCX-130, 1/4" 6mm probe) for 1 hour at $70 \%$ amplitude with 10 seconds on and 5 seconds off. This on/off setting was chosen to prevent heat buildup in the solution. After sonication, the solution was allowed to settle for $30 \mathrm{~min}$, so the heavier flakes could sink to the bottom. The top $20 \mathrm{~mL}$ of this solution then concentrated and washed in ethanol in a centrifuge (Legend Micro 21 Thermo Fisher Scientific Centrifuge) at 14,800 rpm for 15 mins and allowed to air dry overnight.

The carbon black (Fuel Cell Store, item \#590106) and graphite powder (Aldrich, Catalog\# 28,286-3) were used as-purchased.

The graphite nanoplates were synthesized by adjusting the process to make graphene, as described above. Expandable graphite (ACS Material, Product\# EG-170-400) was exfoliated in NMP with a probe sonicator in a ratio of 2:1 (volume of NMP, mL to weight of expandable graphite, $\mathrm{mg}$ ), for 1 hour at $85 \%$ amplitude with 10 seconds on and 5 seconds off. After allowing the solution to settle for $15 \mathrm{mins}$, the top half was centrifuged to retrieve the graphite nanoplates from the solution.

The procedure to make graphene differs from the graphite nanoplates procure in the following ways. For the liquid exfoliated graphene, the sonicated solution is allowed to rest 
for a longer period of time and a smaller amount of the supernatant solution is used. This process produces graphene that are few layered, electron transparent and of relatively good quality; while the graphite nanoplates produced are much thicker, with low quality including some defects. Hence, for applications, the liquid exfoliated graphene has better properties, but graphite nanoplates are more cost-effective.

\subsubsection{Synthesis of Carbon-Supported Pd/Au Nanoparticles}

A combination of sonochemical and solvothermal processes were used to make these NPs. The organometallic precursors used were palladium (II) acetate, $\operatorname{Pd}\left(\mathrm{O}_{2} \mathrm{CCH}_{3}\right)_{2}$, (reagent grade, 98\%, Sigma-Aldrich, Catalog\# 683124) and tetrachloroauric (III) acid trihydrate, $\mathrm{HAuCl}_{4} .3 \mathrm{H}_{2} \mathrm{O}$ (Acros Organics, Catalog\# AC411070050). A 1:1 (molar ratio) of $\mathrm{Pd}$ and $\mathrm{Au}$ was made by first sonicating $9 \mathrm{mg}$ of $\mathrm{Au}$ precursor in acetone $(99.9 \%$ Acetone, ACS Reagent Grade) for $15 \mathrm{~min}$. This was added to a $3 \mathrm{mg}$ of Pd (5 wt $\%$ loading) precursor in $20 \mathrm{~mL}$ acetone, which was then sonicated for $60 \mathrm{~min}$. A Bransonic 2510R-MT bath-sonicator was used for sonication. Then, $27 \mathrm{mg}$ of carbon substrate was added, and this solution was transferred to a Teflon liner in a stainless-steel autoclave. The autoclave was allowed to react at room temperature for 24 hours. No agitation, stirring or mixing was introduced to preserve the structural integrity of the support. After 24 hours, the catalyst samples were filtered and washed by centrifugation at 10,000 rpm for 5 min with fresh solvent and allowed to dry. Changes were made to the weights of the precursors, type of supports, synthesis time and temperature etc., specific to an experiment.

The commercial catalyst used for comparison was $5 \mathrm{wt} \% \mathrm{Pd}$ on activated wood carbon, (STREM Chemicals Inc., Catalog\# 46-1902). 


\subsubsection{Material Characterization}

A FEI Tecnai F20 TEM equipped with STEM and EDS capabilities that operates at an accelerating voltage of $200 \mathrm{kV}$, a FEI Sirion XL30 SEM with EDS and a Zeiss Sigma VP FEG SEM were used for the analysis of the catalyst. EELS data acquisition was carried out using a JEOL F200 STEM microscope equipped with a Gatan GIF Quantum IS that features a direct detection K2 camera capable of acquiring EELS data in counting mode, as well as low dose energy filtered and non TEM images at a frame rate up to 1600 images in in-situ mode [51]. A Shimadzu UV 3600 UV-Vis Spectrophotometer was used for sample adsorption measurements. The Quantachrome NOVA 2200e instrument was used to measure the samples' sorption and Brunauer-Emmett-Teller (BET) method was used to calculate their specific surface areas (SSA).

\subsubsection{Catalytic Reactivity Testing}

Each test was conducted with $20 \mathrm{mg}$ of catalyst, against $50 \mathrm{mg} / \mathrm{L}$ or $50 \mathrm{ppm}(1 \mathrm{mg} / \mathrm{L}=$ $50 \mathrm{ppm}$ ) of TCE in deionized water (DI water) in a batch reactor with crimp-sealed rubber septum stoppers. This concentration of TCE was chosen so the results could be compared with previously established rates [52], [53]. The catalyst was first added to an empty $150 \mathrm{~mL}$ glass vial followed by $120 \mathrm{~mL}$ of DI water. The vial was spiked for $20 \mathrm{~min}$ with 99.9\% hydrogen gas and then crimp sealed. Finally, $4.7 \mathrm{~mL}$ of a saturated TCE solution $(1.28 \mathrm{mg} / \mathrm{L})$ was injected at room temperature, and the vials were rotated at $50 \mathrm{rpm}$. The experiment was run either for 60 min or until the TCE was undetectable. In some cases,

once TCE was degraded below detection limits, another dose of TCE was injected (or "respiked") into the system and the headspace was again sampled at periodic intervals. For 
tests involving lower concentrations of TCE, $0.1 \mathrm{ppm}$ of TCE was injected into a $150 \mathrm{~mL}$ glass vial that had $120 \mathrm{~mL}$ of DI Water, dispersed catalyst and hydrogen headspace.

The reaction was monitored through headspace gas chromatography (GC) using an HP 5890 Series II GC equipped with a flame ionization detector (FID) and a DB-624 Agilent $\mathrm{J} \& \mathrm{~W}$ Scientific column (30 m long with $0.53 \mathrm{~mm}$ I.D.). When lower concentrations of TCE was used, an Electron Capture Detector (ECD) was used in the same GC system. Headspace TCE composition was converted back to the liquid-phase composition using standard calibration curves, assuming TCE partition concentration obeys Henry's law. Standards of known concentration TCE solutions were used to calibrate the peak area during individual GC runs. A sample with molar Pd:Au of 2:1 supported by $70 \mu \mathrm{m}$ GAC was tested at both 2 min and 20 min for identification of products using a GC equipped with a mass spectrometer (GC-MS) (Agilent 5975C MSD with a 7890A GC).

\subsection{Results and Discussion}

All the results presented below are of specimens made using a combination of the sonochemical and solvothermal process, described in the materials and methods section. These specimens were made with a molar ratio of 2:1 Pd:Au with acetone as solvent for a total of 24 hours. Any variations in the synthesis process have been specified in the particular section. In addition, while several synthesis parameters, such as molar Pd:Au ratios, type and size of supports, synthesis temperature, and reaction time were modified over 500 samples, only the most relevant results are presented here. 


\subsubsection{Liquid Exfoliated Graphene}

Graphene used in this study was made by modifying a previously established technique [50], and so has been briefly characterized here. Seen in Figure 4 (a), a low magnification TEM image shows the graphene produced is of several microns across in size. The Raman spectroscopy seen in Figure 4 (b) was taken on the sample shown in inset (c). The $D$ peak at $\sim 1350 \mathrm{~cm}^{-1}$ which relates to the defects in the sample and characteristic peaks of $G$ at $1580 \mathrm{~cm}^{-1}$ and $2 D$ at $\sim 2700 \mathrm{~cm}^{-1}$ are observable in this spectrum, indicating low defects but also indicating that the graphene produced is not single-layer [54]. However, this spectrum closely resembles analysis conducted on graphene made using liquid exfoliation by others in the past [55]. Therefore, the graphene synthesized using the technique presented in this study is low-defect, electron transparent and on-par with those made by other, similar liquid exfoliation methods.
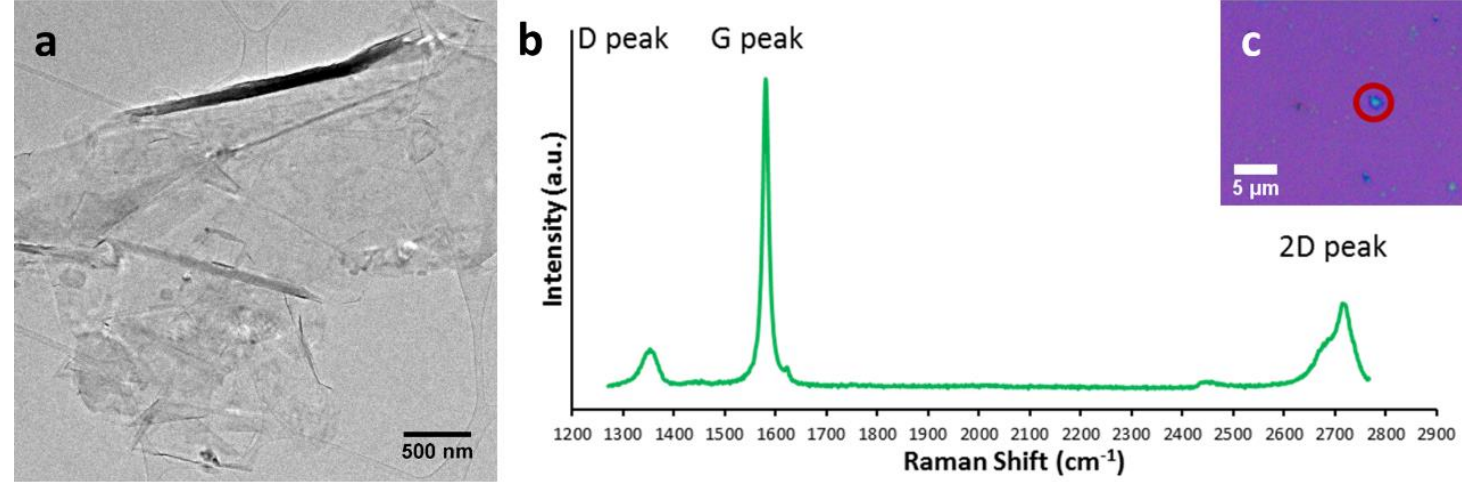

Figure 4. (a) Low magnification TEM of a graphene produced after liquid exfoliation, (b) Raman spectrum collected from sample, marked by the red circle as seen in inset (c). 


\subsubsection{Electron Microscopy characterization of Graphene and GAC supported Pd/Au NPs}

The structure and configuration of the as-made catalyst material was characterized using electron microscopy and spectroscopy techniques. First, the Pd/Au NPs with graphene as support was characterized, seen in Figure 5. Figure 5 (a) and (b) are SEM images of the same area on the specimen, imaged using both the secondary electron (SE) detector and the backscattered electron (BSE) detector. An area with high NP density was chosen for this analysis. In Figure 5 (a), the SE image the surface of the NP clusters can be seen, suggesting a rough morphology due to agglomeration of smaller NPs. As this detector collects signals produced due to the interaction of the electron beam and the surface of the specimens, the outer topographical structure of the NPs has been captured. In Figure 5 (b), the backscattered image is formed by the BSEs from deeper in the structure, due to the higher interaction volume, and reveals more Z-contrast but less surface information. This is because denser materials tend to scatter more than less dense materials, causing denser materials to appear brighter in BSE images in this image, the brighter core is more apparent, suggesting a heavier material is surrounded by a less-dense material. These two images show that the Au core is inside and surrounded by a Pd shell, therefore suggesting the $\mathrm{Pd}_{\text {Shell }} \mathrm{Au}_{\text {Core }}$ structure. This structure is further explained in Figure 5 (c), which is a schematic representation of this $\mathrm{Pd}_{\text {Shell }} \mathrm{Au}_{\text {Core }}$ structure, showing smaller Pd NPs agglomerate together to form an aggregate shell around an $\mathrm{Au}$ core. 

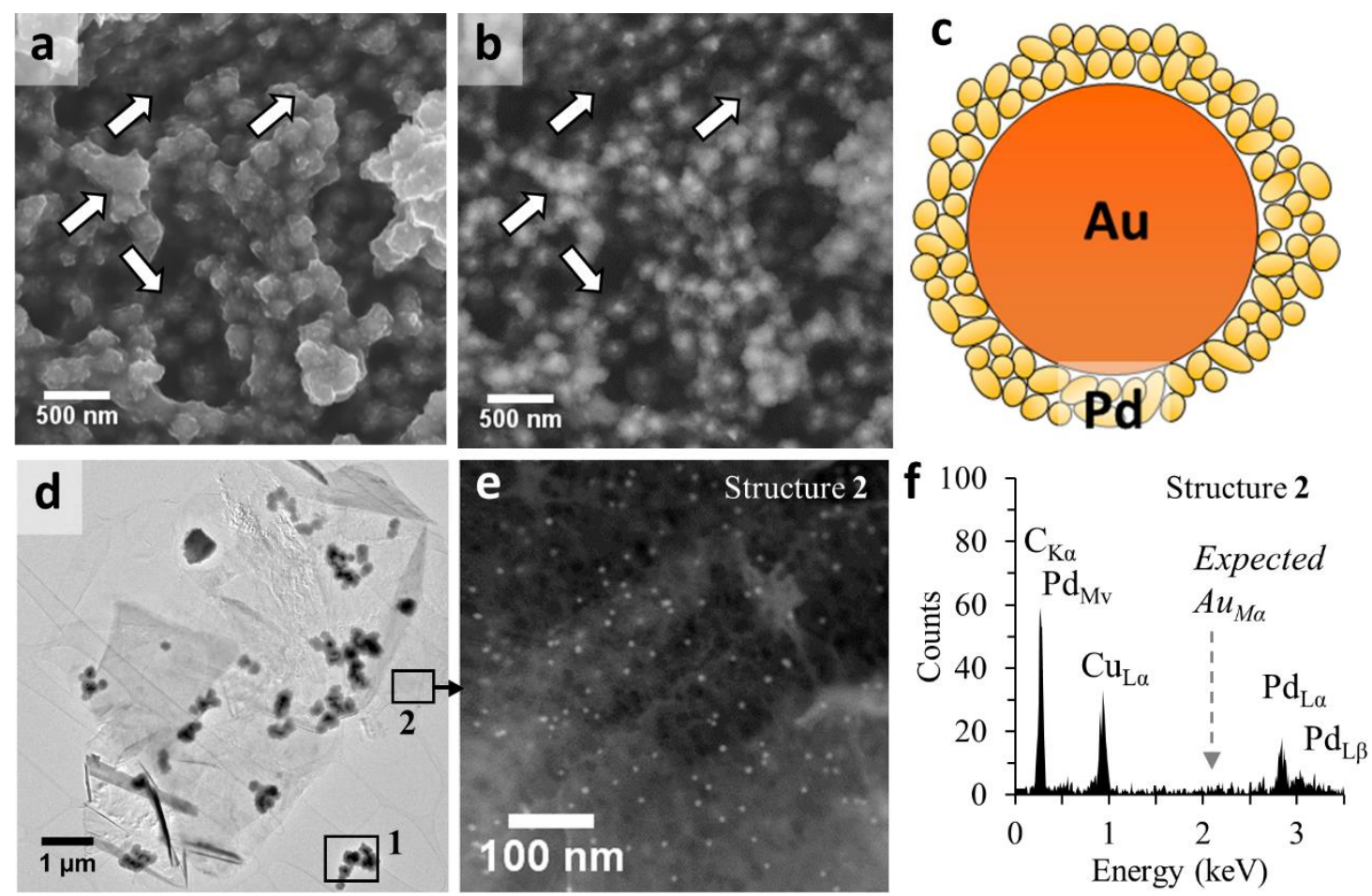

Figure 5. (a) SEM images with SE detector showing surface of NPs and (b) BSE detector showing contrast of metals making up the NPs. Arrows indicate regions of interest. (c) Schematic representation of PdshellAucore structure. (d) TEM image of Pd/Au NPs on graphene with two types of structures, Structure 1 and Structure 2, marked by boxes. (e) Dark-field STEM image of Structure 2 revealing small NPs dispersed evenly through graphene, EDS spectrum of this in (f) indicates strong Pd peaks but no Au, indicating the NPs are Pd on carbon support; $\mathrm{Cu}$ is from the grid. Structure 1 is examined in Figure 6.

Next, TEM characterization was carried out. Seen in Figure 5 (d), a low magnification image shows some clusters of NPs on a graphene sheet, along with some unsupported clusters around the graphene. These clusters, classified here as Structure 1, make-up a majority of the morphology of the sample. While the supported clusters are responsible for catalyzing the removal of TCE, the unsupported NP clusters do not play a role, as they are removed completely upon washing and cleaning of the catalyst after synthesis but before testing of the sample. However, here, these structures are useful for impartial 
characterization. Along with Structure 1, a secondary structure, classified here as Structure 2, was observed on the graphene support. Structure 2 is further analyzed in Figure 5 (e), a STEM dark field image of the graphene, where the bright spots are tiny NPs of $1-2 \mathrm{~nm}$ dispersed over the support.
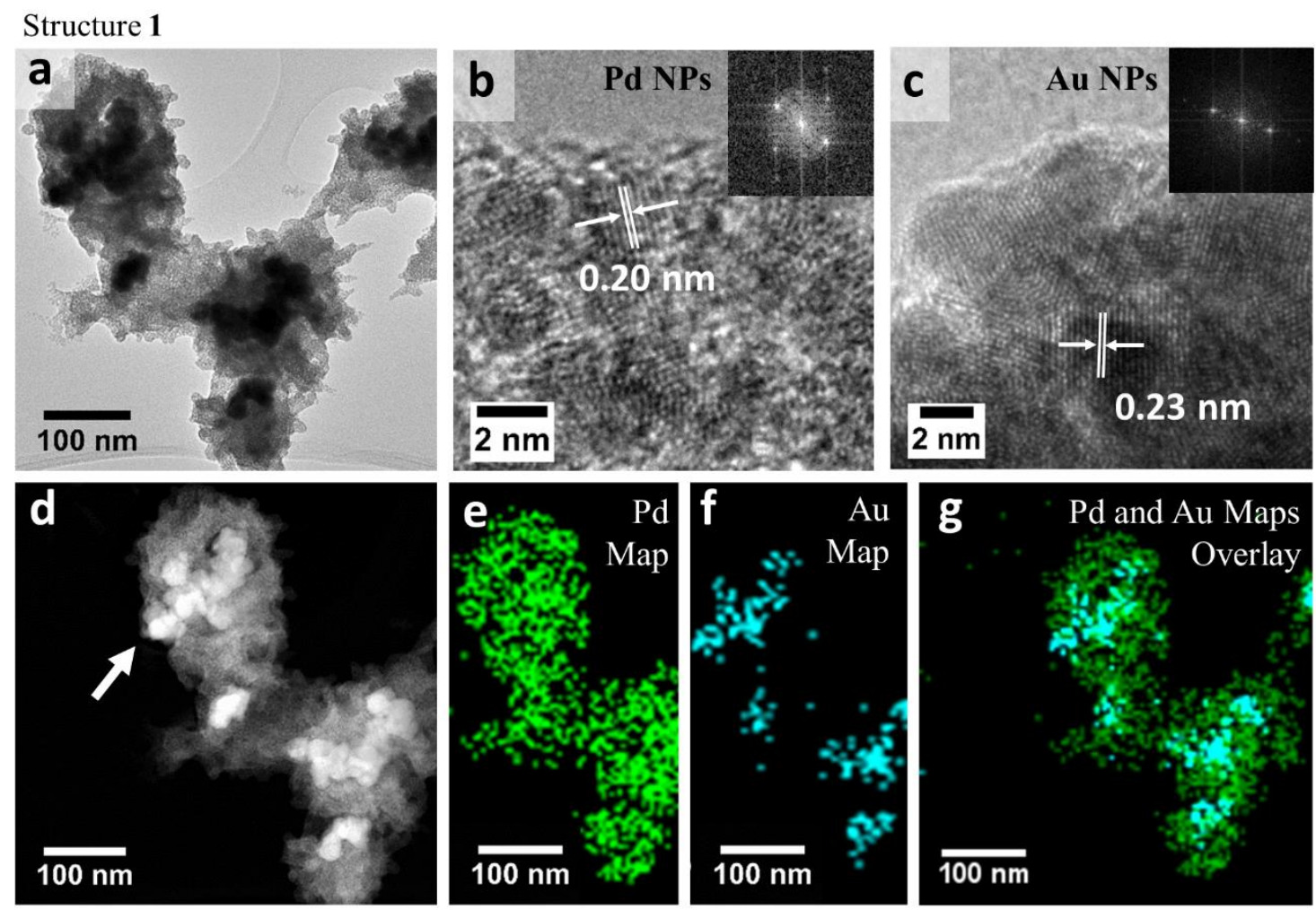

Figure 6. TEM analysis of Structure 1. (a) TEM image of a cluster with a less dense material surrounding a denser core. HRTEM images of (b) Pd and (c) Au NPs having observable lattice structures and the respective FFT insets show the NPs are crystalline. (d) STEM dark-field image of this cluster confirms two different materials with coreaggregated shell configuration. The arrow points towards an area where the aggregate Pd shell does not uniformly cover the Au core. EDS maps indicate core is Au (e), and aggregate shell is Pd (f) with (g) overlay confirming PdshellAucore structure.

An EDS spectrum of this area was taken, as shown in Figure 5 (f), indicating these NPs are made of Pd. The source of the $\mathrm{Cu}$ signal in this spectrum is from the TEM lacey carbon copper grid. Additionally, the peak identifying $\mathrm{Au}$ at $2.12 \mathrm{keV}$ is missing, as indicated in 
the spectrum. That is, Structure 2 is only made of Pd and has no Au in it. These tiny $\mathrm{Pd}$ NPs were found uniformly dispersed throughout the graphene support. This phenomenon indicates that $\mathrm{Pd}$ has some affinity to the carbon support.

Examples of Structure 1, the bimetallic NP cluster, were studied in detail in Figure 6. Both supported and unsupported clusters were studied, and no difference was observed between these; hence, results from both supported and unsupported clusters are presented here. Moreover, it is believed that the presence of Structure 2, consisting of small Pd NPs spread through the surface of graphene, prevents some of the clusters from hybridizing with the support. Figure 6 (a) is an image of an unsupported cluster indicating a core-shell configuration. The image contrast suggests that the NPs of darker contrast act as the core while the NPs of lighter contrast seem to form the shell surrounding the darker NPs. In fact, this structural configuration does not appear to be a traditional smooth-core-shell structure. Rather, both the denser and less-dense materials appear to be made up of smaller particles that have aggregated. This difference in density of the materials suggests a $\operatorname{Pd}_{\text {Shell }} \mathrm{Au}_{\text {Core }}$ configuration. A high resolution TEM (HRTEM) image of the smaller, individual Pd NP that form the shell, seen in Figure 6 (b), have lattice fringes, and the inset with the fast Fourier transform (FFT) suggest they are single crystalline in nature. The same is true for an exposed core, seen in Figure 6 (c), with the FFT inset also confirming crystallinity of these NPs. The crystallinity for the Pd and Au NPs is preferred over an amorphous phase, as crystallinity promotes better electron transport, which increases the overall activity of the catalyst [12]. 
The cluster in Figure 6 (a) was further analyzed in dark-field STEM mode, seen in Figure 6 (d). As can be seen, the image contrast in Figure 6 (a) is reversed comparing to that in Figure 6 (d). Both images confirm that the core is made of a denser material, which is different from the lighter material that forms the shell. The EDS elemental mapping, as shown in Figure 6 (e) and (f) of Pd and Au respectively. The overlay structure of these maps is seen in Figure $6(\mathrm{~g})$, revealing a $\mathrm{Pd}_{\text {Shell }} \mathrm{Au}_{\text {Core }}$ structure. The individual Pd NPs which agglomerate together to form the shell were found to have individual sizes in the range of $2-5 \mathrm{~nm}$ and the shell itself can measure up to $30 \mathrm{~nm}$. The Au core appear to be NPs in the sizes ranging from $30-50 \mathrm{~nm}$ that agglomerate to form up to $500 \mathrm{~nm}$ size centers. Hence, the overall sizes of the clusters can reach to micron scales. Detailed examination over several samples revealed that the Pd shell covering the Au is not very uniform, and on rare occasions, some portions of the Au cores remain exposed. One example of this can be seen in Figure 6 (d), with the arrow pointing to a location where the aggregate Pd shell does not uniformly cover the Au core.

The GAC samples were also characterized using the same techniques. Due to the high porosity and 3-dimensional structure of GAC, it is difficult to reveal detailed structural properties of the GAC-Pd/Au sample by TEM/EDS, especially for the $800-400 \mu \mathrm{m}$ GAC specimens. These materials were not characterized by the TEM due to their large sizes which prevents electron transmission through the samples. However, GAC particles smaller than $70 \mu \mathrm{m}$ were imaged by the TEM. Some of these results are shown in Figure 7. 

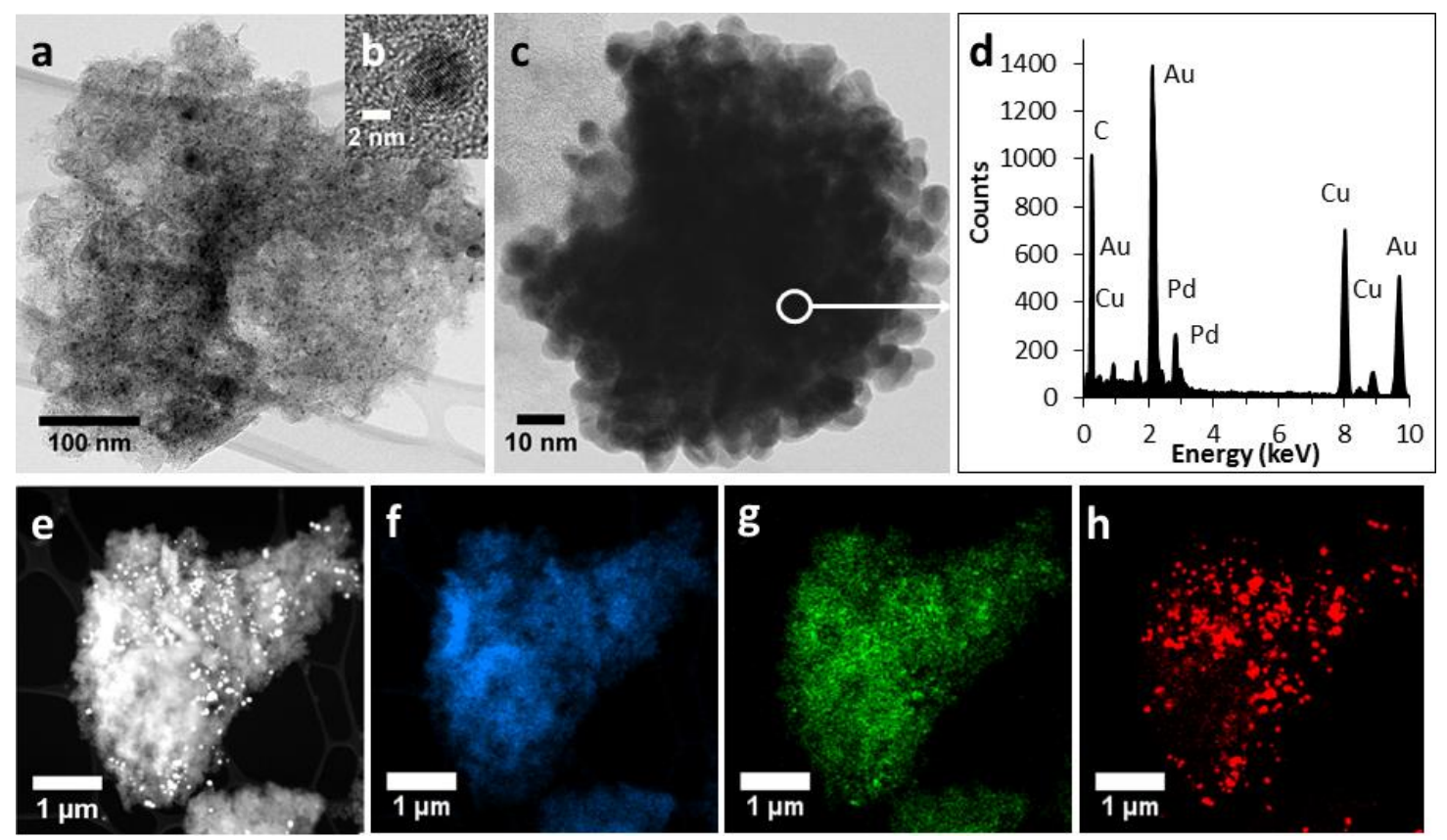

Figure 7. TEM analysis of (a) a GAC particle with inset (b) showing crystallinity of some smaller, embedded particles. (c) a larger cluster which appears be made of $\mathrm{Pd} / \mathrm{Au}$, according to the EDS spectrum in (d). (e) Dark-field STEM image of the GAC supported sample showing EDS maps of (f) carbon, (g) Pd and (h) Au.

Similar to the graphene-supported specimen in Figure 5 and Figure 6 , both large clusters and smaller, dispersed particles were observed in GAC-supported specimens. Note that Figure 7 (a) is a low magnification TEM image of one such GAC particle, showing dispersed NPs embedded throughout its structure. Inset Figure 7 (b) shows a smaller NP, the lattice structures of which demonstrate the crystalline nature. Numerous NPs like this were spotted throughout the GAC particle. A closer inspection of a clustered NP is seen in Figure 7 (c). The EDS spectrum in Figure 7 (d) revealed this particle to be a bimetallic $\mathrm{Pd} / \mathrm{Au}$ NP. The GAC particle seen in Figure 7 (e) was investigated under STEM/EDS, revealing maps of (f) carbon, (g) Pd, and (h) Au. Closer examination of these maps shows smaller Pd NPs were dispersed throughout the GAC particle as seen in Figure 7 (g), while 
the larger, accumulated Au NP formation is demonstrated in Figure 7 (h). There are common areas in both the Pd and Au maps in which the signals are overlapped. This indicates the presence of the larger clusters of Pd and $\mathrm{Au}$.

A notable feature of the GAC-supported Pd/Au NPs is that the NPs appear to be embedded throughout the GAC particle, not just restricted to the surface. This result suggests that Pd and Au deposit both on the surface and inside the pores of GAC. The formation of $\mathrm{Pd} / \mathrm{Au}$ NPs deep within the GAC pores is attributed to the porosity of GAC.

\subsubsection{Oxidation State of Pd NPs}

The oxidation state Pd is an important property for the catalysts, as it helps determine the decomposition pathway of TCE. This is because $\mathrm{Pd}^{0}$ is known to catalyze TCE through traditional HDH, in which dichloroethene isomers (1,1-DCE, trans-1,2-DCE, cis-1,2-DCE) and vinyl chloride (VC) are brief intermediate products [29]. On the other hand, if the Pd NPs has a different oxidative state, such as $\mathrm{Pd}^{+2}$ instead of $\mathrm{Pd}^{0}$, then different degradation routes can be expected, many of which include the formation of stable, unfavorable products that further pollute the environment [5].

Generally, EELS analyses have the advantages of spatially resolving spectral information into high-resolution maps and providing high energy resolution for the data collected. EELS analyses are particularly preferred for materials with low thickness, which is why this technique would be very useful to characterize the Pd/Au NPs. However, the traditional EELS acquisition introduces noise from multiple sources, including from electronic read-out of the $\mathrm{CCD}$ and the statistics of photon generation/collection. In the 
past, this high noise and low resolution of standard EELS detection prevented this technique from being used for such analyses.
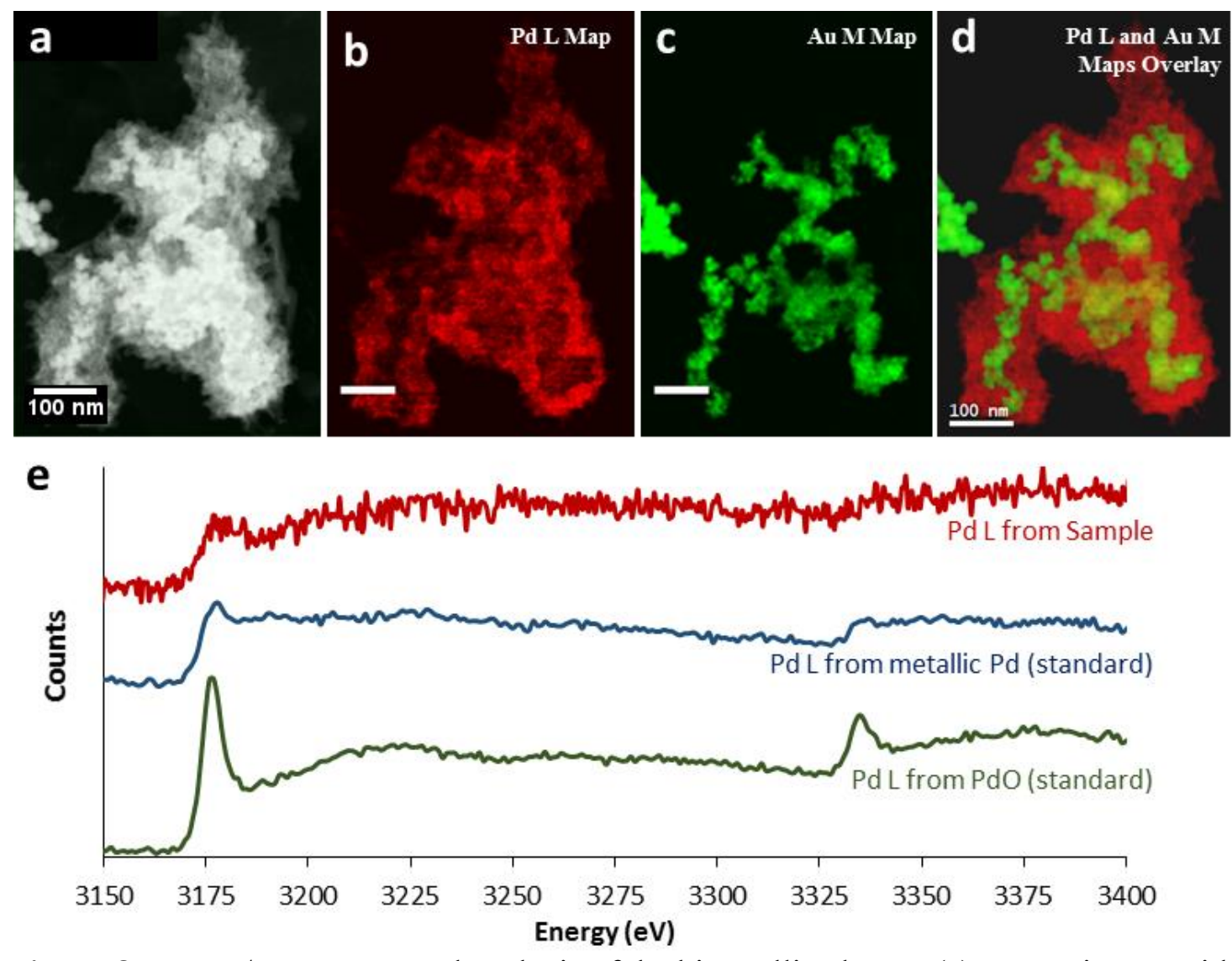

Figure 8. STEM/EELS map and analysis of the bimetallic cluster. (a) STEM image with EELS maps of (b) Pd L, (c) Au M and (d) overlaid maps showing a Pd ShellAuCore structure. (e) EELS spectrum from the sample compared with a metallic Pd standard and a $\mathrm{Pd}^{2+}$ standard. Upon comparison, the Pd spectrum from the sample was found to resemble the metallic standard.

The new design for direct detection of the $\mathrm{K} 2$ consists of a passivation/electronics layer, epilayer, and substrate leads to an increase in the signal-to-noise ratio and a reliability of analysis [51]. This new design of K2 allows for an improved EELS analysis can be carried out with detailed investigation on oxidation state of the NPs. To the authors' knowledge, 
this K2 direct detection EELS analysis has not been used to identify the oxidative state of such NPs in the past. Here, for the first time, this technique is used to demonstrate both the analysis of the material and effectiveness of the instrument used.

To identify if the NPs are in the elemental state or oxidized state, EELS was used on the same sample analyzed in Figure 5 and Figure 6. First, a STEM/EELS mapping was carried out using the conventional CCD camera. However, the data collected was too noisy and ambiguous. Next, the advanced K2 camera was used to collect both STEM images and the EELS spectrums, which are presented in Figure 8. Figure 8 (a) shows the STEM image that was mapped, revealing elemental Pd and Au maps, as seen in Figure 8 (b) and (c). These have been overlapped in Figure 8 (d) to confirm the $\operatorname{Pd}_{\text {Shell }} A u_{\text {Core }}$ structure. The EELS spectrum collected from this structure using the K2 camera is shown in Figure 8 (e). The top spectrum was taken from the sample, showing a Pd L edge. The lower two spectra are of Pd L from standards of a metallic Pd (chemical state 0$)$ and palladium oxide (PdO) (chemical state of $\mathrm{Pd}$ is $2+$ ). The spectrum from the sample most closely resembles the spectrum from the Pd metallic standard; therefore, the chemical state of Pd was thought to be 0 .

As no Au standards for the K2 camera EELS analysis are available at this time, this part of analysis is yet to be carried out. However, since the mechanisms for the formation of $\mathrm{Pd}$ and $\mathrm{Au}$ are very similar [42], $\mathrm{Au}$ is also expected to be zero-valent. The oxidative state of $\mathrm{Au}$, while an interesting characterization, has a secondary impact on the material's catalytic properties, since $\mathrm{Au}$ is a promoter of $\mathrm{Pd}$. The results discussed in Section 2.4.6 show that while Au itself does not degrade TCE, it promotes the catalytic ability of Pd to 
degrade TCE. Although it would be very interesting to use K2 EELS data to characterize the Au status in this sample, the detailed analysis performed by J. Bang, et al. [42] and Y. Mizukoshi et al. [43], [56] makes it clear that the oxidative state of Pd and Au would be the same as a result of the sonochemical process, and hence it is believed that Au has an oxidative state of $\mathrm{Au}^{0}$.

\subsubsection{Surface Area Characterization for GAC-based Materials}

When using GAC for catalysis, accessibility to the adsorption sites within the GAC pore structure and surface area availability is key [57]. Here, BET analysis was carried out to calculate SSA was carried out on all the GAC samples, including on a commercial Pd on GAC catalyst that was later used for comparison. Since pore-size and adsorption is significant feature of GAC, the analysis was carried out only on GAC and GAC-supported specimens.

Table 1: Specific surface area of catalysts calculated using BET analysis

\begin{tabular}{ll}
\hline Material & $\boldsymbol{a}_{\boldsymbol{s}}^{\prime}\left(\mathbf{m}^{\mathbf{2}} / \mathbf{g}\right.$ Cat $)$ \\
\hline $800-400 \mu \mathrm{m}$ GAC & 483.30 \\
$70 \mu \mathrm{m}$ GAC & 471.08 \\
$\mathrm{Pd} / \mathrm{Au}$ NPs on $800-400 \mu \mathrm{m} \mathrm{GAC}$ & 384.06 \\
$\mathrm{Pd} / \mathrm{Au}$ NPs on $70 \mu \mathrm{m} \mathrm{GAC}$ & 386.68 \\
$\mathrm{Pd}$ on $18 \mu \mathrm{m}$ GAC (Commercial) & 805.65 \\
\hline
\end{tabular}

The adsorption of TCE by GAC occurs primarily in the micropores, which are $7-10$ $\AA$ in size, where is it expected that majority of the TCE adsorption by GAC takes place. Upon characterization of samples, as seen in Figure 7, NPs were found reasonably 
dispersed all over the surface of this carbon support, including topographical features such as peaks and pores of the GAC. Hence, TCE removal can be expected to happen across the surface of the particle, which makes the SSA important.

There are some concerns with crushing a GAC particle to increase surface area. In general, by crushing a generic solid particle, the surface area of the smaller particles tends to increase due to the change in surface-to-volume ratio. In contrast, the BET analysis data provided in Table 1 shows the SSA of the $800-400 \mu \mathrm{m} \mathrm{GAC}$ and $70 \mu \mathrm{m}$ GAC samples are about the same. The SSA is reported in Table 1 as $a_{s}^{\prime}\left(\mathrm{m}^{2} / \mathrm{g}_{\text {Cat }}\right)$, surface area available per gram of the catalyst. The similarity of $a_{s}^{\prime}$ of the $800-400 \mu \mathrm{m} \mathrm{GAC}$ and $70 \mu \mathrm{m}$ GAC is attributed to the high porosity of the material, including the presence of embedded, deeply rooted pores throughout the structure. The majority of this SSA for larger particles is found in micropores (less than $2 \mathrm{~nm}$ in diameter). That is, the crushing does not influence the surface-to-volume ratio much due to the porosity of the particles. This could prove disadvantageous, as the NPs could block smaller pores and clog large pore pathways during synthesis. In addition, it is possible contaminants do not reach the insides of the pores (due to restricted mass transport), rendering the inner surface area ineffective. It is also possible that during the crushing of the larger particles, carbon dust causes pore blockage, leading to the further reduction of SSA available for adsorption and NPs support.

However, there are advantages to reducing the diameter of larger particles, which appear to out-weigh the disadvantages. Upon crushing the particle, the insides of the pore walls are brought to the surface, making them more accessible. This means the majority of the SSA of the $70 \mu \mathrm{m}$ GAC samples is available for NP deposition and for interaction with 
the contamination, which makes it a better support. This difference in SSA is further demonstrated in Section 2.4.6.

Another observable feature from Table 1 is the decrease in SSA between pure GAC and NP-supported GAC samples. This is because the BET technique relies on the sample adsorbing an inert gas, in this case nitrogen, to calculate SSA. While theoretically the surface area should increase with the addition of NPs, in this case the NPs block the surfaces and pores of GAC and stop the adsorption of nitrogen, hence decreasing the measurable SSA. This analysis is in good agreement with previously published results [58].

With the available information about the $70 \mu \mathrm{m}$ GAC and respective GAC-supported catalyst, the SSA of just the Pd/Au NPs on the surface of GAC was found to be about 84.4 $\mathrm{m}^{2} / \mathrm{g}$. This surface area is assumed to be the combination of the surface area of both the smaller NPs and the larger clusters.

\subsubsection{TCE Removal by Carbon-Supported Pd/Au NPs}

TCE Removal in Comparison to Commercial Pd-on-GAC

The kinetics of TCE removal in a hydrogen headspace and in the presence of the catalysts is presented in Figure 9. In this figure, the data is presented without fitting to provide a visual comparison, tracking the disappearance over time of the massconcentration $(C)$ normalized to initial mass-concentration of TCE $\left(C_{0}\right)$, represented as $C / C_{0}$. This TCE disappearance by as-made catalysts and the commercial $\mathrm{Pd}$ on GAC catalyst are compared in Figure 9 (a), with plain GAC as control. The loss in TCE by plain GAC is attributed to adsorption, as no products were detected. The disappearance is 
significantly faster with the $\mathrm{Pd} / \mathrm{Au} \mathrm{NPs}$ on graphene catalyst, due to the uniform distribution of NPs over the vast surface area of the support. In comparison, the commercial Pd on GAC catalyst and Pd/Au NPs on GAC appear to have similar rates of removal, despite the commercial catalyst having relatively smaller particle size $(18 \mu \mathrm{m})$.
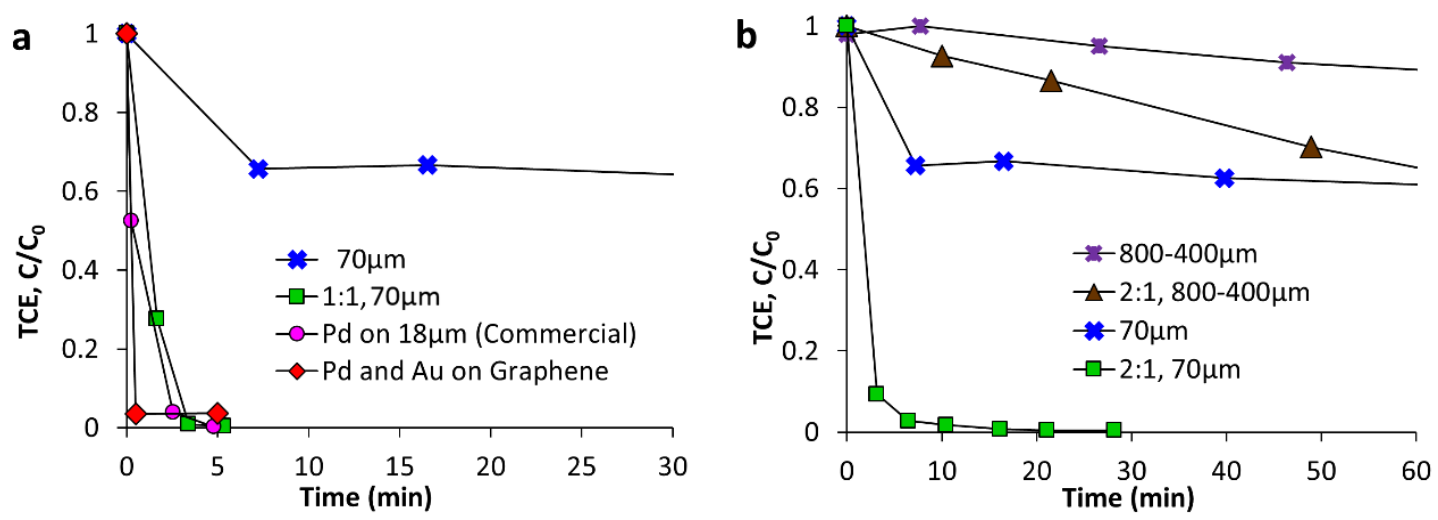

Figure 9. Concentration normalized $\left(\mathrm{C} / \mathrm{C}_{0}\right)$ TCE disappearance rates of (a) as-made catalysts, commercial Pd on GAC and plain GAC as control. (b) Significance of SSA of GAC-supported catalysts on TCE disappearance rate. All cases began with $50 \mathrm{ppm}$ of TCE in a hydrogen headspace.

\section{Effect of Surface Area of GAC on TCE Removal}

In Figure 9 (b), the disappearance of TCE by plain $70 \mu \mathrm{m} \mathrm{GAC,} 800-400 \mu \mathrm{m}$ GAC and respective catalysts. As described in Section 2.4.4, by crushing the GAC the SSA was increased, which is reflected in the change of adsorption of plain GACs. More significant is the difference between the GAC supported $\mathrm{Pd} / \mathrm{Au}$ NP catalysts. While the hybridization of the Pd/Au NPs $800-400 \mu \mathrm{m}$ sized GAC increases the TCE disappearance over the plain $800-400 \mu \mathrm{m}$ sized GAC, the rate of TCE disappearance of the Pd/Au NPs on the $70 \mu \mathrm{m}$ GAC is much more significant that its plain GAC counterpart. This is because for the smaller-sized catalysts, the increased SSA allows for a greater contact with the TCE. This 
causes a much faster disappearance rate, affirming that SSA plays a large role in the overall activity of these catalysts.

Longevity of Catalyst

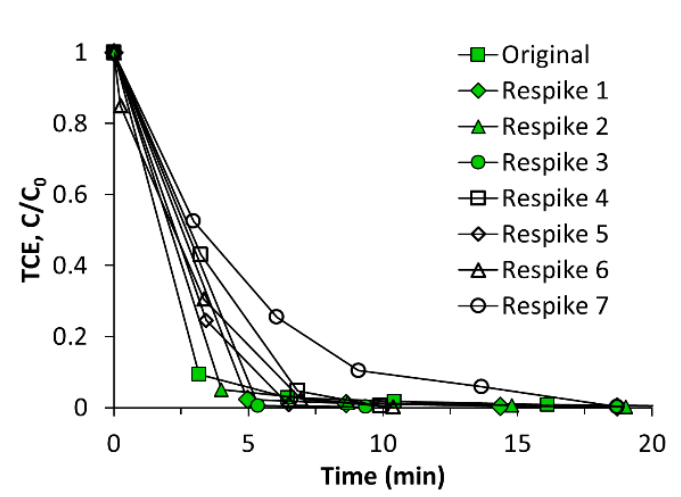

Figure 10. TCE disappearance when exposing the catalyst to consecutive doses of $50 \mathrm{ppm}$ of contaminant in a hydrogen headspace.
To test the longevity of the corresponding $\mathrm{Pd} / \mathrm{Au} \mathrm{NP}$ catalysts on GAC, respike experiments were performed and the results are seen in Figure 10. The disappearance of TCE was observed to be consistent over multiple respikes of $\mathrm{TCE}$, indicating the catalyst is both robust and stable against increasing concentrations of TCE.

At respike 7, the reaction begins to show signs of slowing down; however, the rate of disappearance is still rapid. It is important to note that at this point there is a change in headspace volume of the reactor due to the accumulation of the excess TCE solutions from previous runs, which has effected the rate of TCE disappearance.

\section{Role of Gold as Promoter}

In this section, the role of $\mathrm{Au}$ in this catalyst material is explored. It is known that $\mathrm{Au}$ by itself does not degrade TCE, since it is not a catalyst for HDH; however, Au acts as a promoter of hydrogen dissociation by $\mathrm{Pd}$. This is because $\mathrm{H}_{2}$ dissociation over $\mathrm{Pd}$ is more favorable with $\mathrm{Au}$ than with other materials, such as $\mathrm{Cu}$. $\mathrm{Au}$ is also considered to affect 
Pd's catalytic reactivity and selectivity due to its influence over Pd's electronic structure. Figure 11 (a) elucidates that Au NPs on GAC behave very similar to plain GAC in terms of interacting with TCE, confirming Au does not catalyze TCE disappearance. In both cases, the GAC appears to adsorb TCE without producing any detectable products. To further understand the effect of $\mathrm{Au}$ on $\mathrm{Pd}$ for this material, the molar ratio of $\mathrm{Pd}: \mathrm{Au}$ was varied between $1: 1,2: 1,150: 1$, and 300:1.
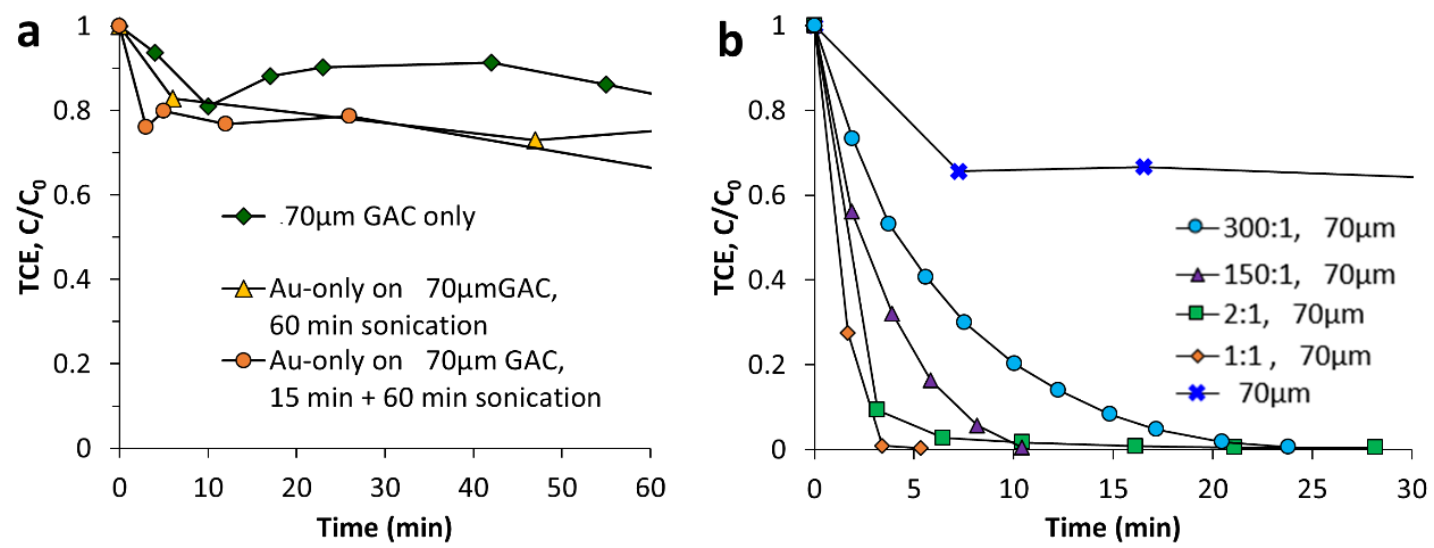

Figure 11. TCE disappearance with (a) Au only samples and GAC, (b) Disappearance by the catalyst at various molar Pd:Au loading on $70 \mu \mathrm{m} \mathrm{GAC}$, exposed to $50 \mathrm{ppm}$ of TCE in a hydrogen headspace.

For these materials, the amount of Pd was kept same while the Au was varied. The TCE removal rates are seen in Figure 11 (b). As expected, the more Au used with Pd, the faster the decomposition rate. This is in line with previous report, which suggest the contact between the $\mathrm{Pd}$ and $\mathrm{Au}$ atoms allows flow of electrons from $\mathrm{Pd}$ to $\mathrm{Au}$, making $\mathrm{Pd}$ more electron deficient and hence more active [12]. This means that the more the $\mathrm{Au}$, the more it influences Pd's catalytic activity. This outcome is highly beneficial, as it means that the 
catalyst can be optimized for specific applications where a compromise must be reached between catalyst performance and cost.

\section{Reduced Concentration of TCE}

All the tests performed thus far used a concentration of $50 \mathrm{ppm}$ of TCE against the catalyst. While this concentration shows the potency of the catalyst in removing such high concentration of contaminants, efforts were also made to study the catalyst behavior at lower concentrations of TCE. For this test, Pd/Au NPs supported by $70 \mu \mathrm{m}$

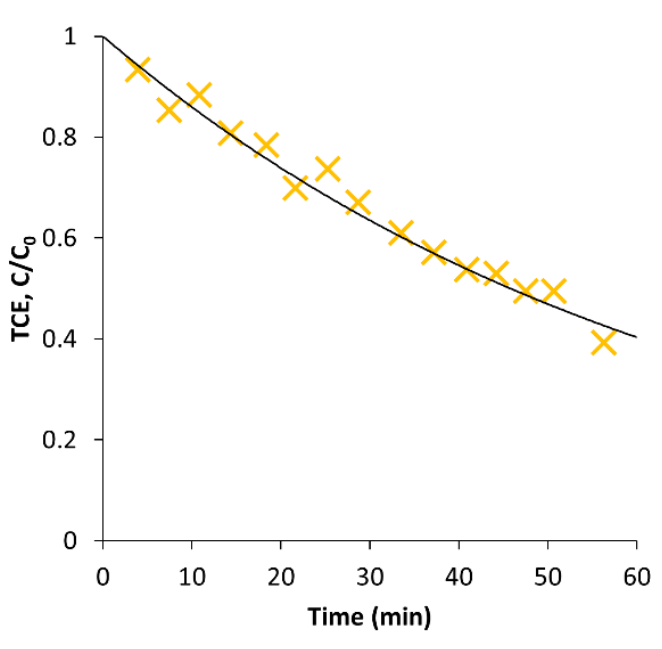

Figure 12. Pd/Au NPs on GAC degrading TCE at lower concentrations.

GAC was used with $0.1 \mathrm{ppm}$ TCE in a hydrogen headspace. The results from one such an experiment is shown in Figure 12 where the data has been plotted in yellow, with the black line showing an exponential fit for the data. While TCE disappearance is observed by this catalyst, the rate is much lower than expected. This is due to poor mass transfer in the reaction vessel which also lead to low repeatability. The rotation of the reactor was not enough for a uniform contact between TCE and the catalyst. A different design for the reactor system, like a flow reactor, would provide more accurate results of disappearance at this low concentration, as it promotes mixing. This work is in progress and is out of scope of the research presented here. 


\subsubsection{Kinetics and Mechanism for TCE Removal}

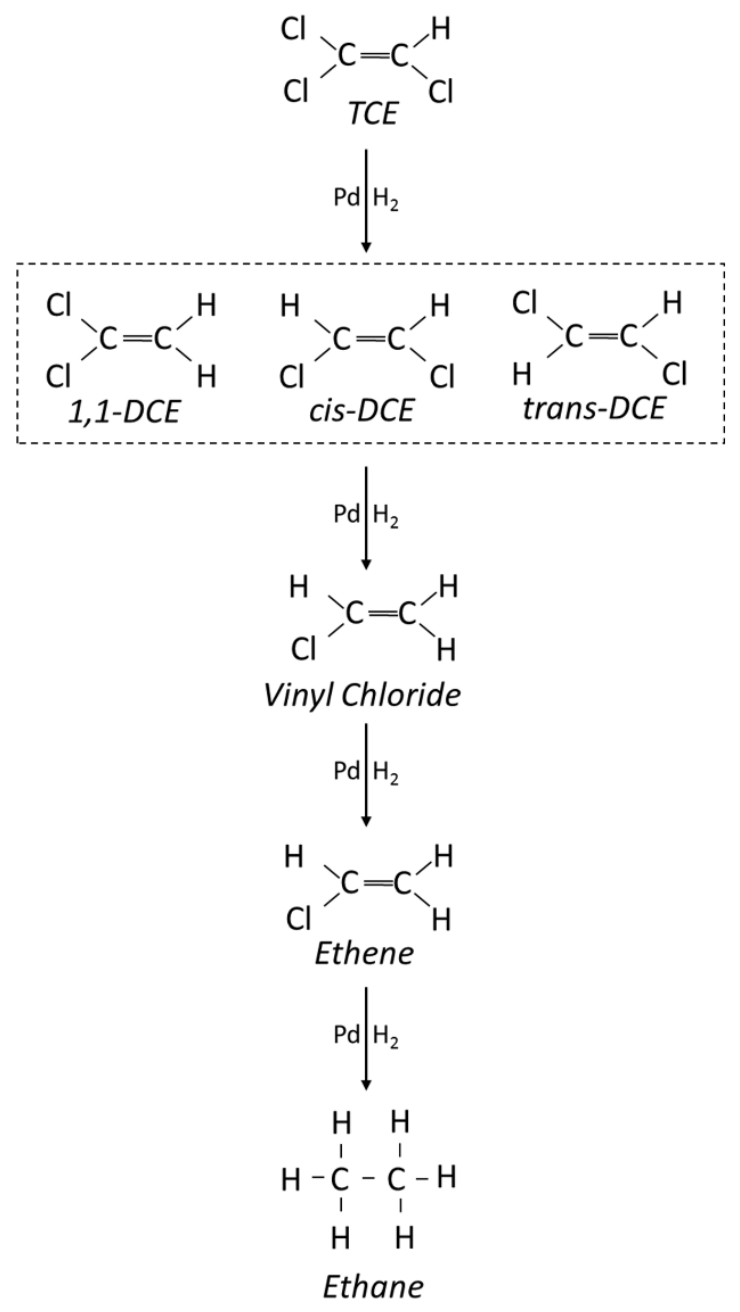

Figure 13. TCE removal via $\mathrm{HDH}$.
In this section, the kinetics of TCE decomposition has been studied. While a detailed analysis is out of scope for this thesis, an understanding of the mechanism of TCE breakdown has helped in improving the design and optimization of the catalyst. To identify the reaction pathway through which TCE was degraded in the presence of the carbon-supported $\mathrm{Pd} / \mathrm{Au}$ catalyst, a GC-MS was used to identify intermediate and product peaks.

2 min after the reaction started, intermediate products like 1,1-DCE, cis-DCE and trans-DCE, and VC were

detected. At $20 \mathrm{~min}$, no TCE or intermediate products were detected, affirming the formation of benign products like ethene and ethane.

This is consistent with the degradation of TCE via the traditional HDH, as reported by others [29], [59]. This path is elucidated in Figure 13. The discussion in Section 2.4.3 also supports this finding, as traditional HDH is the expected pathway for TCE disappearance in the presence of $\mathrm{Pd}^{0}$. 
Since traditional HDH has pseudo first-order kinetics [53], the TCE disappearance data collected in the previous section were assumed to be of this order fit to the equation (1) below,

$$
\frac{-d C_{T C E}}{d t}=k_{o b s} C_{T C E}
$$

where $C_{T C E}$ is the TCE mass concentration in the bulk fluid $\left(\mathrm{g}_{\mathrm{TCE}} / \mathrm{L}\right)$ and $k_{o b s}\left(\mathrm{~min}^{-1}\right)$ is the measured, observed (pseudo) first-order rate constant. The data fit was rigorously tested and validated, suggesting the rate of TCE removal by the catalysts developed here were also pseudo first-order.

To account for any differences in mass and SSA, the observed rates were normalized as

$$
k_{o b s}=k_{M} C_{P d}
$$

and

$$
k_{M}=k_{S A} a_{s}
$$

Where $k_{M}$ is the rate constant normalized for mass $\left(\mathrm{L} \mathrm{ged}^{-1} \mathrm{~min}^{-1}\right), C_{P d}$ is the mass concentration of Pd in each sample per reaction volume ( $\left(\mathrm{g}_{\mathrm{Pd}} / \mathrm{L}\right), a_{s}$ is the SSA of Pd NPs $\left(\mathrm{m}^{2} / \mathrm{g}_{\mathrm{Pd}}\right)$, and $k_{S A}$ is the rate constant normalized for surface area of $\mathrm{Pd}\left(\mathrm{L} \mathrm{m}^{-2} \mathrm{~min}^{-1}\right)$. In these experiments, the reaction volume was defined as the volume of the solution (120 $\mathrm{mL}$ ) as no reaction was expected to occur in the headspace.

The experiments were designed to keep $C_{P d}$ constant across all samples at a value of 5 wt $\%$ loading, or $0.0083 \mathrm{~g} / \mathrm{L}$. Here, $k_{S A}^{\prime}$ is reported, defined as $k_{M}$ normalized to SSA of 
catalyst $a_{s}^{\prime}\left(\mathrm{m}^{2} / \mathrm{g}_{\mathrm{Cat}}\right)$, if the surface area per gram of $\mathrm{Pd}\left(\mathrm{m}^{2} / \mathrm{g}_{\mathrm{Pd}}\right)$ is a constant for the respective samples. Hence, $k_{S A}^{\prime}$ has the units $\left(\mathrm{L} \mathrm{g}_{\mathrm{Cat}} \mathrm{g}_{\mathrm{Pd}}{ }^{-1} \mathrm{~m}^{-2} \mathrm{~min}^{-1}\right)$.

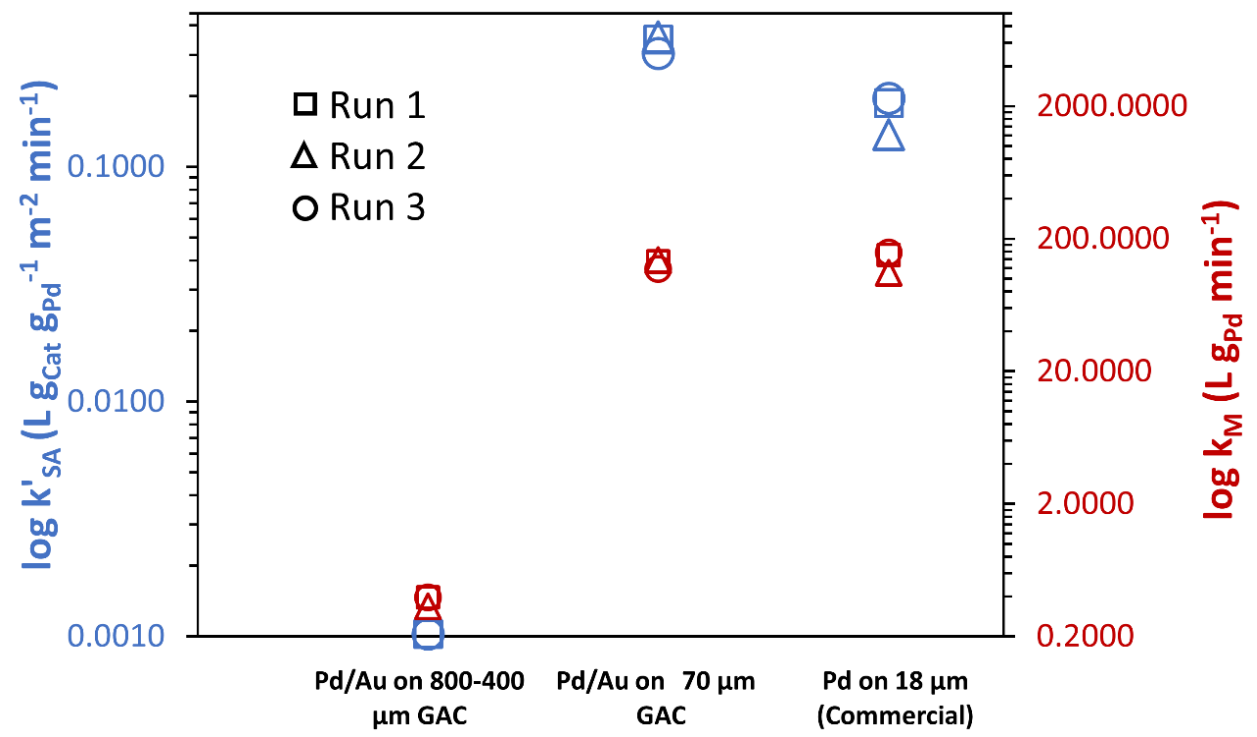

Figure 14. TCE removal rates for $\mathrm{Pd} / \mathrm{Au}$ catalysts on $800-400 \mu \mathrm{m} \mathrm{GAC}$ and on $70 \mu \mathrm{m}$ GAC as compared to a commercial catalyst. The $k_{M}$ is the degradation rate normalized to the mass concentration of $\mathrm{Pd}$, while the $k_{S A}^{\prime}$ is the rate normalized to the SSA of the catalyst (with units $\mathrm{L} \mathrm{g}_{\mathrm{Cat}} \mathrm{g}_{\mathrm{Pd}}^{-1} \mathrm{~m}^{-2} \mathrm{~min}^{-1}$ ).

The TCE removal data shown in Figure 9, with two additional repeat runs, were fit and analyzed as per equations (1), (2), and (3) mentioned above. The least-square minimization was used on this first-order degradation model. The rates calculated are plotted in Figure 14 and tabulated in Table 2 for convenience. Based on the data, it was found that for TCE decomposition, the $70 \mu \mathrm{m}$ GAC supported Pd/Au NPs catalyst, with a $k_{S A, A v g}^{\prime}$ of $0.34 \mathrm{~L} \mathrm{~g}_{\text {Cat }}$ $\mathrm{gPd}^{-1} \mathrm{~m}^{-2} \min ^{-1}$ is about 2 times as fast as the commercial Pd on GAC, which has a $k_{S A, A v g}$ of $0.17 \mathrm{~L} \mathrm{~g}_{\mathrm{Cat}} \mathrm{gPd}^{-1} \mathrm{~m}^{-2} \mathrm{~min}^{-1}$. 
Table 2: Observed and Normalized TCE Decomposition Rates

\begin{tabular}{|c|c|c|c|}
\hline & $\begin{array}{c}\text { Pd/Au on } 800-400 \\
\mu \mathrm{m} \mathrm{GAC}\end{array}$ & $\begin{array}{c}\text { Pd/Au on } 70 \mu \mathrm{m} \\
\text { GAC }\end{array}$ & $\begin{array}{c}\text { Pd on } 18 \mu \mathrm{m} \text { GAC } \\
(\text { Commercial })\end{array}$ \\
\hline \multirow{3}{*}{$\begin{array}{c}\boldsymbol{k}_{\boldsymbol{o b s}} \\
\left(\mathrm{min}^{-1}\right)\end{array}$} & 0.0033 & 1.1240 & 1.1255 \\
\hline & 0.0028 & 1.1520 & 0.9200 \\
\hline & 0.0033 & 0.9820 & 1.3140 \\
\hline $\begin{array}{l}k_{o b s, A v g} \\
\left(\mathrm{~min}^{-1}\right)\end{array}$ & $0.003 \pm 0.0$ & $1.09 \pm 0.09$ & $1.16 \pm 0.21$ \\
\hline \multirow{3}{*}{ 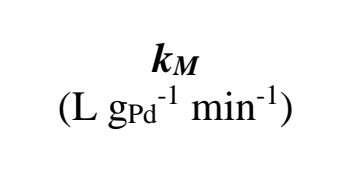 } & 0.3948 & 134.9124 & 150.5892 \\
\hline & 0.3372 & 138.2796 & 110.4048 \\
\hline & 0.3756 & 117.8556 & 157.7352 \\
\hline 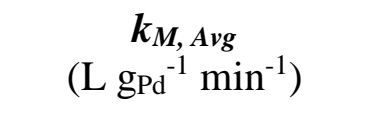 & $0.38 \pm 0.03$ & $130 \pm 11$ & $139 \pm 26$ \\
\hline \multirow{3}{*}{$\begin{array}{c}k_{S A}^{\prime} \\
\left(\operatorname{L~g~gat~}_{\mathrm{g}_{\mathrm{Pd}}^{-1}} \mathrm{~m}^{-2} \mathrm{~min}^{-}\right.\end{array}$} & 0.0010 & 0.3489 & 0.1869 \\
\hline & 0.0009 & 0.3576 & 0.1370 \\
\hline & 0.0010 & 0.3048 & 0.1732 \\
\hline 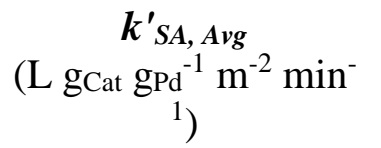 & $0.0010 \pm 0.0$ & $0.34 \pm 0.03$ & $0.17 \pm 0.03$ \\
\hline
\end{tabular}

Finally, given that the surface area of the Pd/Au NPs on the carbon-supported Pd/Au NPs specimen was calculated at $84.4 \mathrm{~m}^{2} \mathrm{~g}^{-1}$ in Section 2.4 .4 , the $k_{S A}$ for this material is $1.52 \pm$ $0.17 \mathrm{~L} \mathrm{~m}^{-2} \mathrm{~min}^{-1}$. However, since no such information is available for the commercial catalyst, a comparison cannot be made with the commercial catalysts on the basis of $k_{S A}$.

\subsubsection{Mechanism of Formation of Carbon-Supported Pd/Au NPs}

While much has been discussed about the catalyst such as its basic structure and activity against TCE, little has been discussed about the mechanism behind the catalyst formation and its behavior. In an attempt to further understand the cause behind the catalyst behavior, 
the following analyses were carried out. In this section, the focus is on the formation and growth of the Pd/Au NPs. For these experiments, graphene was used as the primary support for consistency and ease in TEM characterization. However, where appropriate, GAC was used. Moreover, a detailed study of the carbon supports with the Pd/Au NPs has been conducted in Section 2.4.8.

Here, the results are organized as first the study of interaction between $\mathrm{Pd}, \mathrm{Au}$ and carbon support, then the effects of variations in sonochemical process (by varying solvent and sonication times), and finally effects of variations in solvothermal process (by varying synthesis time and temperature). At the end of this section, a brief mechanism for the formation of the NP clusters is discussed.

\section{Interaction between $\mathrm{Pd}$, Au and Carbon Support}

To study the interactions between the metals and their support, specimens of individual metal NPs on graphene were made. The procedure to make the samples were the same; that is, the precursors were mixed with acetone and sonicated, except that only one metallic precursor (either palladium (II) acetate or tetrachloroauric (III) acid trihydrate) was used per sample. The TEM images of these samples are shown in Figure 15. Images Figure 15 (a) and (b) are of Pd NPs on graphene, and (c) and (d) are of Au NPs on graphene. Figure 15 (a) is a relatively low magnification image of Pd NPs on graphene, showing the Pd NPs uniformly dispersed over the graphene surface. The arrangement of Pd NPs on the graphene is very similar to Structure 2 (composed of tiny Pd NPs spread over the graphene) seen in Figure 5 (e); except the NPs in this sample are larger. Based on these observations, it is believed that in the absence of $\mathrm{Au}$, only Structure 2 is formed, and these NPs grow in 
size instead of forming clusters. Figure 15 (b) is a HRTEM image in which the lattice structures of the NPs are visible, indicating the NPs are single crystalline.

For the sample with Au only, similar observations were made. Figure 15 (c) is a relatively low magnification image of $\mathrm{Au}$ on graphene. Here, a sparse dispersion of the NPs is observed. In fact, for these images, the concentration of the Au precursor was increased by a factor of about thirteen, from $0.23 \mathrm{mg} / \mathrm{mL}$ to $3 \mathrm{mg} / \mathrm{mL}$. When the original concentration was used, although a color change was detected in the forming solution (indicating a reaction had occurred), no NPs were observed on the graphene.

In addition, despite a high concentration of precursor used, very few small, crystalline $\mathrm{Au}$ NPs in the range of $5-10 \mathrm{~nm}$ were formed on the graphene, as seen in Figure 15 (c). The HRTEM image in Figure 15 (d) shows these NPs are also crystalline. The lack of NPs despite a change in color of the solvent suggests Au has a weak interaction with graphene, as very few NPs accumulate on the graphene. This is in agreement with a previous report [12] that used DFT simulations, in which Au was shown to have a much weaker interaction with graphene than that of Pd.

In both samples, no large clusters were observed, indicating that clusters form only when both metals are present, with Pd on the outside and Au on the inside. This suggests that the formation of the $\mathrm{Pd} / \mathrm{Au}$ cluster in our experiments is due to attractive interactions between Pd and Au atoms, which causes them to spontaneously move towards each other, as described previously [12]. In addition, these experiments demonstrate that Pd has a great affinity for graphene, which explains why the Pd/Au NP clusters form over graphene. 


\section{Pd NPs on Graphene}
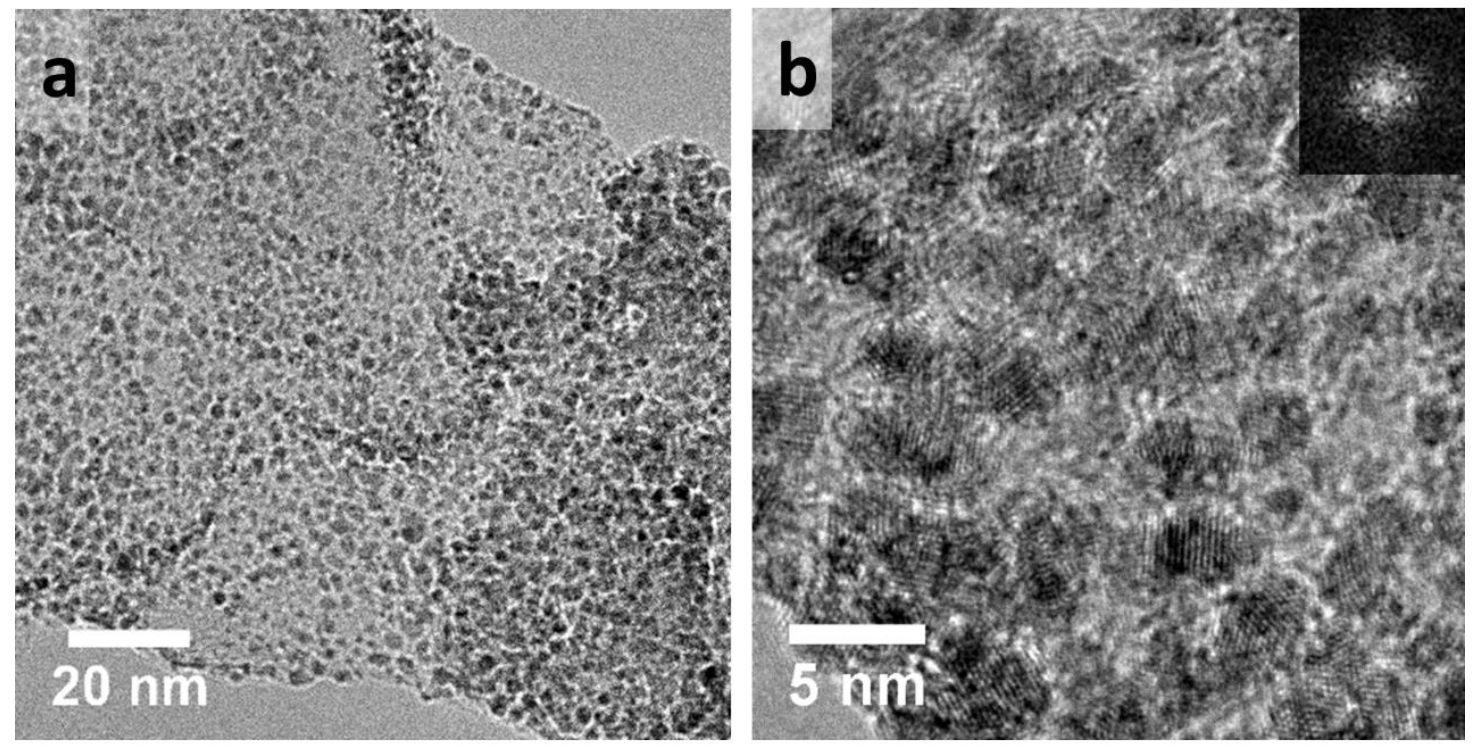

Au NPs on Graphene

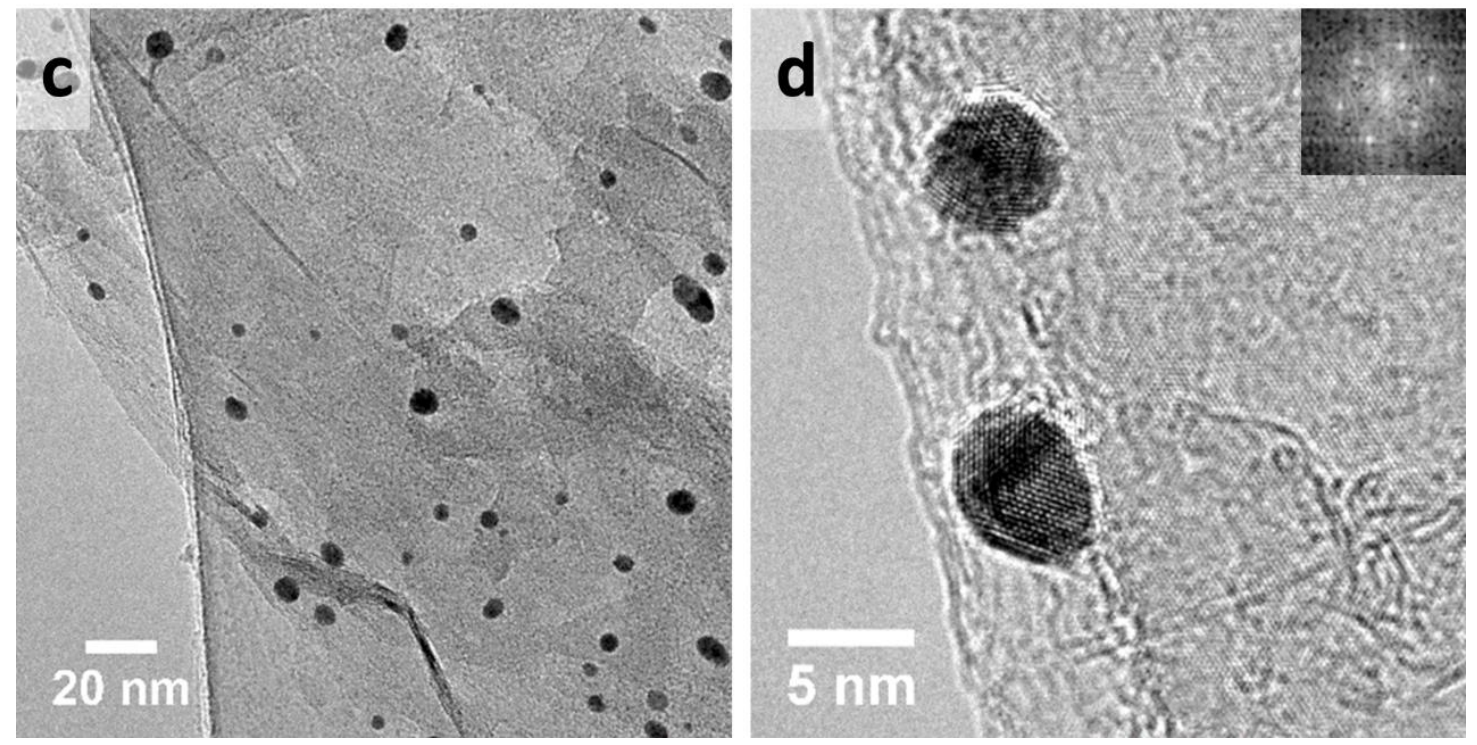

Figure 15. TEM images of (a) Pd NPs on graphene at a low magnification and (b) HRTEM image showing crystallinity of NPs. (c) Low magnification of Au NPs showing sparsely scattered NPs over support and (d) HRTEM image showing crystallinity of these NPs.

While Pd has a great affinity for both graphene and $\mathrm{Au}$, Au has little affinity for the support. Hence, when both metals and support are present, the Pd clusters around the $\mathrm{Au}$, 
forming Structure 1, and individually forms on the graphene support, forming Structure 2. This affinity to graphene also causes some of the clusters to form on the support; however, the abundance of Structure 2 might limit the formation of clusters on the surface of graphene, leading to formation of both supported and unsupported clusters.

Interesting, all clusters were found to have an aggregate $\mathrm{Pd}_{\text {Shell }} \mathrm{Au}_{\text {Core }}$ configuration, but not the reverse. This is because, in previous reports, the $\mathrm{Pd}_{\text {Shell }} \mathrm{Au}_{\text {Core }}$ configuration has been found to have a higher surface energy stability over the $\mathrm{Au}_{\text {Shell }} \mathrm{Pd}_{\text {Core }}$ structure [60]. Conforming to this, no $\mathrm{Au}_{\text {Shell }} \mathrm{Pd}_{\text {Core }}$ structures were observed in any of the materials synthesized in this project.

\section{Effect of Sonication on Formation of NPs}

The formation of noble metal NPs with chemical state 0 was explored in the past by [42]. It was found that during sonication, sonolysis of water occurs which generates $\mathrm{H}$. radicals. These radicals were found to act as reductants to form zero-valent Pd/Au NPs. The $\mathrm{AuCl}^{4-} / \mathrm{Au}^{0}$ and $\mathrm{Pd}^{2+} / \mathrm{Pd}^{0}$ reactions occur when precursors dissolve in the solvent and are reduced to form Au and Pd NPs. The difference in redox potential of the reactions, $\mathrm{AuCl}^{4-} / \mathrm{Au}^{0}(1.002 \mathrm{~V})$ and $\mathrm{Pd}^{2+} / \mathrm{Pd}^{0}(0.915 \mathrm{~V})$, is assumed to be partly responsible for the $\mathrm{Pd}_{\text {Shell }} \mathrm{Au}_{\text {Core }}$ configuration, causing the reduction of $\mathrm{Pd}$ and $\mathrm{Au}$ to occur sequentially rather than simultaneously. The most positive redox potential is of $\mathrm{AuCl}^{4-} / \mathrm{Au}^{0}$, hence $\mathrm{Au} \mathrm{NPs}$ form first followed by Pd NPs.

Based on previous reports [42], [56], [60], the following reactions are thought to occur

during the sonochemical process. In some cases, the solvent or organic additives were 
found to promote the formation of the metals, these are referred to as $\mathrm{RH}$ in the equations below:

Ionization in solvent:

$$
\begin{aligned}
& \mathrm{HAuCl}_{4} \rightarrow \mathrm{H}^{+}+\mathrm{AuCl}_{4}^{-} \\
& \mathrm{Pd}\left(\mathrm{O}_{2} \mathrm{CCH}_{3}\right)_{2} \rightarrow \mathrm{Pd}^{2+}+2\left(\mathrm{O}_{2} \mathrm{CCH}_{3}\right)^{-1}
\end{aligned}
$$

Sonolysis of water:

$$
\begin{aligned}
& \mathrm{H}_{2} \mathrm{O} \rightarrow \mathrm{H} \cdot+\mathrm{OH}^{\cdot} \\
& \mathrm{H} \cdot+\mathrm{H} \cdot \rightarrow \mathrm{H}_{2} \\
& \mathrm{H} \cdot+\mathrm{OH} \cdot \rightarrow \mathrm{H}_{2} \mathrm{O} \\
& \mathrm{RH}+\mathrm{OH} \cdot(\text { or } \mathrm{H} \cdot) \rightarrow \mathrm{R}+\mathrm{H}_{2} \mathrm{O}\left(\text { or } \mathrm{H}_{2}\right)
\end{aligned}
$$

Reduction of metal ions:

$$
\left.\mathrm{AuCl}^{4-} \text { or } \mathrm{Pd}^{2+}+\mathrm{H} \cdot(\text { or } \mathrm{R} \cdot) \rightarrow \mathrm{Au}^{0} \text { or } \mathrm{Pd}^{0}+\mathrm{H}_{2} \text { (or } \mathrm{R}\right)
$$

Equations (4), (5) are of the ionization of the precursors in the solvent. Equations (6), (7) and (8) deal with the sonolysis of water that generates the $\mathrm{H} \cdot$ and $\mathrm{OH} \cdot$ radicals. If the solvent or organic additive participates in the reaction, then equation (9) is expected to take place. Finally, in equation (10), the reduction of the metal ions occurs, leading to the formation of $\mathrm{Pd}^{0}$ and $\mathrm{Au}^{0}$.

In order to study the effects of sonication, the following experiment was carried out. Both the Pd and Au precursor solutions were sonicated for the same amount of time, 
together. In the original synthesis method, Au precursor solution is given an extra 15 min of sonication before adding the Pd precursor, giving Au a total of 75 min of sonication, while the Pd is only sonicated for $60 \mathrm{~min}$. If the redox potential was indeed driving the formation of the NPs, then Au would have a small head start. However, since the difference in redox potentials of the metals is so low, it is more likely that the samples would end up competing with each other in formation of NPs.
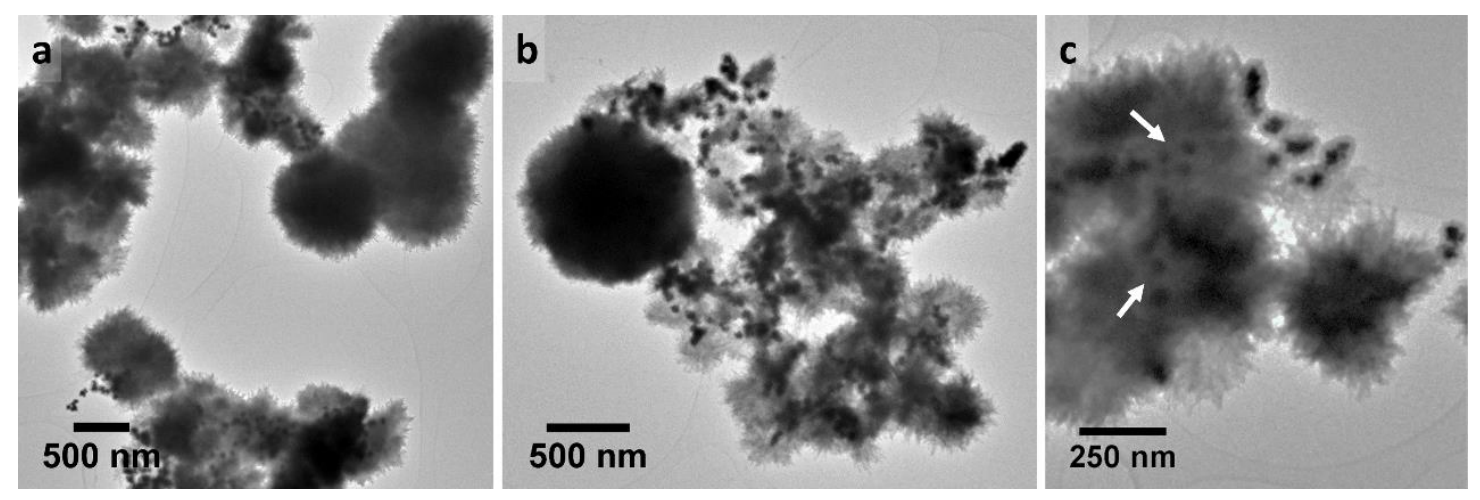

Figure 16. TEM of a sample in which both the Pd and Au precursors were sonicated and allowed to react for the same amount of time, producing non-uniform bimetallic clusters. Images (a), (b) and (c) were taken on different locations of the same sample. Arrows point to areas revealing the smaller Au cores deep inside the thicker Pd aggregate shells.

The results of this experiment are shown in Figure 16. For simplicity, these samples were made without the graphene support. Figure 16 (a), (b), and (c) were taken at different locations of the same sample, showing a non-uniform formation of the core-shell structures. As expected, both $\mathrm{Pd}$ and $\mathrm{Au}$ were forming almost at the same time, with $\mathrm{Au}$ having a small lead, leading to clusters of varied shapes and sizes. Certain areas appear to have a shallow layer of Pd NPs covering Au cores, while in other locations, Pd NPs form a thick, dense layer around the Au core. Thus, it can be inferred that in the original synthesis, the additional $15 \mathrm{~min}$ of sonication for the Au allows for the formation of a stable 
core that the Pd NPs can surround. That is, these results prove that while the difference in redox potentials of the reactions is not solely responsible for the core-structure, it definitely plays a role in this formation.

Effect of Solvent on the Formation of NPs

The solvent used for the synthesis can often play an important role in the formation of the NP clusters during the sonochemical process, as seen in equations (9) and (10). When choosing solvents, an important factor is the ionization of the metallic precursors in the solvent, seen in equation (4) and (5). That is, the precursor needs to first ionize in the solution, and this metal ion gets reduced in equation (10). Therefore, a solvent that promotes ionization of the precursors is highly preferable. While DI water seems to be a very promising solvent for this process, during the experiments, the precursors were found to be unevenly dissolved in the water. Despite magnetic mixing and other types of agitation, the amount of precursor seemed to vary every time, which effected all subsequent cluster formation steps. Thus, reproducibility was poor for these samples and the results are not included here.

Thus far, acetone was used as the solvent to make carbon-supported $\mathrm{Pd} / \mathrm{Au} \mathrm{NPs}$ materials. These samples were compared against samples made using ethanol as a solvent.

Ethanol has several advantages as a solvent for the reaction. It is a polar solvent that easily dissolves the precursors. J. H. Bang et al. in their review found that particle size was dependent upon alcohol concentration and alkyl chain length [42]. This is due to the fact the alcohols are absorbed on the surface of the metal nuclei, which can restrict growth of 
the metal nuclei. Hence, it is assumed that using ethanol would lead to truncated NP structures. To test this hypothesis, samples made using ethanol as a solvent were made and characterized, as seen in Figure 17. In Figure 17 (a), a cluster similar to Structure 1 is seen. Such clusters were found to be present throughout the sample, indicating the presence of $\mathrm{Pd} / \mathrm{Au}$ NPs. Both supported and unsupported clusters were observed and found to have the same characteristics. Here, an unsupported cluster was characterized for clarity.
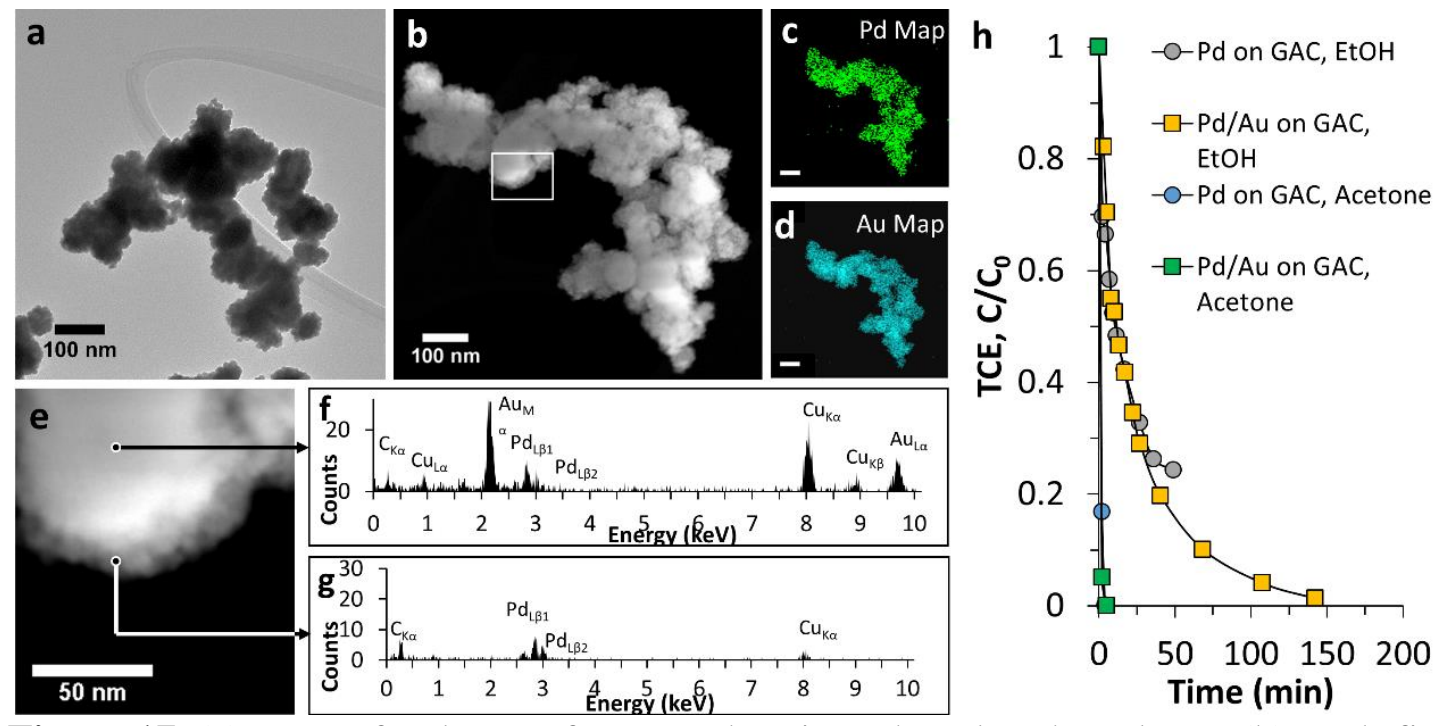

Figure 17. (a) TEM of a cluster of NPs made using ethanol as the solvent. (b) Dark-field STEM imaging of a cluster in the same cluster. A part of the cluster marked with the square was further examined in (e). EDS Maps (d) and (e) are of Pd and Au respectively. (e) A part of the cluster from (b) shows a less dense material covering a denser material. Extracted EDS spectrums from (f) the center and (g) edge show the Au and Pd peaks in the center and only Pd peaks at the edge, suggesting an Au core surrounded by a uniform Pd shell.

Seen in Figure 17 (a), the contrast between the metals is not as apparent as in the original Structure 1 in Figure 6 (a). The dark-field STEM image in Figure 17 (b), with the area marked by the box and enlarged in Figure 17 (e) at a higher magnification, provides some insight. The images indicate that the thickness of the shell was truncated, and that 
while this cluster has a core-shell design, the shell layer is so thin that it becomes hard to distinguish in the TEM image. This confirms the earlier hypothesis that ethanol restricts the growth of the metal nuclei. EDS maps in Figure 17 of (c) Pd and (d) Au confirm the composition of the structure. In Figure 17 (f) and (g), the extracted EDS spectrums from the center and edge of the cluster can be seen. The overall intensity is quite low due to the small size of the NPs and cluster area analyzed. In Figure 17 (f) from the center of the cluster, both Au and Pd peaks are observed with the Au intensity greater than Pd. In Figure $17(\mathrm{~g})$, the Au peak is absent and there is a small increase in the Pd peak (increased from 8 counts to 10 counts). The $\mathrm{Cu}$ peaks are from the grid, and the carbon is from the graphene support. The difference in the intensities of the Au and Pd peaks in these EDS spectrums suggest an aggregate-Pdshell $\mathrm{Au}_{\mathrm{Core}}$ configuration for this cluster as well. The TCE degradation results in Figure 17 (h), are in agreement with a reduced Pd-shell structure, indicating that samples formed in acetone that have a thicker Pd shell degrade TCE much faster than samples made in ethanol.

\section{Effect of Synthesis Time on Growth of Bimetallic NP Clusters}

In order to further study the interaction between the Pd/Au NPs, the synthesis time was changed to observe the formation and growth of the bimetallic clusters. The solvothermal process is mostly responsible for the growth of the NPs, and so this time was varied to understand how the NPs form and grow. Samples were observed at intervals of one-hour during synthesis, up to 24 hours. Here, the sample that was allowed to react for just 1 hour was evaluated, as it reveals significant findings, seen in Figure 18 and Figure19. 


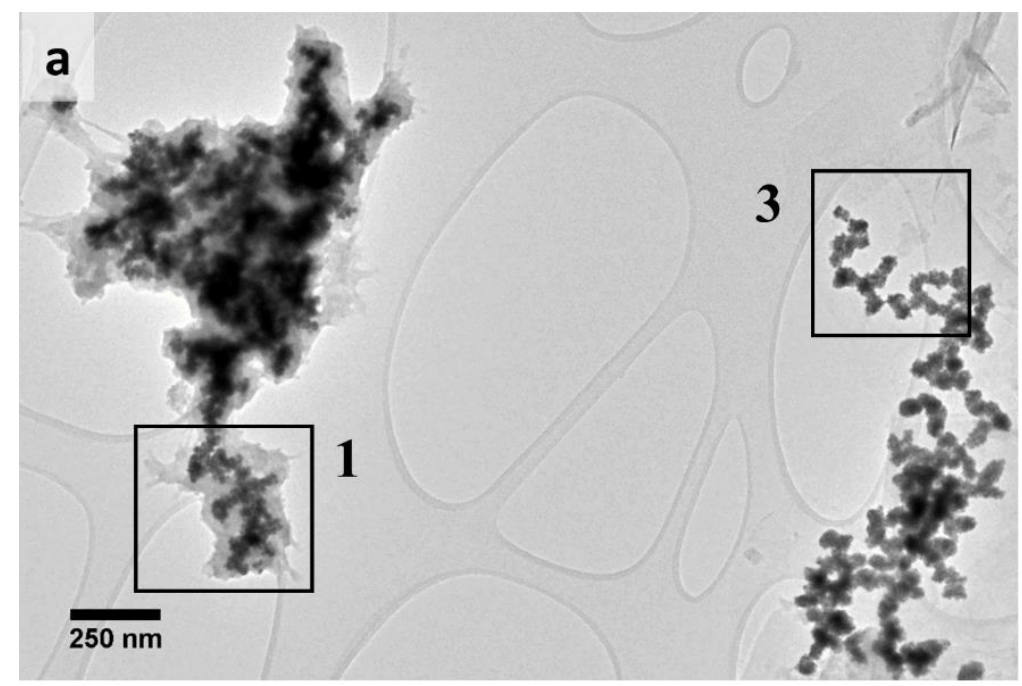

Structure 1

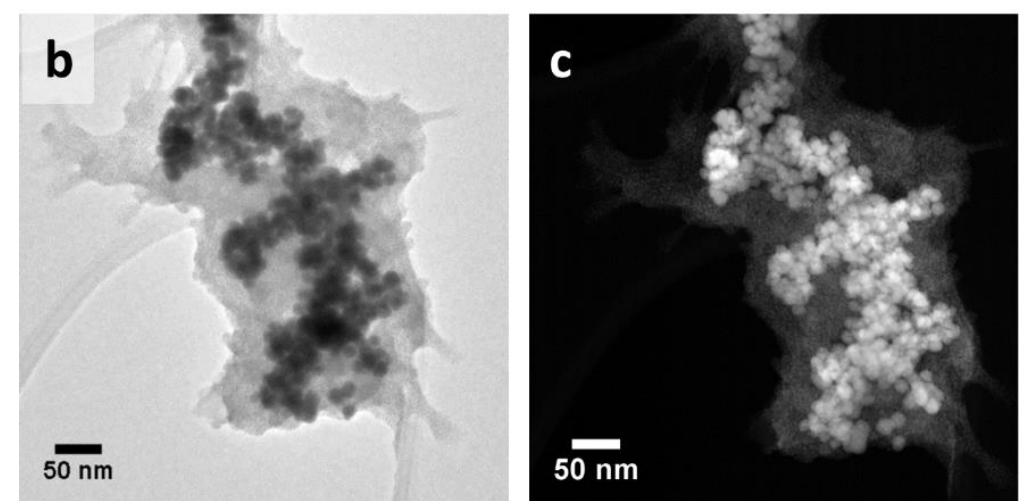

Figure 18. TEM image of a specimen with synthesis time controlled to 1 hour, allowing the observation of structure phenomena of an incomplete reaction. (a) Low magnification image showing different structures present on the same sample; areas marked by boxes, called Structure 1 and Structure 3, have been examined further. (b) Cluster strongly resembling Structure 1, (c) dark-field STEM image of this cluster confirming $\mathrm{Pd}_{\text {Shell }} \mathrm{Au}_{\text {Core }}$ formation. Structure 3 is examined in Figure 19.

In the relatively low magnification TEM image Figure 18 (a), two types of clusters were observed. The cluster to the left has a similar configuration to Structure 1, and further analysis shown in Figure 18 (b) and (c) confirmed this. Figure 18 (b) and (c) are brightfield TEM and dark-field STEM images, respectively, revealing the same core-shell structure previously observed. However, the Au core is more apparently made of smaller 
Au NPs congregated together. In the 24-hour sample, these cores appear to mesh together more as the smaller NPs lose their distinct outlines.
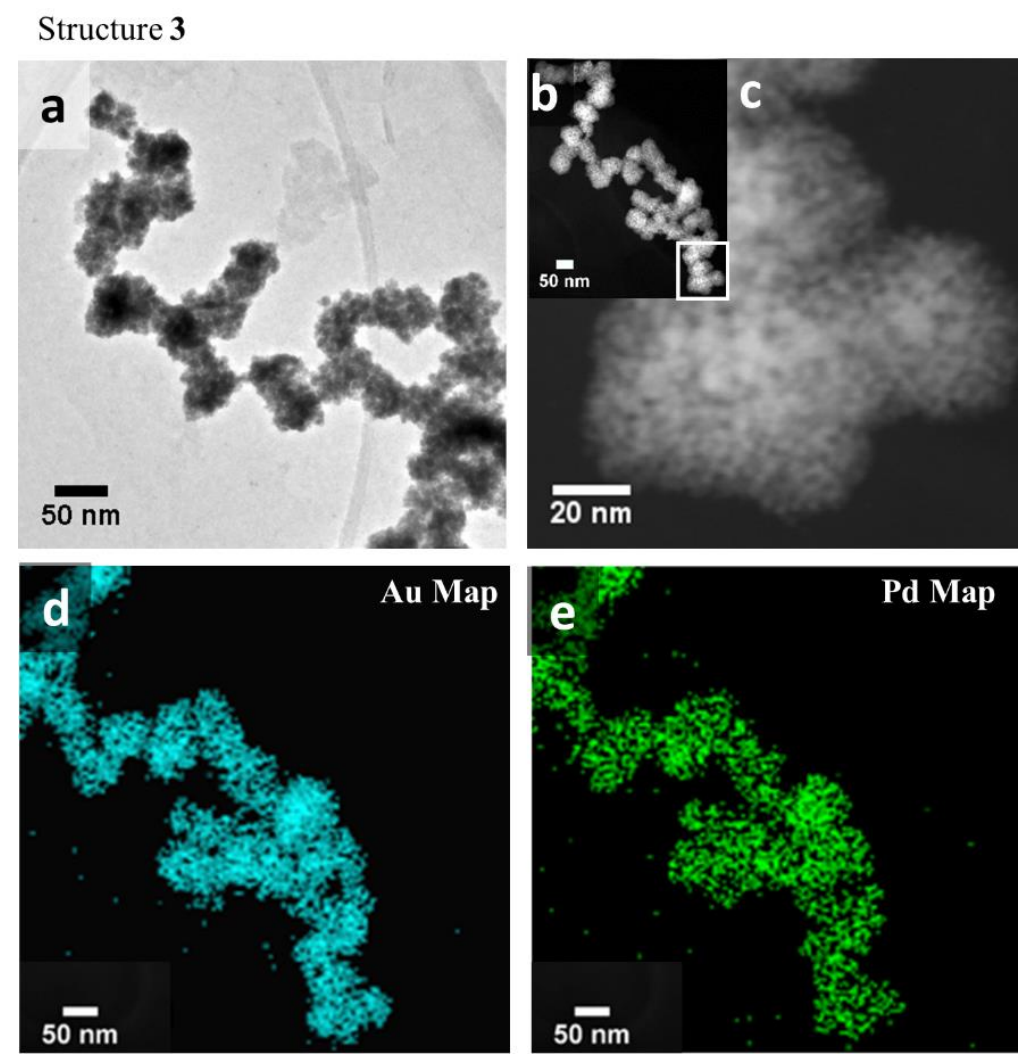

Figure 19. Images showing Structure 3. (a) TEM image and corresponding dark-field STEM image in inset (b). (c) A closer look at this cluster. EDS Maps of this cluster for (d) Au and (e) Pd.

The cluster to the right in Figure 18 (a), termed here as Structure 3, appears to have a different arrangement than Structure 1. This structure has been analyzed in Figure 19. Figure 19 (a) is a bright-field TEM image of Structure 3 cluster, while inset (b) is a darkfield STEM image of the same area rearranged slightly due to prolonged exposure to the beam. A closer look at an area in this cluster is seen in Figure 19 (c). This structure appears to be "unfinished." That is, the structure appears to be made of an agglomeration of NPs, 
but the clear core-shell configuration is absent. The EDS maps in Figure 19 (d) and (e) of $\mathrm{Au}$ and $\mathrm{Pd}$, respectively, suggest that the agglomerate is a mix of both metal NPs. The small size of the NPs proves challenging for EM techniques and requires advanced analysis, which is on-going. However, based on the analyses presented by past publications [60], it is assumed the NPs are of individual metals that are mixed together, which later grow into the core-shell configuration of Structure 1, seen in Figure 6.

The dark-field STEM images in Figure 18 (c) and Figure 19 (c) show a clear difference in the morphology of the two structures. It is important to note that Structure 3 is only present at lower synthesis times, suggesting that over time, Structure 3 becomes Structure 1. This means that sufficient time is needed for the reaction to be completed and for the final structures to form. Based on this finding, it is believed that the NPs are formed by first coming together to form unfinished clusters. As the NPs grow over time, a thick layer of agglomerated Pd NPs surround clusters of Au NPs.

\section{Effect of Synthesis Temperature on Growth of Bimetallic NP Clusters}

Another parameter that affects the formation of the clusters during the solvothermal process is the synthesis temperature. Since the sonication occurs in a water bath, the temperature of the forming solution is mostly controlled. However, an external temperature can be imposed on the material during solvothermal synthesis if the autoclaves are heated in an oven. Hence, the catalysts were synthesized at two temperatures, $70^{\circ} \mathrm{C}$ and room temperature $\left(22-25^{\circ} \mathrm{C}\right)$. 

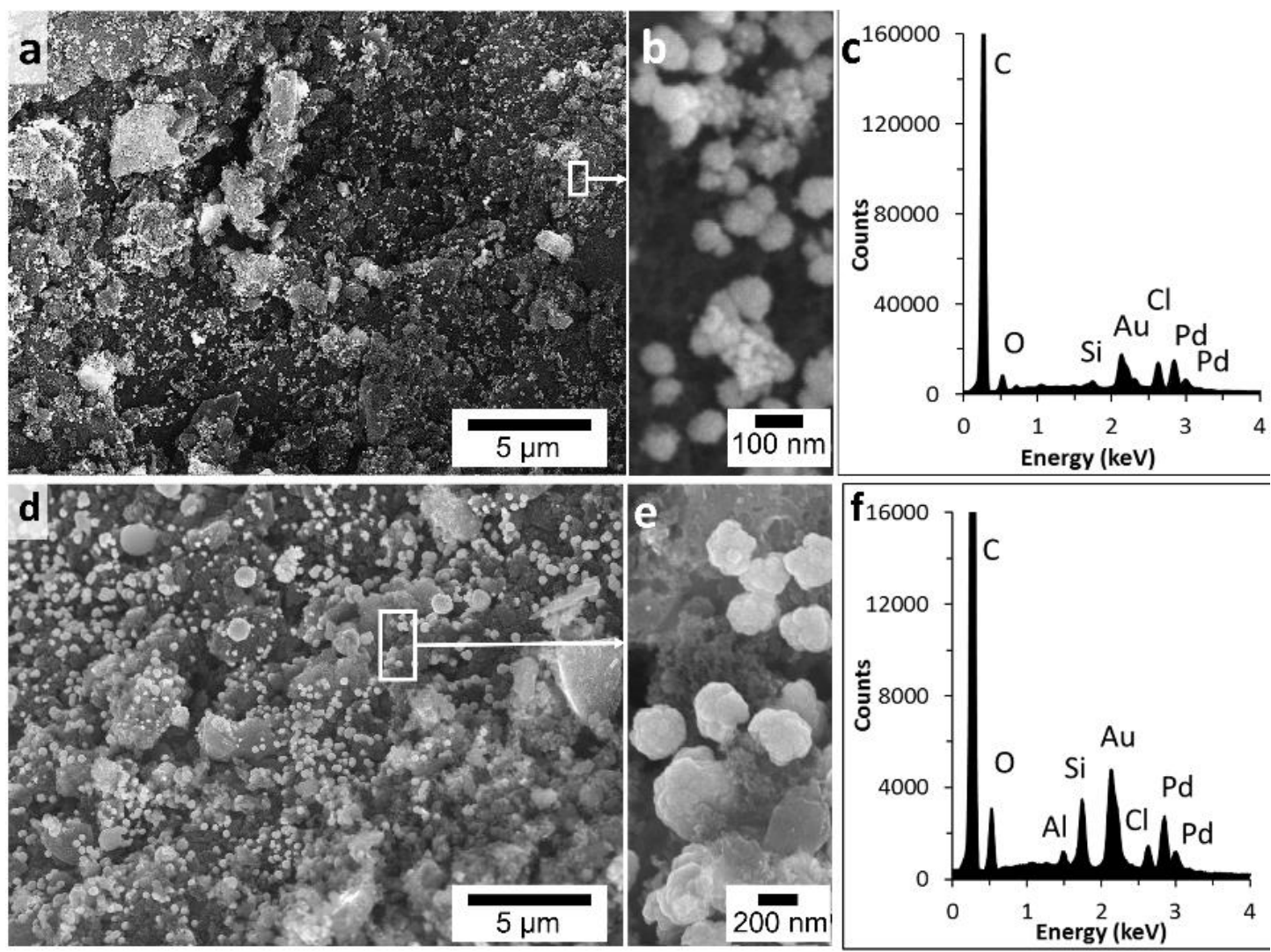

Figure 20. SEM images of (a) sample made at room temperature, and (b) at higher magnification, with (c) EDS spectrum showing NPs are made of Pd/Au. (d) sample made at $70^{\circ} \mathrm{C}$, and imaged at higher magnification, (e), with (f) EDS spectrum showing NPs are made of Pd/Au. The difference in size between the NPs made at the two synthesis temperatures is apparent.

These temperatures were chosen since $70^{\circ} \mathrm{C}$ is slightly hotter than the boiling point of acetone $\left(56^{\circ} \mathrm{C}\right)$, and room temperature $\left(22-25^{\circ} \mathrm{C}\right)$ is the most convenient temperature condition to maintain. These specimens were characterized as seen in Figure 20 (a) shows a survey of NPs uniformly distributed the surface of GAC synthesized at room temperature Figure 20 (b) shows the NPs cluster which are in the range of $20-50 \mathrm{~nm}$. Figure 20 (c) confirms the particles are composed of $\mathrm{Pd}$ and $\mathrm{Au}$. Chlorine $(\mathrm{Cl})$ and silicon $(\mathrm{Si})$ are contaminants of GAC introduced during handling. Figure 20 (d) shows the surface of the GAC-based catalyst synthesized at $70^{\circ} \mathrm{C}$, with Figure 20 (e) showing predominantly larger 
particles on the surface, in the range of $180-350 \mathrm{~nm}$. Figure 20 (f) confirms these NPs are composed of Pd and Au.

\section{Mechanism of Formation of NPs on the Carbon Support}

Based on the findings of this research, it is believed that the mechanism of material formation occurs as follows. First, the metallic precursors are added to a solvent, preferably acetone, and then sonicated. Initially, the precursors ionize, and during sonication, the sonolysis of water assists in the reduction of the metal ions to their native oxidative states. If Au is given a head start, the stable cores are formed first, followed by a thick, aggregate shell of Pd NPs. Different solvents can have an effect on the size of the aggregate shell and other properties of the material.

In the course of the solvothermal process, because of the attractive forces between Pd and Au, the tiny metal NPs simply agglomerate together. Once the carbon support is added, the NPs hybridize with the carbon surface and grow in size. Due to Pd's affinity to graphene, two types of structures are formed: Structure 1 are clusters of Pd and Au in an aggregate-PdShell $\mathrm{Au}_{\text {Core }}$ configuration, while Structure 2 are smaller Pd NPs dispersed through the surface of graphene. In fact, it is speculated that as Structure 2 is spread through the graphene support, it sometimes prevents Structure 1 -the clusters-from properly hybridizing with the carbon support. This leads to Structure 1 being either supported or unsupported. When the catalyst is tested against a contaminant, all unsupported clusters of Structure 2 are removed; however, here they were useful for unobstructed analysis. 


\subsubsection{Role of Carbon Support for Pd/Au Nanoparticle-Based Catalysts}

In the previous section, the formation of the NPs was studied in detail. In this section, the focus is on the effect of different carbon supports on the NP formation. An analysis of the $\mathrm{Pd} / \mathrm{Au}$ NPs on different carbon supports produced similar results, showing formation of Structure 1 and Structure 2 as seen in Figure 5 and Figure 6. In addition, the size of the individual, elemental NPs remained roughly the same on all carbon supports. However, other variations such as size and distribution of clusters over the carbon surfaces was found to vary. These results are examined here.

Carbon supports have often been used to support Pd and Au NPs, especially in the form of GAC [49], carbon black [61], and graphite [62]. These carbon-supported Pd/Au NPs were found to be favorable catalysts for removal of TCE via HDH [17], synthesis of hydrogen peroxide [49], electrooxidation of formic acid [61], etc. Four supports were studied here for their impact on optimization of catalyst activity for TCE removal: GAC, carbon black, graphite and graphite nanoplates. The term "graphite nanoplates" refers to graphite with a thickness of less than $100 \mathrm{~nm}$, following the definition previously outlined [63].

The difference between the graphene used previously in this thesis and the graphite nanoplates used in this section is in the procedure used to make the materials. For both materials, expandable graphite is sonicated for $15 \mathrm{~min}$ to from graphite nanoplates and 30 min for graphite nanoplates. For graphite nanoplates, this solution is allowed to rest for a shorter period of time and a larger amount of solution is removed to reclaim the material. This means the produced graphene are few layered, electron transparent and of relatively 
good quality; while the graphite nanoplates are much thicker, with low quality and some defects. While graphene has several good qualities as a support for Pd/Au NPs, graphite nanoplates are more cost effective. Since graphene was characterized in detail thus far as a support for Pd/Au NPs, graphite nanoplates are the focus in this part of the report.

These four supports have beneficial properties as supports for catalysts: both GAC and carbon black have adsorptive properties, while graphite and graphite nanoplates have more stable, ordered structures. The Pd/Au NPs were fabricated on the GAC, carbon black, graphite, and graphite nanoplates supports using identical procedures; however, a difference in the NP size and density was observed on each support. Pd/Au NPs on the four different carbon supports were characterized using the SEM, as seen in Figure 21. The arrows are pointing towards some NP clusters for ease in identifications.

In Figure 21 (a), (b) and (c), a GAC-supported Pd/Au NPs specimen were characterized, showing bimetallic NP clusters over the surface of GAC. Figure 21 (a) shows that the NP clusters are uniformly spread over the surface of the GAC and appear to be in the same size range. The GAC surface was further examined in Figure 21 (b) at a higher magnification showing the NPs appear to have an irregular morphology. At the highest magnification, seen in Figure 21 (c), the clusters look similar to those seen in Figure 5 (a). 

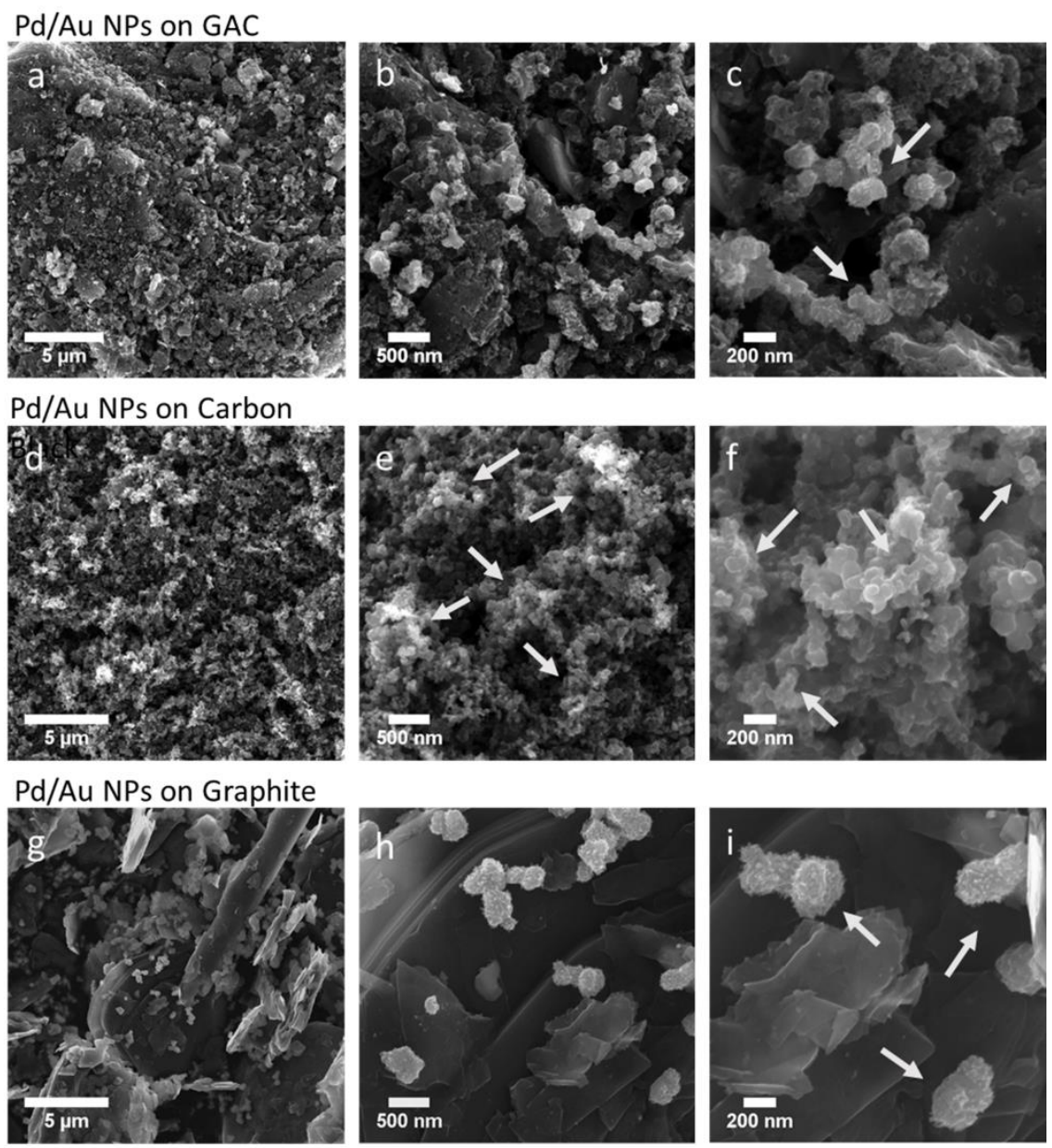

$\mathrm{Pd} / \mathrm{Au}$ NPs on Graphite Nanoplates
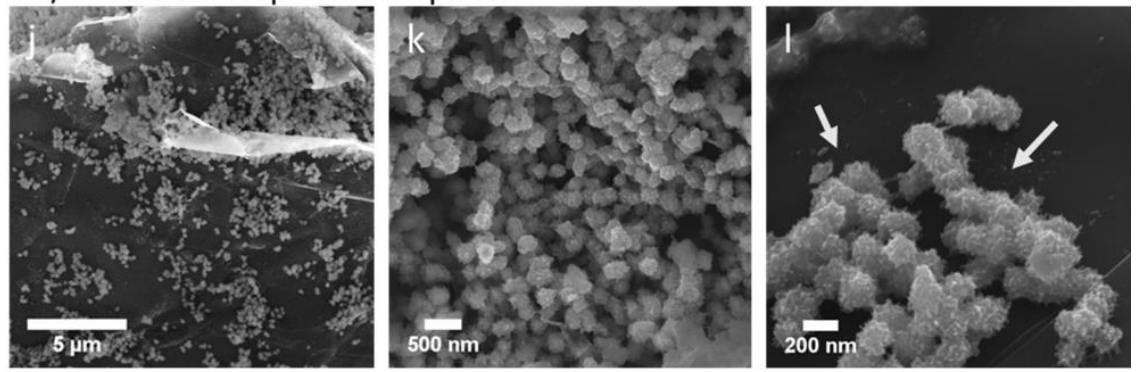

Figure 21. SEM images of $\mathrm{Pd} / \mathrm{Au}$ NPs on various carbon supports. From left to right, images increase in magnification. $(\mathrm{a}-\mathrm{c})$ Images of GAC-supported $\mathrm{Pd} / \mathrm{Au} \mathrm{NPs}$, showing (a) dispersion, (b) size, and (c) topography of these NPs. (d - f) are images of carbonblack supported Pd/Au NPs, showing (d) wider distribution of NPs and (e) compactness of the NPs, (f) seen at higher magnifications. $(g-h)$ are images of graphite-Pd/Au NPs showing (g) poor distribution of NPs over the surface of support, and (h) relatively larger size of the NPs and (i) at a higher magnification. Images $(\mathrm{j}-\mathrm{k})$ are of the $\mathrm{Pd} / \mathrm{Au} \mathrm{NPs}$ on graphite nanoplates showing (j) large number of NPs over the surface, $(\mathrm{k})$ relatively larger size and (1) the morphology of the NPs. Arrows are provided to help with identification of NP clusters. 
The carbon black supported Pd/A NPs was studied next in Figure 21 (d), (e), and (f). Seen in Figure 21 (d) the NP clusters are hard to identify due to the highly irregular and small size of the support. At higher magnifications, seen in Figure 21 (e), the NP clusters become slightly more apparent because of the difference in contrast, as pointed out by the arrows. In Figure 21 (f), one such cluster is observed. This cluster also appears to be similar those seen in Figure 5 (a).

Next, the graphite supported Pd/Au NPs was examined in Figure 21 (g), (h) and (i). Figure 21 (g) shows that unlike the GAC and carbon black, here, the clusters are sparsely distributed over the surface of graphite. In addition, larger clusters appear to be formed that the previous two specimens, as seen in Figure $21(\mathrm{~h})$. The clusters are further examined in Figure 21 (i), showing the same uneven morphology.

Finally, in Figure 21 (j), (k) and (1), the graphite nanoplates supported Pd/Au NPs was observed. In Figure 21 (j), the clusters are well distributed over the surface of the graphite nanoplates. A closer look at Figure 21 (k) shows that the NPs appear to be closer together, though of a medium size. In Figure 21 (1), the same irregular morphology of the NPs observed.

The range and average size of the NPs are presented in Table 3. The Pd/Au NPs on GAC have clusters in the range of $100-200 \mathrm{~nm}$, with an average size of $146.73 \pm 35 \mathrm{~nm}$, while the Pd/Au NPs on carbon black have clusters in the range of $50-150 \mathrm{~nm}$ with an average size of $90.08 \pm 53 \mathrm{~nm}$. The $\mathrm{Pd} / \mathrm{Au}$ NPs on graphite have clusters in the range of $200-350 \mathrm{~nm}$ with an average size of $273.57 \pm 50 \mathrm{~nm}$, and the $\mathrm{Pd} / \mathrm{Au}$ on graphite nanoplates have clusters in the range of $150-250 \mathrm{~nm}$ with an average size of $203.37 \pm 42 \mathrm{~nm}$. These 
observations suggest that smaller NP and clusters are formed on GAC and carbon black, while larger sized NPs form on graphite and graphite nanoplates

Table 3. Range and Average size of the NP clusters for the various materials

\begin{tabular}{llcc}
\hline$\#$ & Material & $\begin{array}{c}\text { Range of NP Cluster } \\
\text { Sizes }\end{array}$ & $\begin{array}{c}\text { Average Size of NP } \\
\text { Clusters }\end{array}$ \\
\hline $\mathbf{1}$ & $\mathrm{Pd} / \mathrm{Au}$ NPs on GAC & $100-200 \mathrm{~nm}$ & $146.73 \pm 36 \mathrm{~nm}$ \\
$\mathbf{2}$ & $\mathrm{Pd} / \mathrm{Au}$ NPs on carbon black & $50-150 \mathrm{~nm}$ & $90.078 \pm 54 \mathrm{~nm}$ \\
$\mathbf{3}$ & $\mathrm{Pd} / \mathrm{Au}$ NPs on graphite & $200-350 \mathrm{~nm}$ & $273.57 \pm 50 \mathrm{~nm}$ \\
$\mathbf{4}$ & $\mathrm{Pd} / \mathrm{Au}$ NPs on graphite nanoplates & $150-250 \mathrm{~nm}$ & $203.37 \pm 42 . \mathrm{nm}$ \\
\hline
\end{tabular}

It is clear that each of the supports appears to interact differently with the metal NPs, which causes a variation in the sizes. One reason for this could be due to the surface of each support—-both carbon black and GAC have disordered structures, while the graphite and graphite nanoplates have more of an ordered, layer-by-layer structure that allow the NPs to deposit at specific sites and grow uniformly. Moreover, both GAC and carbon black are highly adsorptive, which possibly disperses the metal NPs more across the surface and prevents larger aggregations from occurring. This phenomenon is presently under further investigation using more advanced techniques.

Next, the uptake, or retention of the NPs, by each support during synthesis was quantified here as the mass concentration of NPs actually hybridized to each carbon support. Previously, UV-vis spectroscopy has been shown to successfully calculate the uptake of $\mathrm{Au}$ NPs on $\mathrm{Al}_{2} \mathrm{O}_{3}, \mathrm{MgO}$, and $\mathrm{SiO}_{2}$ supports [53]. Hence, the same method was adopted here and modified to calculate the uptake of the NPs by the different carbon supports. In addition, each carbon material and the respective supported catalyst was tested 
against model contaminant TCE. This uptake results and decomposition of TCE is shown in Figure 22.

Based on the calibrations and theoretical calculations, the maximum concentration of Pd NPs and Au NPs available for uptake is $1.42 \mathrm{mg} / \mathrm{L}$ and $65.21 \mathrm{mg} / \mathrm{L}$, respectively. All supports have an uptake of NPs of at least $88 \%$ or more of this maximum concentration. Seen in Figure 22 (a), GAC and carbon black have similar uptakes of Pd NPs, only leaving behind $0.07 \mathrm{mg} / \mathrm{L}$ and $0.06 \mathrm{mg} / \mathrm{L}$, respectively. Next, graphite nanoplates appear to leave $0.1 \mathrm{mg} / \mathrm{L}$ of Pd NPs, suggesting that despite the lack of adsorptive qualities of GAC and carbon black, the large surface area provides a platform for the NPs to deposit. Finally, graphite had the lowest uptake, leaving behind $0.16 \mathrm{mg} / \mathrm{L}$ of Pd NPs. This trend was also observed for uptake of Au NPs, seen in Figure 22 (b), with GAC and carbon black leaving behind $3.38 \mathrm{mg} / \mathrm{L}$ and $2.86 \mathrm{mg} / \mathrm{L}$ of $\mathrm{Au} \mathrm{NPs}$, followed by graphite nanoplates leaving behind $4.51 \mathrm{mg} / \mathrm{L}$ of Au NPs, and graphite with $7.34 \mathrm{mg} / \mathrm{L}$.

While some variations in uptake in NPs was observed, the final concentrations of bimetallic NPs on the carbon supports are not significantly different. However, the sizes of the NPs and clusters on the supports are significantly different. Specifically, while graphite nanoplates have relatively similar uptake of NPs as GAC and carbon black, the NPs deposit as larger clusters on graphite nanoplates. Hence, it is believed the extended surface area of graphite nanoplates improves the uptake of NPs and therefore the HDH removal of TCE, as seen in Figure 22 (c) and (d).

Seen in Figure 22 (c), first, the bare carbon materials were tested with TCE as-is. GAC appeared to have fastest removal, adsorbing close to $40 \%$ of TCE within the first ten min. 
This was closely followed by carbon black, which also adsorbed TCE, but at a slightly slower rate, removing $30 \%$ of TCE within the first ten mins. As expected, both the graphite nanoplates and graphite did little to remove TCE over time.
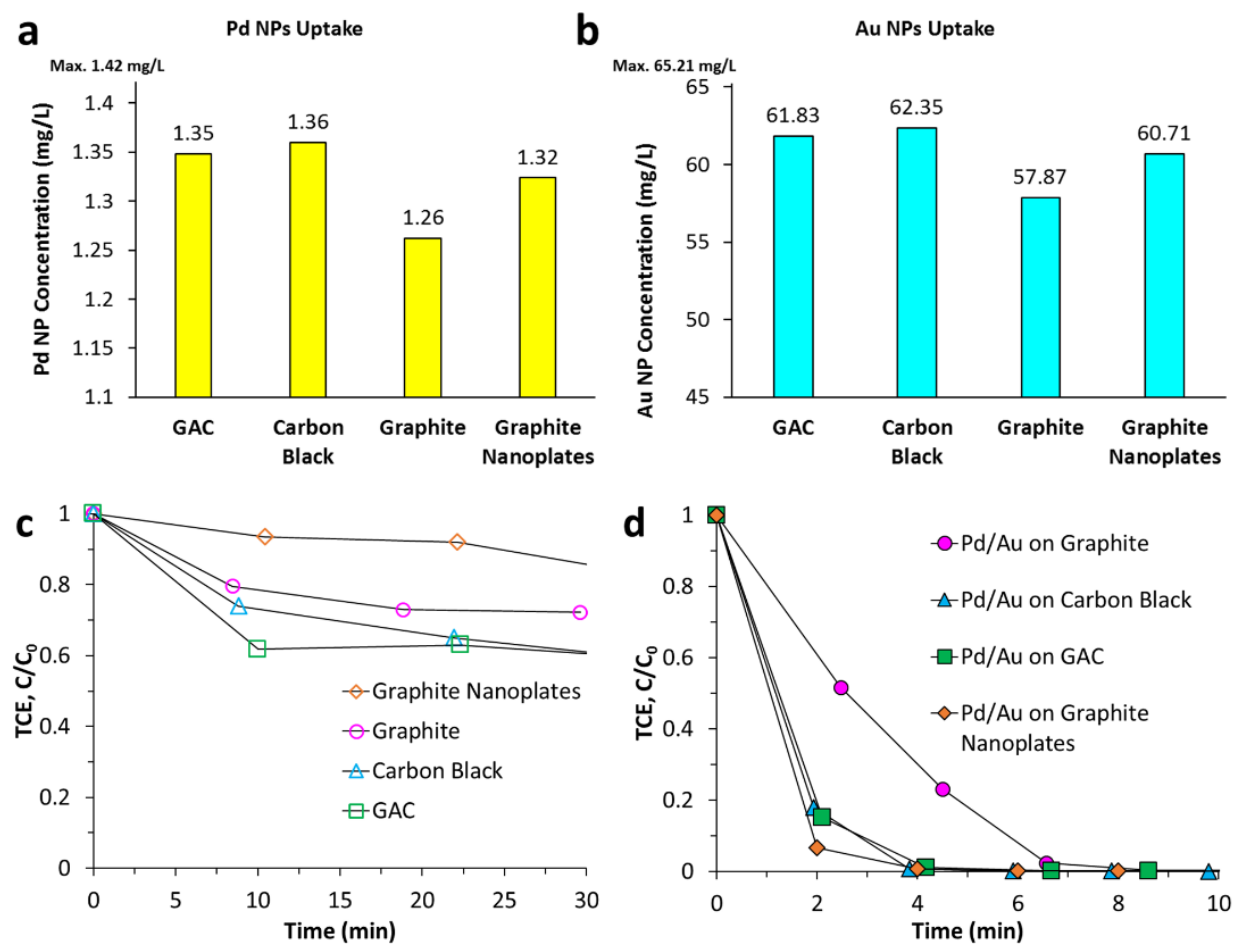

Figure 22. Retention of (a) Pd NP and (b) Au NP on the GAC, carbon black, graphite and graphite nanoplates supports during synthesis. (c) The decomposition of TCE over time, plotted as normalized concentration of TCE over time, in the presence of just carbon supports and (d) Pd/Au NPs hybridized to the carbon supports.

Next, Pd/Au NPs on the various carbon supports were tested against TCE as seen in Figure 22 (d). The presence of the Pd/Au NPs caused the reactions to occur much faster. Surprisingly, the Pd/Au NPs on graphite nanoplates, Pd/Au NPs on carbon black, and $\mathrm{Pd} / \mathrm{Au}$ NPs on GAC performed roughly the same, followed by the Pd/Au NPs on graphite catalyst. When fit into equation (1), the Pd/Au NPs on GAC samples gave a $k_{o b s}$ of 1.10 $\min ^{-1}$, while Pd/Au NPs on carbon black had a $k_{o b s}$ value of $1.13 \mathrm{~min}^{-1}$, and $\mathrm{Pd} / \mathrm{Au} \mathrm{NPs}$ on graphite nanoplates produced a $k_{o b s}$ of $0.94 \mathrm{~min}^{-1}$. Finally, the Pd/Au NPs on graphite 
catalysts gave a $k_{o b s}$ of $0.54 \mathrm{~min}^{-1}$. This $\mathrm{k}$ value for the $\mathrm{Pd} / \mathrm{Au}$ NPs on GAC is consistent with rates observed in Section 2.4.6.

It is important to note that the bare carbon supports differ from each other in surface morphology, which, in part, is responsible for the difference the in number of NPs formed on each support and the sizes of the clusters on the supports. The particle size and shape of each support is different, which has a direct impact on the catalytic HDH of TCE. A larger available surface area allows more of the TCE to interact with the catalyst and therefore $\mathrm{HDH}$ will occur faster. Among the four types of carbon-supported Pd/Au NPs, the Pd/Au NPs on graphite catalyst had the lowest surface area among the carbon supports, and the NPs hybridized to this support grew to the largest sizes, causing an overall decrease in available surface area, and hence, interaction with the TCE.

In contrast, while the NP size were relatively lower for the $\mathrm{Pd} / \mathrm{Au}$ NPs on graphite nanoplate catalysts, the graphene-like properties of the large surface area of the support allow more NPs to deposit. Hence, the $k_{o b s}$ of $\mathrm{Pd} / \mathrm{Au}$ NPs on graphite nanoplates was much larger than that of Pd/Au NPs on graphite. In the case of the Pd/Au NPs on carbon black and $\mathrm{Pd} / \mathrm{Au}$ NPs on GAC, both of these carbon supports exhibit adsorption and have smaller NPs hybridized to the surfaces. This allows faster rates of degradation. Despite carbon black and GAC having different structures [64], their adsorptive nature, combined with the dispersion and size of the metallic NPs, causes them to behave in a similar manner.

However, it is also important to take the particle size into consideration for available surface area of these supports. The carbon black in this study has a particle size of $50 \mathrm{~nm}$, used for consistency with other reports [65]-[67], while the GAC has a particle size closer 
to $70 \mu \mathrm{m}$. Hence, the carbon black used here has a much larger surface area than the GAC.

Despite this, the $\mathrm{Pd} / \mathrm{Au}$ NPs on carbon black catalyst produces the same $k_{\text {obs }}$ as the $\mathrm{Pd} / \mathrm{Au}$ NPs on GAC, suggesting that when normalized for surface area, the rate of decomposition of TCE for the Pd/Au NPs on GAC catalyst would be much higher. Thus, GAC is considered an ideal support for the Pd/Au NPs

Pre-treatment of GAC as a support for Pd/Au NPs

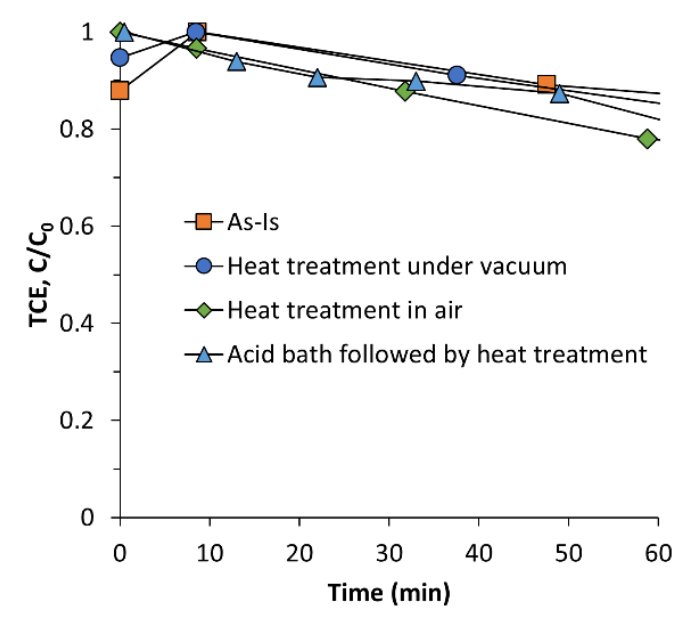

To ensure the adsorptive activity of GAC
was maximum, the as-purchased GAC was
treated using 3 different treatments: heating
under vacuum to $250^{\circ} \mathrm{C}$ for 1 hour, heating in
air to $500^{\circ} \mathrm{C}$ for 1 hour and immersing in acid
bath of $2 \mathrm{M} \mathrm{HCl}$ for 6 hours followed
thorough wash in DI water and dried under

Figure 23. Adsorption of TCE with GAC received as-is, with heating under vacuum and in air, and finally after an acid bath. vacuum at $100^{\circ} \mathrm{C}$ for 1 hour. The GAC retrieved from these processes were compared against the as-purchased GAC, for their ability to adsorb TCE. As seen in Figure 23 , the different treatments did not have an effect on the GAC's adsorptive performance. Hence, the GAC in this project was used as-purchased for the synthesis of all GAC-based catalysts.

\subsection{Conclusions and Future Work}

A new catalyst material was developed for the removal of TCE by combining the large surface area and adsorption of carbon supports with the chemical decomposition of 
Pd/Au NPs. A simple, environmentally friendly process was developed to synthesize these catalysts, which were found to be more efficient at TCE removal than corresponding commercial catalysts. These catalysts can be used for range of concentrations, over several cycles of high doses of contaminations and have tunable rate of reactions. This optimization was achieved by studying the formation of NPs during synthesis, under different conditions. Here, TCE was the model contaminant for which the catalyst material was optimized, however preliminary results (not included here) show this catalyst is equally effective against similar contaminants like TCP and CT.

In future work, the catalyst can be further optimized similar chlorinated ethenes, and to handle multiple VOCs at once. This catalyst has proven to be useful for HDH and has potential against other types of degradation mechanism such as hydrodeoxygenation and N-N hydrogenolysis. These results will be more conclusive when tested in flow reactors, which is a step towards scaling-up of this green synthesis for large-scale applications. 


\section{Carbon-Supported $\mathrm{Fe}_{3} \mathrm{O}_{4} \mathrm{NPs}$}

\subsection{Current Literature: Status and Limitations}

In this section, the design and synthesis, along with the characterization, and electrochemical and magnetic testing results of carbon-supported $\mathrm{Fe}_{3} \mathrm{O}_{4} \mathrm{NPs}$ material system are discussed. Although $\mathrm{Fe}_{3} \mathrm{O}_{4}$ is also used for catalytic removal of $p$-nitrophenol in water [6], here graphene supported $\mathrm{Fe}_{3} \mathrm{O}_{4} \mathrm{NPs}$ were specifically developed for electromagnetic applications. At the time of writing this thesis, some of the results presented here are under review for publication.

One of the more interesting properties exhibited by TMs is magnetism. Several TMbased substances exhibit magnetic properties such as ferromagnetism, ferrimagnetism and antiferromagnetism. While these magnetic properties are well known, it would be helpful to revisit the definitions of these terms.
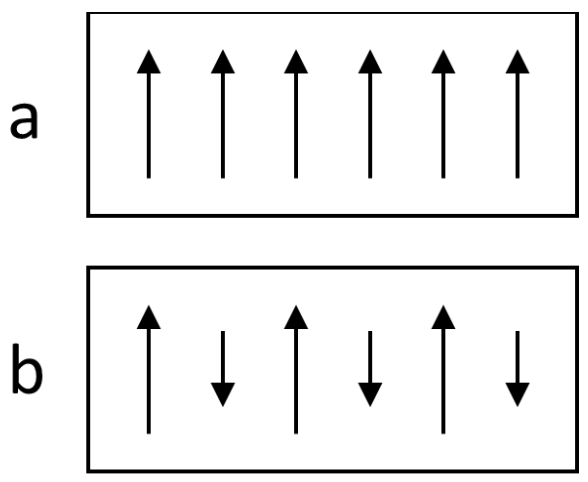

C

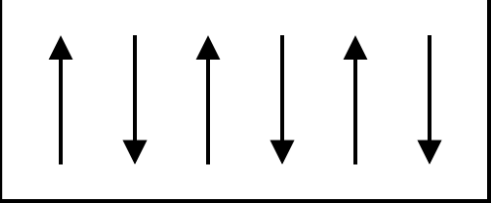

Figure 24. Electron spin directions in (a) ferromagnetic,

(b) ferrimagnetic and

(c) antiferromagnetic materials.
The electron spin directions for ferromagnetism, ferrimagnetism and antiferromagnetism is schematically represented in Figure 24. Ferromagnetism, represented in Figure 24 (a), is exhibited by materials when the spins of the electrons are all aligned in parallel and in the same direction. Ferrimagnetism, Figure 24 (b), is exhibited when the material has populations with opposing magnetic moments, however due to unequal moments within the 
material, a net magnetic moment is produced. Antiferromagnetic materials, Figure 24 (c), are similar to ferromagnetic materials; that is these materials also often tend to have populations with opposing magnetic moments. When these opposing moments are equal, there is no net magnetic moment, which is called antiferromagnetism.

The three magnetic properties of ferromagnetism, ferrimagnetism and antiferromagnetism are independent of external magnetic fields. However, magnetism isn't necessarily an inherent property of a material, for example, in certain materials, the spins are randomly aligned until they are exposed to a strong magnetic field. This external field causes the spins to rearrange themselves in an orderly manner, causing ferromagnetic properties, which are retained even after the external field is removed. Such materials are called permanent magnets. In addition to the three magnetic properties that have been introduced above, there are other magnetic properties such as diamagnetism and paramagnetism, in which the external magnetic field generates a net magnetic moment within the material. As these properties are not directly related to the research presented here, they have not been discussed.

Some materials can be manipulated so that their magnetic properties can be changed from one state to the other, usually induced by electric current. The ability to do so has found noteworthy applications, such as in spintronics, electromagnets, AC transformers, magnetic shields and for information storage [11]. Most of these applications, including non-volatile memory for storing data in hard disk drives, solid state drives, floppy disks, and magnetic tapes, etc. uses magnetic fields generated by current-induced electromagnets. The biggest drawback of current-induced magnetic fields is the amount of power needed 
to achieve this magnetic switching [68]. A lot of this power is lost through Joule heating, which can lead to increase in temperature of the material. Therefore, this method for magnetic switching has poor efficiency [11].

In 2003, D. Chiba et al were the first to report electric-field control of coercivity and electric-field-assisted magnetization reversal in magnetic materials [69]. Since then, there has been growing interest in the idea of using electric fields to control the magnetism of a material. In theory, an electric-field would only need a sufficiently supply of charge to induce the change in magnetic moment of the material. The system would be contained in an electrical cell, where this charge would be utilized in the charge/discharge of the cell, while the strength and direction of the electric field should be enough to affect the material located in the electric field. This set-up would reduce the energy dissipation by a factor of nearly 100 , making the technology comparable to present semiconductor field-effect transistors [11]. This ability to influence in magnetic properties by exposure to electric fields is due to an intrinsic property of the material, called the ME effect. The ME effect is a property of the material which denotes the coupling effect between the magnetic and the electric properties of a material.

A complete, reversible control of magnetic moment has not yet been achieved. Partial reversible variations have been seen in the past with materials such as $\gamma-\mathrm{Fe}_{2} \mathrm{O}_{3}$ [7], [8], $\mathrm{MnFe}_{2} \mathrm{O}_{4}$ [8], $\mathrm{ZnFe}_{2} \mathrm{O}_{4}$ and $\mathrm{CuFe}_{2} \mathrm{O}_{4}$ [9] when tested in electric fields generated by lithium ion batteries (LIBs). More recently, perovskite crystal structures like $\mathrm{La}_{1-\mathrm{x}} \mathrm{Sr}_{\mathrm{x}} \mathrm{MnO}_{3}$ [70] and $\mathrm{La}_{1-\mathrm{x}} \mathrm{Sr}_{\mathrm{x}} \mathrm{CoO}_{3}$ [71], [72] have also been found to exhibit some degree of reversibility in electric field generated by hybrid SCs and transistors respectively. 
Recently, collaborators Dr. Guodong Wei and Dr. Yanxue Chen at Shandong University, China, have shown a reversible control of magnetism in $\mathrm{Fe}_{3} \mathrm{O}_{4} \mathrm{NPs}$, when exposed to electric fields of LIB [10], which has never been reported before. In this publication, the observable change magnetic moment was found to be $1 \%$, switching from $70.2 \mathrm{emu} / \mathrm{g}$ to $69.5 \mathrm{emu} / \mathrm{g}$ and back. Some reasons for this low magnetic reversibility are attributed to the material used: commercially purchased $\mathrm{Fe}_{3} \mathrm{O}_{4} \mathrm{NPs}$ were used bound to carbon black for increased conductivity. In addition, testing with a LIB is often limited by the high mobility of the Li ions that can cause permanent damage to the materials and are also unsafe to use. While the published results are exiting and novel, noting the ability to reversibly control magnetic properties of $\mathrm{Fe}_{3} \mathrm{O}_{4} \mathrm{NPs}$, here, the goal was to achieve more significant change in magnetic moment with careful design of material and by improving the testing conditions.

\subsection{Design of Material and Electrode for Control of Magnetism in an Electric Field}

For the purpose of reversibly testing the magnetic moment of a material in electric fields of electrochemical cells, the materials are often designed to be integrated into the electrode. The conventional electrode, conceptualized in Figure 25 (a), is usually made up of 4 components: active material which plays the main role in the electrochemical cell, carbon used to increase conductivity, a binder such as polyvinylidene fluoride (PVDF) and current collector, usually a metal like copper, aluminum or nickel. In this project, $\mathrm{Fe}_{3} \mathrm{O}_{4}$ NPs play the role of active material. PVDF is an adhesive polymer that binds the active material and carbon to the current collector. The ratios of the components vary depending on the materials and the type of electrochemical cell. 
While $\mathrm{Fe}_{3} \mathrm{O}_{4}$ NPs have been tested as electrodes for switching magnetic properties in LIB in the past, they have always been used with some form of a carbon such as graphite [7] and acetylene black [10] (as electronically conducting additives) in ratios up to $10 \%$. Graphene has several advantages over the conventional carbon additives as it has high surface area and excellent electrical properties, which makes it highly desirable as an electrode component [73]-[75]. In fact, while graphene has often been used as a component in electrodes for both LIBs and SCs [73]-[76], it is yet to be implemented in electrodes when testing magnetic properties.

a

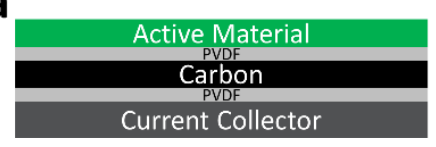

b

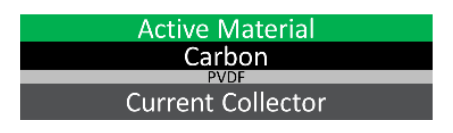

C

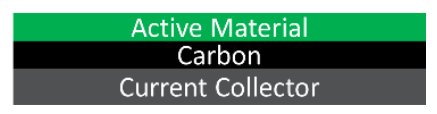

Figure 25. Schematics of (a) conventional electrodes, (b) electrode design used in this project and (c) eventual goal of the project.

To increase conductivity in the electrode, here, the design of the $\mathrm{Fe}_{3} \mathrm{O}_{4} \mathrm{NPs}$ was to directly hybridize the NPs onto the carbon support, as seen in Figure 25 (b). This alleviated the need for a binder between the active material and carbon, thus improving the flow of electrons in the electrode. A binder was still needed to bind the graphene supported NPs to a metal conductor; however, the overall conductivity of the electrode increased since the transmission of electricity was better, and hence the electrode had longevity over several cycles. The eventual goal of this project, seen in Figure 25 (c), is to get rid of PVDF completely, by growing the graphene on the metal foil and directly depositing NPs on this graphene. Although this is one of the goals of the project, it is out of the scope for this thesis and the work presented here. 
This modified electrode was designed to be versatile enough to be tested in both a LIB and SC. As this project was a collaboration with Shandong University, that had an established set-up for testing control of magnetism in LIB, the material synthesis and characterization was carried out at Portland State University, while the electrical and magnetic testing was carried out at Shandong University. However, the experiments with SC took place at Portland State University, where a SC was built, optimized and used for electrochemical testing of this project. The magnetic testing for the SC was carried in a vibrating sample magnetometer (VSM) at the Applied Magnetics Laboratory, Oregon State University.

\begin{tabular}{|l|}
\hline \multicolumn{1}{|c|}{ I. Material Design and } \\
Synthesis \\
Active Material: \\
- $\mathrm{Fe}_{3} \mathrm{O}_{4} \mathrm{NPs}(\sim 10 \mathrm{~nm})$ \\
- Spinel Crystal Structure \\
Synthesis Process: \\
- Graphene: Liquid \\
- Nxfoliation and CVD \\
SPs: Solvothermal \\
Synthesis \\
Material characterization \\
- SEM/TEM/XRD
\end{tabular}

\begin{tabular}{|l|}
\hline II. Electrode Fabrication \\
Electrode Design: \\
- Current collector \\
- Counter electrode \\
Binder: \\
- Polyvinylidene fluoride \\
- $\mathrm{PVDF}$ No Binder \\
Active Material Slurry: \\
- Fe $\mathrm{O}_{4} \mathrm{NPs}$, carbon \\
black/graphene and \\
PVDF on Cu foil
\end{tabular}

\begin{tabular}{|l|}
\hline III. Electrochemical Testing \\
Testing set-up: \\
- Electrochemical cell \\
setup: LIB and SC \\
- Electrolyte and \\
separator \\
- Voltage range, 100-0.1 \\
mV/s \\
- Control: Commercial \\
NPs on carbon \\
Testing: \\
- Cyclic Voltammetry \\
- Charge/discharge
\end{tabular}

IV. Magnetic Testing
Magnetic Testing:
- In-situ
charge/discharge in
SQUID (LIB)
- In-situ
charge/discharge in
VSM (SC)
Feedback Loop:
- Analyze data and
results
- Optimize NPs and
testing accordingly.

Figure 26. Detailed aspects of the carbon-supported $\mathrm{Fe}_{3} \mathrm{O}_{4}$ NPs material synthesis, electrode fabrication, electrochemical testing and magnetic testing.

Building on the considerations from the previous project, four different aspects of the project were studied here, as listed in Figure 26. In this project, focus was on design and development of a material which can exhibit a reversible control of magnetism in an electric field. Following this, some attention was spent on uniform electrode fabrication, electrochemical setup and finally, the magnetic testing. 


\subsection{Materials and Methods Used}

\subsubsection{Synthesis of Carbon-Supported $\mathrm{Fe}_{3} \mathrm{O}_{4}$ Nanoparticles}

Synthesis of $\mathrm{Fe}_{3} \mathrm{O}_{4} \mathrm{NPs}$ on graphene

To make $89 \mathrm{wt} \%$ of active material on carbon support, $45 \mathrm{mg}$ iron (III) acetylacetonate, $\mathrm{Fe}\left(\mathrm{C}_{5} \mathrm{H}_{7} \mathrm{O}_{2}\right)_{3}$, (97\%, Sigma-Aldrich Catalog\# F300) was added to $20 \mathrm{~mL}$ absolute ethanol (200 proof). $1.2 \mathrm{mg}$ of the carbon support (usually graphene) was added and the solution was magnetically stirred for $30 \mathrm{~min}$. The graphene used here were the same as those used in the previous project, as described in Section 2.3.1 and Section 2.4.1. The mixture of precursor, solvent and graphene was transferred to a Teflon-liner in a stainless-steel autoclave and closed. This autoclave was then moved into a preheated oven at $180^{\circ} \mathrm{C}$. No external gas was introduced, and the pressure was autogenous. No further agitation, stirring or mixing was introduced. After 24 hours, the autoclave was removed and allowed to cool using forced air cooling for an hour. At this point, the autoclave was cool enough to be handled and any built-up internal pressure reached atmospheric pressure. Once the autoclaves were opened, the samples were filtered and washed by centrifugation at 14,000 rpm for 15 min with fresh solvent and allowed to dry.

\section{Electrode Fabrication}

To make the electrode, a ratio of $90 \mathrm{wt} \% \mathrm{Fe}_{3} \mathrm{O}_{4} \mathrm{NPs}$ on graphene material and $10 \%$ PVDF binder was used. Since PVDF is usually in powder form, the $\mathrm{Fe}_{3} \mathrm{O}_{4} \mathrm{NPs}$ on graphene material and PVDF were initially mixed together, after which 10wt\% NMP was added and sonicated for $10 \mathrm{~min}$. This created a thick paste which was then evenly spread over the metal conductor. Control samples were made by combining $80 \mathrm{wt} \%$ of commercial $\mathrm{Fe}_{3} \mathrm{O}_{4}$ 
NPs (Alibaba, China) and 10wt\% of either carbon black (Fuel Cell Store, item \#590106) or graphene, with 10wt\% PVDF using NMP.

These slurries were uniformly spread over Cu foil (dimensions $1 \mathrm{~cm} \mathrm{X} 1 \mathrm{~cm} \mathrm{X} 20 \mu \mathrm{m}$ ) and dried at chamber electric furnace at $70^{\circ} \mathrm{C}$ for 10 hours to form a working electrode. Generally, electrodes were made with weight of graphene supported $\mathrm{Fe}_{3} \mathrm{O}_{4} \mathrm{NPs}$ material between $6-10 \mathrm{mg}$.

Electrochemical Cell Set-up

A LIB using the electrode described above as working electrode and a Li counter electrode was set up. The Li battery cells were assembled into a small glass tube with lithium metal as the counter electrode. A Celgard2325 microporous polypropylene membrane was used as separator, with LBC3015B (Shenyang Kejing Auto-instrument Co.) as electrolyte. The assembling process was carried out inside an argon-filled glove box.

For the SC, the same working electrode was used but with a graphite counter and separator. A $1 \mathrm{M}$ tetraethylammonium tetrafluoroborate $\left(\mathrm{TEABF}_{4}\right.$, Sigma Aldrich Catalog \#242144) in acetonitrile solution was used as the electrolyte with a glass fiber paper as a separator (Whatman® glass microfiber filters, Catalog\# WHA1820055).

\subsubsection{Material Characterization}

The samples were characterized using similar instruments used in the previous project, detailed in Section 2.3.3. In short, a FEI Tecnai F20 TEM equipped with STEM and EDS capabilities, and a Zeiss Sigma VP FEG SEM were used for the analysis of the materials. A Rigaku Ultima IV X-ray Diffraction System (XRD) was used to identify crystallinity of 
the samples. Horiba HR800 UV Raman/Photoluminescence Microprobe Spectrometer was used for surface analysis.

\subsubsection{Electrochemical and Magnetic Measurements}

Electrochemical tests were carried out using a potentiostat (Gamry Instruments, Reference 600 Potentiostat/Galvanostat/ZRA) and a workstation (RST520). The magnetic measurements for LIB were performed in a Quantum Design superconducting quantum interference device (SQUID) magnetometer. The SQUID sample cell was modified to allow in-situ electrochemical and magnetic testing. In-situ measurements were also performed using a VSM at the Applied Magnetics Laboratory, Oregon State University. The ex-situ magnetic measurement was carried out at room temperature using an alternating grating gradient magnetometer (AGM).

\subsection{Results and Discussion}

After rigorous testing and troubleshooting of as-made material and device troubleshooting, the results presented here are those most relevant to the topics discussed thus far.

\subsubsection{Crystalline Structure of $\mathrm{Fe}_{3} \mathrm{O}_{4}$ NPs}

As-made Graphene-Supported $\mathrm{Fe}_{3} \mathrm{O}_{4} \mathrm{NPs}$

While several methods use two precursors as sources for $\mathrm{Fe}^{3+}$ and $\mathrm{Fe}^{2+}$ ions [77], [78], it is possible to use just one precursor in solvothermal synthesis [73], [79]. Here, a simple, green synthesis process is used to produce $\mathrm{Fe}_{3} \mathrm{O}_{4}$ NPs on carbon supports. Building on the synthesis discussed in the previous project, no surfactants or stabilizers are used. While the 
sonochemical process can be used to produce $\mathrm{Fe}_{3} \mathrm{O}_{4} \mathrm{NPs}$, this process requires a complicated set up with precise control over parameters [80]. This is because, unlike Pd and $\mathrm{Au}, \mathrm{Fe}$ is stable in many oxidative states at room temperature. However, it is possible to control the crystal phase of iron oxide using solvothermal process. Hence, here, instead of sonication, the precursors are dissolved in the solvent and the magnetically stirred with the carbon support for $30 \mathrm{~min}$, to achieve uniform dispersion. After this, the solution undergoes the solvothermal process to ultimately form $\mathrm{Fe}_{3} \mathrm{O}_{4}$.

$\mathrm{Fe}_{3} \mathrm{O}_{4}$ NPs hybridized on Graphene
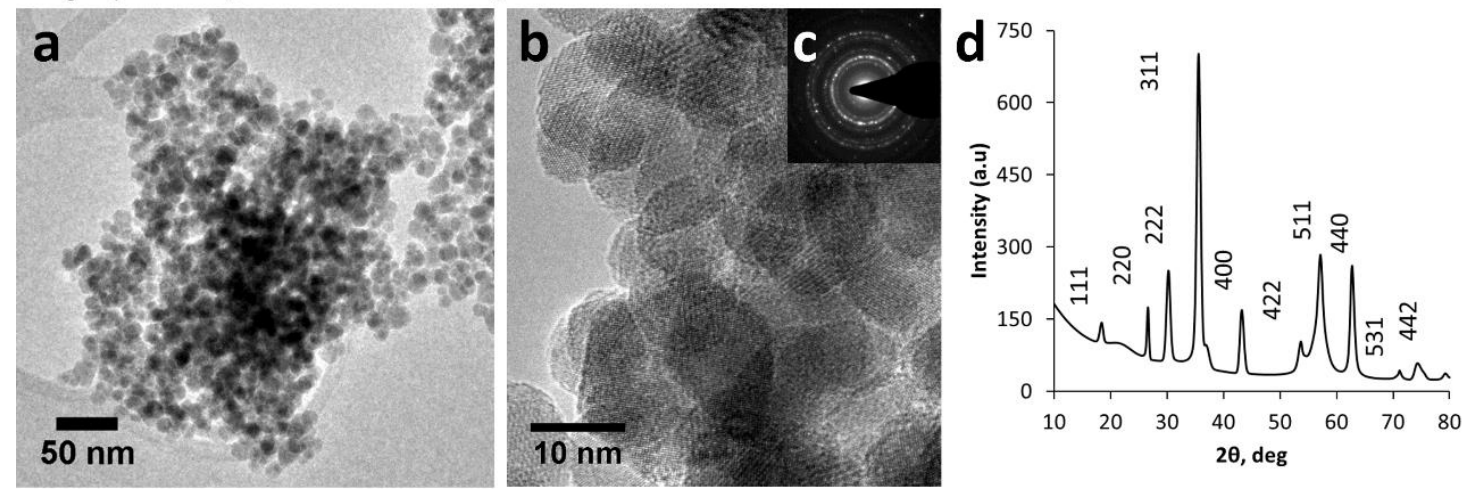

Figure 27. TEM images of $\mathrm{Fe}_{3} \mathrm{O}_{4}$ NPs on graphene at (a) low magnification, (b) high magnification showing lattice fringes and (c) showing an electron diffraction pattern. (d) XRD pattern of these NPs.

Figure 27 is a TEM analysis of this material after it has reacted for 24 hours. Figure 27 (a) shows a low magnification image of the NPs clustered over the graphene, showing uniformly dispersed NPs over the surface of graphene. Figure 27 (b) is taken at higher magnification, showing the NPs are of around $10 \mathrm{~nm}$ in size, with inset (c) showing the electron diffraction pattern generated by these particles, indicating they are highly crystalline. The lattice lines in Figure 27 (b) also show the crystallinity of these NP. The XRD spectrum in Figure 27 (d) confirms the crystal phase of these NPs is spinel $\mathrm{Fe}_{3} \mathrm{O}_{4}$. 
Commercially Produced $\mathrm{Fe}_{3} \mathrm{O}_{4} \mathrm{NPS}$

The as-made graphene-supported $\mathrm{Fe}_{3} \mathrm{O}_{4} \mathrm{NPs}$ specimen was compared against a commercially produced $\mathrm{Fe}_{3} \mathrm{O}_{4}$ NPs, characterized in Figure 28. Despite the NPs being marketed as $20 \mathrm{~nm}$ in size, it is clear the average size of these NPs is much larger with more variations. In Figure 28 (a), at low magnifications, large samples can be seen to cluster together. In Figure 28 (b), some of these NPs are observed at higher magnifications, showing size variations between $100-400 \mathrm{~nm}$. In Figure 28 (c), an edge of one such NPs is observed, revealing a lattice pattern. The XRD spectrum in Figure 28 (d) confirms these NPs are also in the spinel phase of $\mathrm{Fe}_{3} \mathrm{O}_{4}$.

Commercial $\mathrm{Fe}_{3} \mathrm{O}_{4} \mathrm{NPs}$
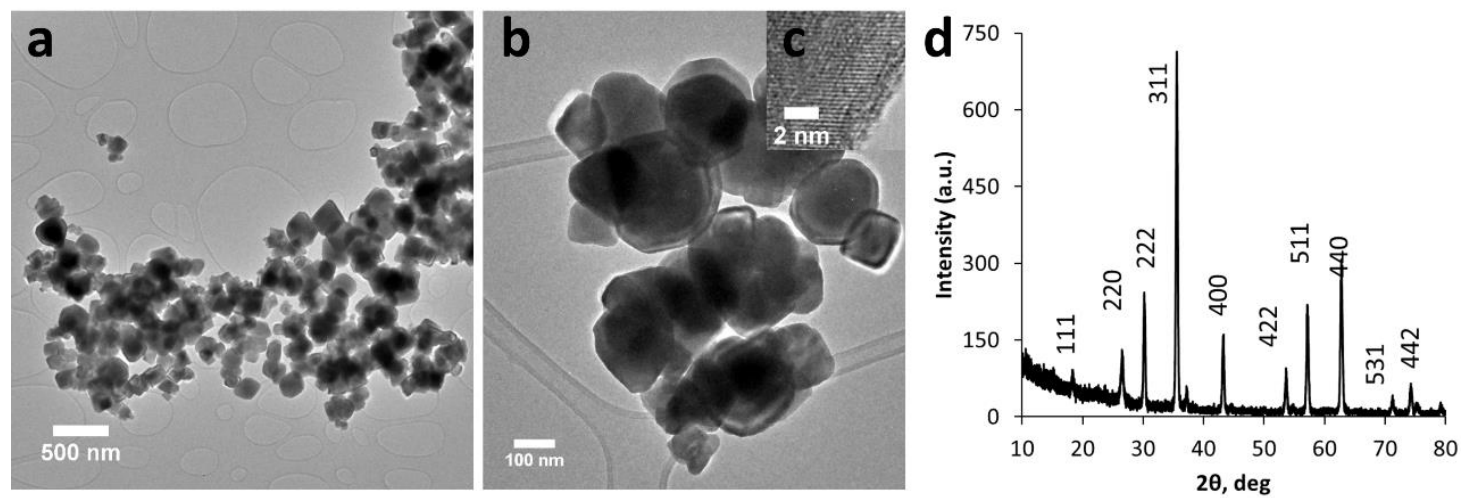

Figure 28. TEM images of (a) low magnification of a cluster of commercial $\mathrm{Fe}_{3} \mathrm{O}_{4} \mathrm{NPs}$. (b) Higher magnification of these NPs, indicating a large variation in size. Inset (c) is an image from an edge, showing lattice fringes. (d) XRD spectrum of NPs, confirming spinel $\mathrm{Fe}_{3} \mathrm{O}_{4}$ NPs phase.

Thus, it is clear that a more precise control over NP size and shape can be achieved using the solvothermal process. This is accuracy is highly desirable, especially in the application of control of magnetic moment. 


\subsubsection{Design of Electrodes to Minimize Binder}

An important aspect of this project is the fabrication of a versatile electrode that can be used for both LIB and SC to facilitate measuring reversible control of magnetic moment. Two primary designs of electrodes were tested for their influence in control of magnetism in electric fields. These designs have been explained in Figure 29. As seen in Figure 29 (a) and (b), first, the conventional design of the electrodes was tested, using both carbon black and graphene respectively. Next, seen in Figure 29 (c), electrodes made using $\mathrm{Fe}_{3} \mathrm{O}_{4}$ NPs directly hybridized on graphene were tested, and the results have been discussed in the next section. Thus, both the influence of carbon black vs graphene and effect of PVDF were analyzed.

a

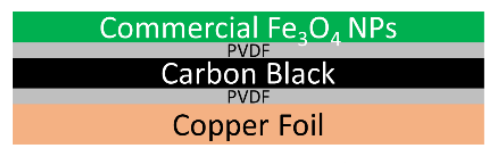

b
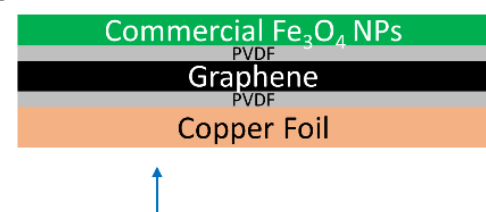

Carbon vs Graphene

C

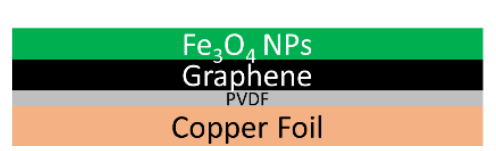

Effect of PVDF

Figure 29. Schematic representation of the electrode designs tested in this project. (a) An electrode made the conventional way, using commercial NPs and carbon black, (b) electrode using commercial NPs but with graphene and (c) the new design of the electrode with NPs directly hybridized onto graphene.

The versatile electrodes were fabricated in way such that they could be used for either LIB or SC testing. All electrodes were characterized before testing, including SEM of the surface before testing. The as-made electrodes of graphene supported $\mathrm{Fe}_{3} \mathrm{O}_{4} \mathrm{NPs}$ and PVDF was characterized as seen in Figure 30. Seen in Figure 30 (a), a low magnification 
SEM image of the surface can be seen, indicating a moderately uniform surface of the dried graphene supported $\mathrm{Fe}_{3} \mathrm{O}_{4}$ NPs and PVDF. In Figure 30 (b), a higher magnification of the surface can be seen, showing some topography, indicating an increased surface and contact area for the electrode-electrolyte interface and finally, Figure 30 (c) is a closer look at the surface of the electrode, focusing on the pores created during drying of the electrodes. Since the electrodes are dried under vacuum, the fluids in the slurry escape by leaving behind these pore structures. This pattern is highly desirable as it allows for increased surface area and wettability of the electrolyte with the surface of the electrodes. The images in Figure 30 indicate the slurry is an even mixture of NPs, carbon support and PVDF.
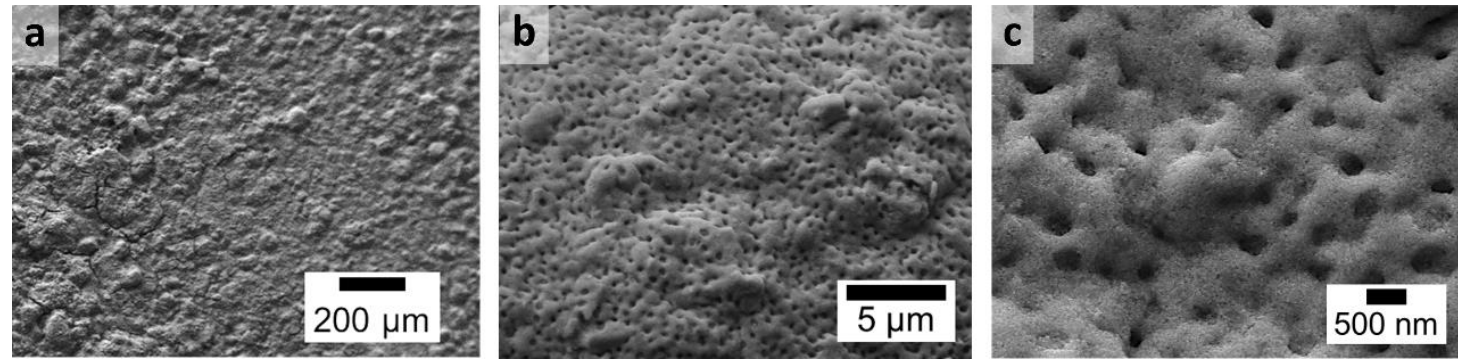

Figure 30. SEM images of the surface of the electrodes at (a) low magnification revealing relatively even surface, (b) higher magnification showing high surface area and (c) highest magnification showing pores where the liquid escaped from the slurry during vacuum drying of the sample.

The other designs of electrodes: commercial $\mathrm{Fe}_{3} \mathrm{O}_{4} \mathrm{NPs}$ with carbon black and PVDF, and commercial $\mathrm{Fe}_{3} \mathrm{O}_{4} \mathrm{NPs}$ with graphene and PVDF yielded similar images during characterization. Hence, these are not included here. This similarity is expected as the design of the electrodes would cause a difference in the appearance of the surface of the electrodes. 


\subsubsection{Control of Magnetic Moment in Electric Fields Generated by LIBs}

In this section, the graphene-supported $\mathrm{Fe}_{3} \mathrm{O}_{4} \mathrm{NPs}$ electrode was tested for change in magnetic moment as a function of electric fields generated by a LIB. Initially, the electrode was characterized in the LIB to ensure the battery was operating as expected. LIBs and other electrochemical cells are usually characterized by two signature tests: cyclic voltammetry (CV) and charge/discharge. For a CV test, the electrode potential is ramped linearly versus time in cyclical phases during which the current is measured. In a typical CV plot, the change in current is plotted as a function of the voltage. The CV is used more as a diagnostic tool to ensure the electrochemical tests are behaving as expected. In a charge/discharge test, constant current is applied until a set voltage is reached. This increase in voltage is usually plotted over time for a charge/discharge plot.

\section{Electrochemical Performance of Graphene-Supported $\mathrm{Fe}_{3} \mathrm{O}_{4} \mathrm{NPS}$ in a LIB}

The CV and the charge/discharge test for the LIB are seen in Figure 31. The CV of the LIB with $\mathrm{Fe}_{3} \mathrm{O}_{4} \mathrm{NP}$ hybridized on graphene electrode is seen in Figure 31 (a). In Figure 31 (b), the results from charge/discharge test are shown, showing good stability and cyclability. The electrodes were found to be stable in the range of $1.5-3 \mathrm{~V}$, which has been used for most LIB tests. 

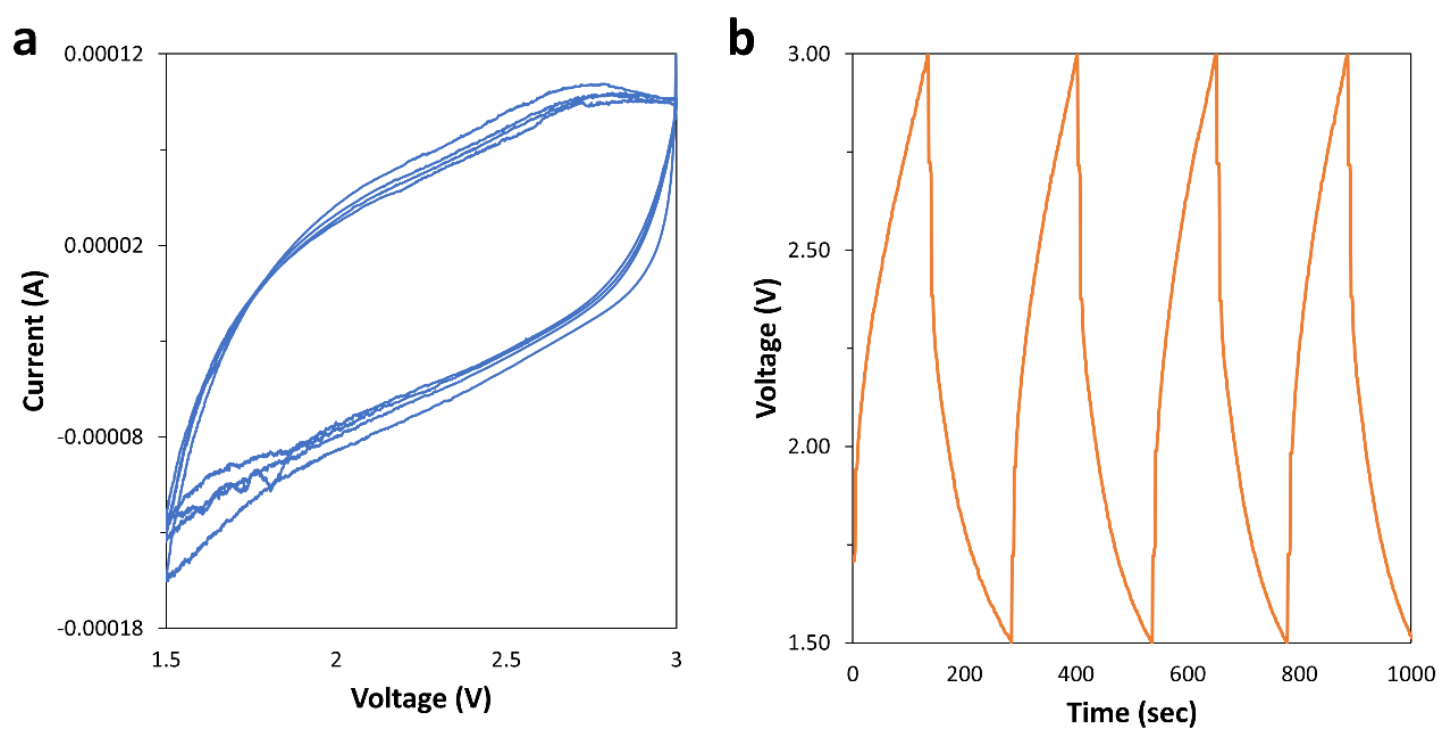

Figure 31. Characteristic curves for LIB with $\mathrm{Fe}_{3} \mathrm{O}_{4} \mathrm{NP}$ hybridized on graphene electrode. (a) CV and (b) charge/discharge curves.

In-Situ Reversible Control of Magnetic Properties of $\mathrm{Fe}_{3} \mathrm{O}_{4} \mathrm{NPs}$ in a LIB

The magnetic testing for LIB with commercial $\mathrm{Fe}_{3} \mathrm{O}_{4}$ NPs, graphene and PVDF were carried out in a SQUID, as seen in Figure 32. The LIBs were made to undergo charge/discharge tests, varying the potential within the fixed range, which varied the electric field generated by the LIB. During this process, the magnetic moment of the $\mathrm{Fe}_{3} \mathrm{O}_{4}$ NPs was recorded, and was found to follow the change in potential correspondingly. This change in magnetic moment was observed to follow the voltage over at least four cycles. In Figure 32 (a), the results of the electrode with commercial NPs bound to graphene was can be seen. The $\mathrm{Fe}_{3} \mathrm{O}_{4}$ NPs in this specimen show a reversible change in magnetization of nearly $2 \%$. That is, simply by using graphene instead of carbon black, a larger change magnetic moment of the material has been achieved increasing from $1 \%$ to $2 \%$. Thus, using 
the more conductive graphene over carbon black is more effective to tune the magnetic properties of materials.

a

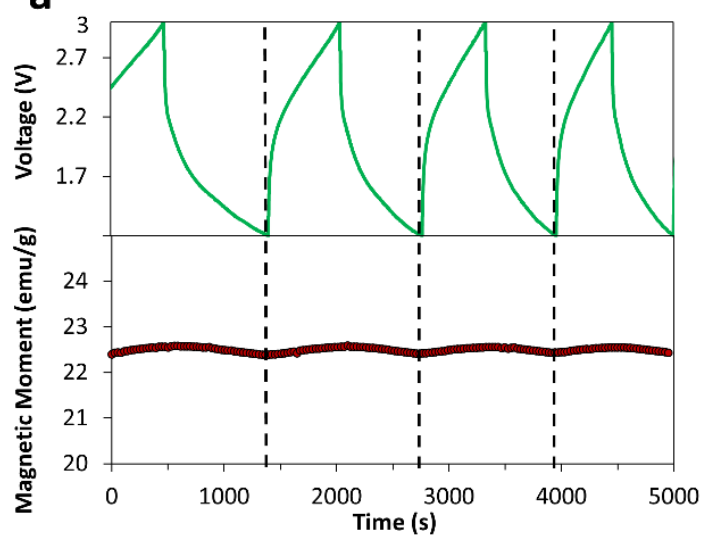

b

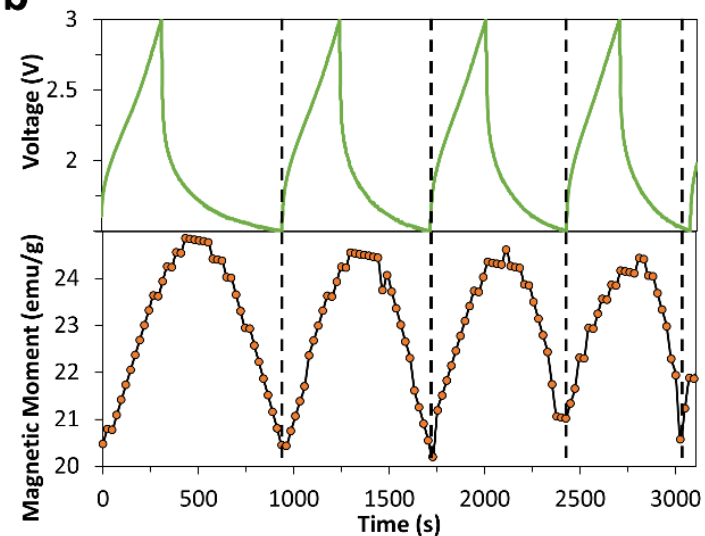

Figure 32. The in-situ magnetic measurements collected during electrochemical discharging/charging of LIB with (a) commercial $\mathrm{Fe}_{3} \mathrm{O}_{4} \mathrm{NPs}$, graphene and PVDF, and (b) graphene supported $\mathrm{Fe}_{3} \mathrm{O}_{4} \mathrm{NPs}$ and PVDF. The saturation magnetization of $\mathrm{Fe}_{3} \mathrm{O}_{4}$ NPs reversibly changes up to $2 \%$ and $18 \%$ respectively for each specimen, as a function of voltage.

Change in Magnetic Properties of $\mathrm{Fe}_{3} \mathrm{O}_{4} \mathrm{NPs}$ in a LIB Measured Ex-Situ

Next, the LIB with $\mathrm{Fe}_{3} \mathrm{O}_{4} \mathrm{NP}$ hybridized on graphene electrode was tested for reversible control of magnetic moment, and the results are shown in Figure 32 (b). In this specimen, the saturation magnetization of $\mathrm{Fe}_{3} \mathrm{O}_{4} \mathrm{NPs}$ reversibly changes from $20.5 \mathrm{emu} / \mathrm{g}$ to $25 \mathrm{emu} / \mathrm{g}$ and back in roughly $17 \mathrm{~min}$. This indicates an $18 \%$ change in magnetic moment. Thus, tuning of magnetic moment has increased greatly when the $\mathrm{Fe}_{3} \mathrm{O}_{4} \mathrm{NPs}$ were directly hybridized on the graphene support. The increased conduction between the NPs and the support has led to an increase in change of magnetic moment.

It is important to note that these tests are occurring at room temperature, and not lower temperatures which were previously necessary for reversible control of magnetism [11]. 
That is, as the LIB cycles through charge/discharge at room temperature, it experiences a reversible change in magnetic moment as a function of the voltage.
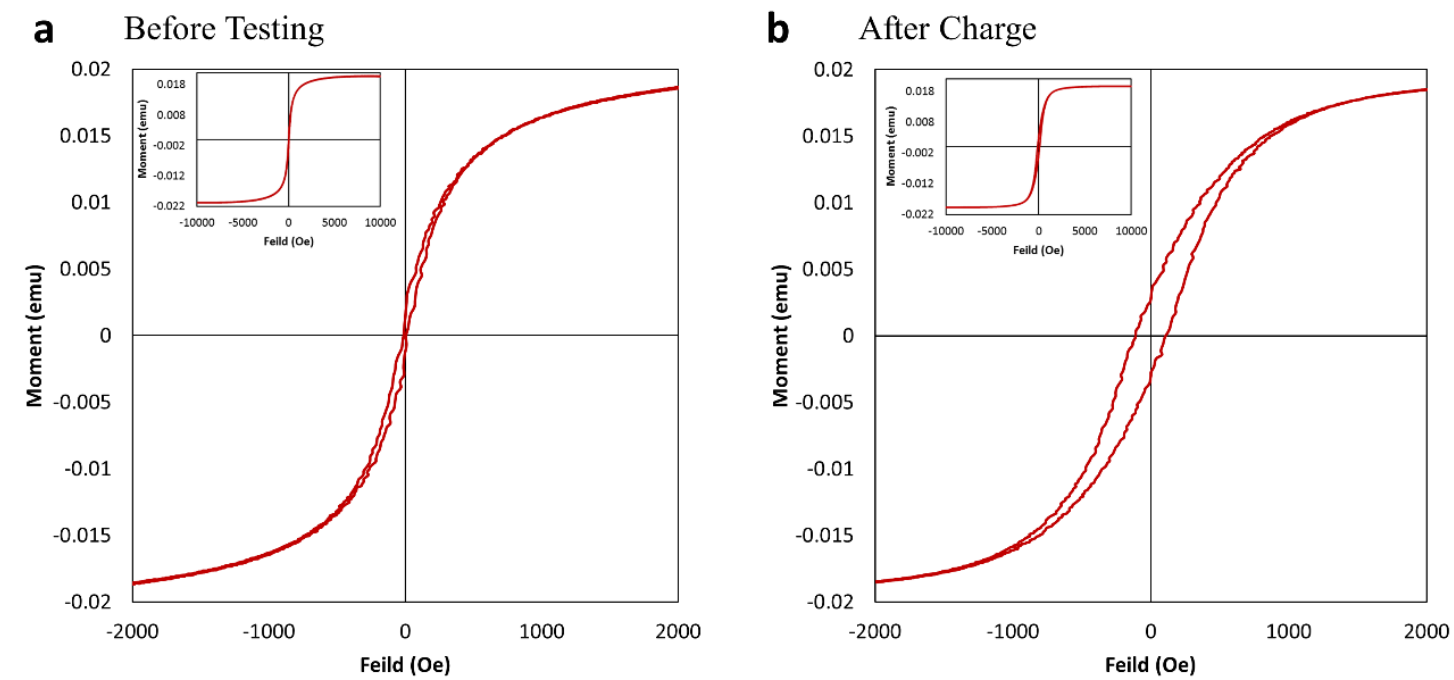

Figure 33. Ex-situ measurement of magnetic moment of the LIB with commercial $\mathrm{Fe}_{3} \mathrm{O}_{4}$ NPs, graphene and PVDF in the (a) original state with inset showing complete data and (b) after charge, with the inset showing total cycle data. The increase in thickness of hysteresis curve indicates a change in magnetic moment.

To further verify that a change in magnetic moment has occurred at room temperature and is retained for at least a short period of time after the test, the magnetic moment of the electrode in the LIB were measured ex-situ in an AGM before and after charge/discharge, seen in Figure 33. Figure 33 (a) is the magnetic moment measurement in an AGM of the commercial $\mathrm{Fe}_{3} \mathrm{O}_{4} \mathrm{NPs}$, graphene and PVDF electrode as-fabricated. A thickness in the hysteresis loop is apparent, characteristic of the sample, with the inset showing the complete data. In Figure 33 (b), the magnetic moment was measured again, this time after the electrode was charged to $3 \mathrm{~V}$. The thickness of the hysteresis has increased, indicating a definite change in magnetic moment. The inset once again shows the complete data. 


\subsubsection{Mechanism of Magnetic Switching of Graphene-Supported $\mathrm{Fe}_{3} \mathrm{O}_{4} \mathrm{NPs}_{\text {in }} \mathrm{LIBs}$ Role of ME Coupling of Multiferroic Materials}

The ability to influence in magnetic properties by exposure to electric fields is due to an intrinsic property of the material, called the ME effect. The ME effect is the coupling between the magnetic and the electric properties of a material. That is, change in one property effects another property, for example, a change to the electrical properties of the material would also affect the magnetic properties of the material. This phenomenon is often observed in multiphase multiferroic compounds, such as specific transition metals compounds [11]. While there are several approaches in trying to switch the magnetic moments of a material [11], here the aim is to manipulate the net ferrimagnetism exhibited by spinel crystal structures.

\section{Effect of ME Coupling in Spinel Crystal Structures}

This approach has been used on spinel crystal structures like $\gamma-\mathrm{Fe}_{2} \mathrm{O}_{3}$ [7], [8], $\mathrm{MnFe}_{2} \mathrm{O}_{4}$ [8], $\mathrm{ZnFe}_{2} \mathrm{O}_{4}, \mathrm{CuFe}_{2} \mathrm{O}_{4}$ [9] and even $\mathrm{Fe}_{3} \mathrm{O}_{4}$ in the past [10]. The ability to change the magnetic moment in spinels lies in the lattice positions of the cation and anions in the crystal structures. The spinel crystal structure and its net magnetic moment has been represented in Figure 34. Seen in Figure 34 (a), the spinel structure is a cubic crystal system, with the oxide anions arranged in a cubic close-packed lattice and the two cations $\mathrm{A}^{+2}$ and $\mathrm{B}^{+3}$ (in this case, $\mathrm{Fe}^{+2}$ and $\mathrm{Fe}^{+3}$ ) occupying some or all of the octahedral and tetrahedral sites in the lattice. $\mathrm{Fe}_{3} \mathrm{O}_{4}$ has an inverse spinel structure, which means half of the $\mathrm{Fe}^{3+}$ cations occupy tetrahedral sites while the other half, along with $\mathrm{Fe}^{2+}$ cations, occupy octahedral sites. Seen in Figure 34 (b), the ions occupying the octahedral sites tend 
to have magnetic moments opposing the magnetic moment exhibited by the ions in the tetrahedral sites. This causes an overall net magnetic moment for the spinel structure.
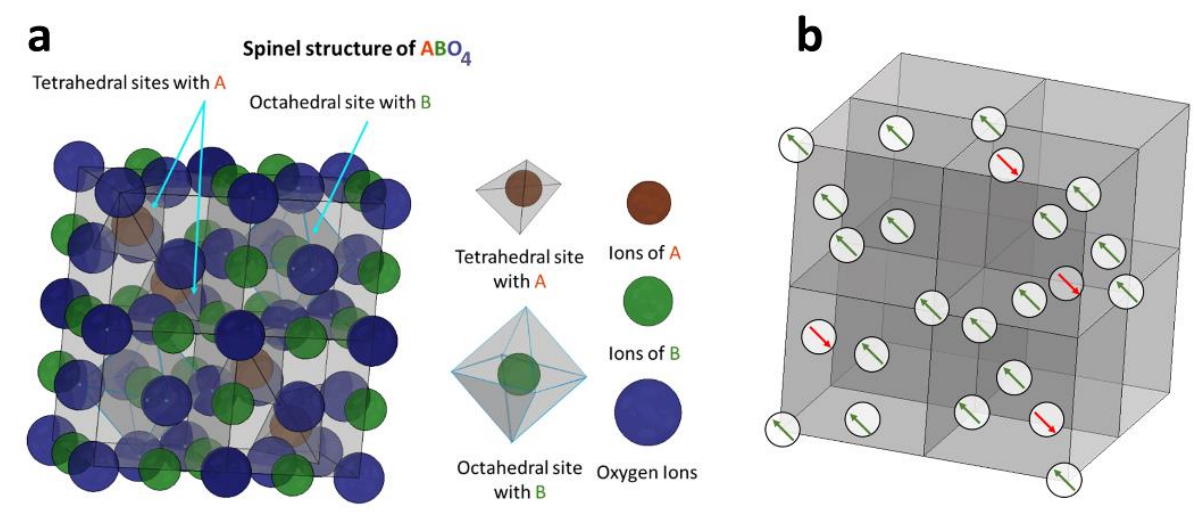

Net magnetic moment

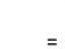

Figure 34. 3D representation of the crystal structure of $\mathrm{Fe}_{3} \mathrm{O}_{4}$ (a) showing locations of cation $\mathrm{A}\left(\mathrm{Fe}^{2+}\right.$ ions) in octahedral sites and cation $\mathrm{B}\left(\mathrm{Fe}^{3+}\right.$ ions) split across the remaining octahedral and tetrahedral sites; (b) showing the magnetic moments of the ions, and the net magnetic moment.

\section{Influence of Superexchange Interaction in Spinel Crystal Structures}

Aside from inherent magnetic moments generated by the internal spin of electrons, the net magnetic moment of the ions is also controlled in part by the superexchange interaction with the neighboring ions. The phenomenon of superexchange interactions has been illustrated in Figure 35. Superexchange interaction is a strong coupling between two nextto-nearest neighbor cations through a non-magnetic anion. It is a result of electrons from the same donor atom being coupled with the receiving ions' spins. If the two next-to-nearest neighbor positive ions are connected at $90^{\circ}$ and higher to the bridging non-magnetic anion, as seen in Figure 35 (a), ferromagnetism can be expected. If this angle between the ions is $120^{\circ}$ and higher, as seen in Figure 35 (b), then the interaction leads to antiferromagnetism. Thus the superexchange interactions between the $\mathrm{Fe}^{2+}, \mathrm{Fe}^{3+}$ cations and $\mathrm{O}^{2-}$ anion leads to a net ferrimagnetic property [81]. This ability to affect change to the magnetic properties 
of the material makes spinels crystal structure a popular choice for applications of control of magnetism. [7]-[10].
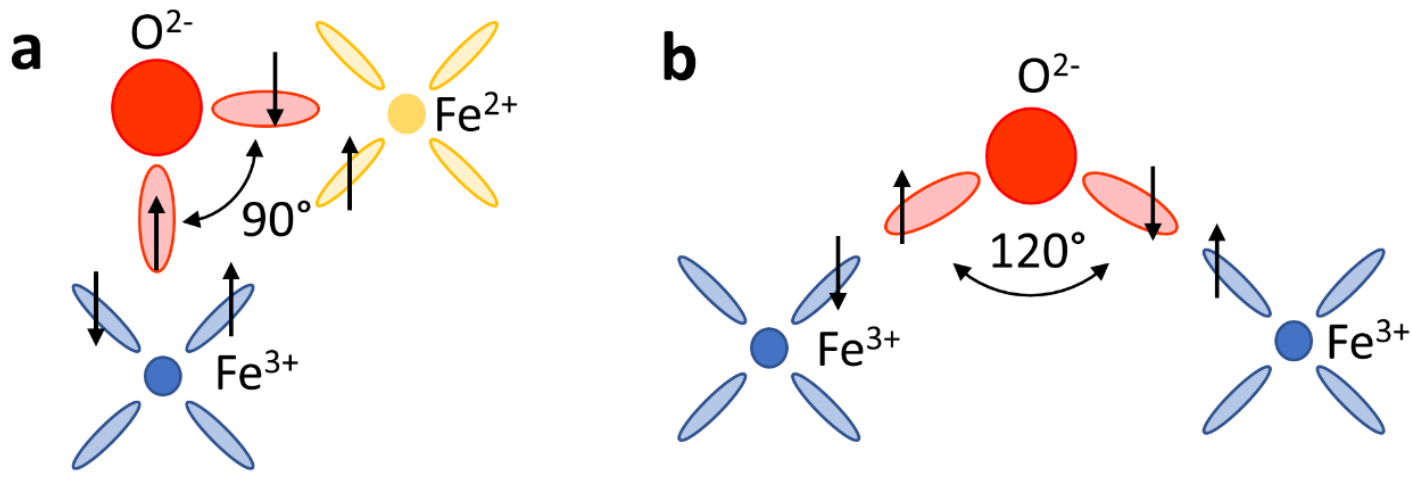

Figure 35. Superexchange interactions between the $\mathrm{Fe}^{3+}$ and $\mathrm{Fe}^{2+}$ cations and the $\mathrm{O}^{2-}$ anion, (a) when the angle between the ions is $90^{\circ}$ usually leading to ferromagnetism and (b) when the angle between the ions is $120^{\circ}$ usually leading to antiferromagnetism.

\section{Mechanism of Change in Magnetic Moment of $\mathrm{Fe}_{3} \mathrm{O}_{4}$ in a $\mathrm{LIB}$}

Based on the above discussions on the structure of spinels and magnetic properties, the following mechanism is considered to take place in a LIB that allows for a change in magnetism of a material. This mechanism involves intercalation, which refers to the reversible insertion of $\mathrm{Li}$ ions into the working electrode when the cell is charged [82]. During the discharge of LIB, the highly potent and mobile $\mathrm{Li}^{+}$ions intercalate in several locations in the lattice of the spinel structure, including in interstitial locations [83]. Consequently, the $\mathrm{Li}^{+}$ions and neighboring ions undergo new superexchange interactions, which result in a variation in magnetic moment. As the voltage increases, the influx of $\mathrm{Li}^{+}$ ions intercalating with the spinel structure increases, continuing the change the magnetic moment. Upon charging, the $\mathrm{Li}^{+}$ions deintercalate, leaving behind the original spinel structure and reverting the magnetic moment to its original value. Thus, in this system, the magnetic moment change is a fundamental property the material structure. 


\section{Drawbacks of Li intercalation}

While LIBs have been successful at manipulating the magnetic moment of materials in the past, recently, efforts are being made to move away from using LIBs to drive the magnetic moment of materials [70], [84]. This is because of some inherent drawbacks of the very same potent and mobile $\mathrm{Li}^{+}$ions. During charge, there is a chance that the $\mathrm{Li}^{+}$ions do not fully deintercalate, meaning some of the $\mathrm{Li}^{+}$ions would be remaining in the crystal structure of $\mathrm{Fe}_{3} \mathrm{O}_{4}$. These extra ions cause deformities in the matrix, producing strain and sometimes leading to material cracking and failure [85]. This generally happens if the LIB is not operated under the right voltage range.

One example of this is the CV seen in Figure 36. The original locations of the redox peaks in Cycle 1, at $0.75 \mathrm{~V}$ and $2 \mathrm{~V}$ respectively, disappear over consecutive cycles. 36 This is due to the intercalation of $\mathrm{Li}^{+}$ions, which cause an irreversible change over every cycle. In other words, over time, the $\mathrm{Li}^{+}$ions build up in the spinel structure. This causes a

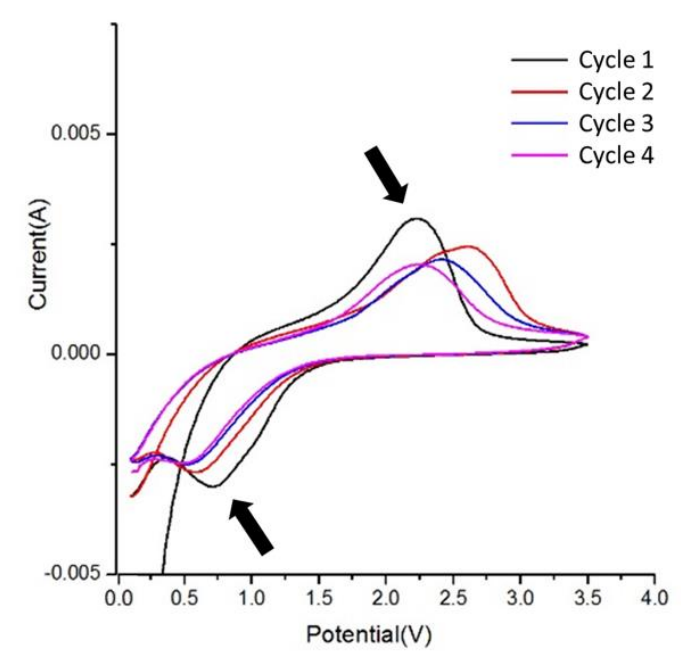

Figure 36. The CV of a LIB showing damage to material, indicated by the disappearing peaks. Arrows point to peaks of first cycle. lot of strain on the crystal matrix, which eventually changes to rock salt crystal structure to accommodate the additional ions. The rock salt structure is non-magnetic, making unfavorable for this project. Hence, it is important to test the LIB in the set voltage that prevents conversion from spinel $\left(\mathrm{Fe}_{3} \mathrm{O}_{4}\right)$ to rock salt structure $\left(\mathrm{Li}_{2}\left(\mathrm{Fe}_{3} \mathrm{O}_{4}\right)\right)$, as observed by others in the past also [7], [10]. 
Therefore, while LIBs have shown success in reversible change of magnetic moments, their limitations prevent complete dependability on this device for this application.

\subsubsection{Mechanism of Magnetic Switching of Graphene-Supported $\mathrm{Fe}_{3} \mathrm{O}_{4} \mathrm{NPs}_{\text {in }} \mathrm{SCs}$} Mechanism of Change in Magnetic Moment of $\mathrm{Fe}_{3} \mathrm{O}_{4}$ without $\mathrm{Li}^{+}$ions

Interest has been growing in finding alternatives to overcome the limitations of LIB. Recently, the use of hybrid SCs for generating electric fields to manipulate magnetic moment has seen a $2 \%$ change in magnetic moment. However, this change was observed using $\mathrm{La} 0.74 \mathrm{Sr}_{0.26} \mathrm{MnO}_{3}$ (LSMO) material, which has a perovskite crystal structure [70].

In the absence of $\mathrm{Li}^{+}$ions, it is difficult to achieve the same permeability into the crystal structure of an electrode. However, the phenomenon of pseudocapacitance of a SC has a similar working principle. Pseudocapacitance is the charge transfer caused by a very fast sequence of reversible redox reactions, electrosorption or intercalation processes on the surface of electrodes [86], [87]. This means pseudocapacitance is generally limited to the surface of the electrodes. Nevertheless, since no $\mathrm{Li}^{+}$ions are present in the setup, the system is more flexible for manipulations. This makes hybrid SCs promising devices to manipulate magnetic moments of materials.

Hybrid SCs have been shown to be successful when changing the magnetic properties of perovskites [70]. The perovskite crystal structure is different from the spinel crystal structure, with a cubic structure. The edges are occupied by the $\mathrm{La}^{3+}$ or $\mathrm{Sr}^{2+}$ ions, the face centered locations are occupied by oxides and the body centered location is occupied by $\mathrm{Mn}^{3+}$. However, magnetic moment for these structures is also strongly influenced by the 
superexchange interactions between the ions. For this reason, change in magnetic moment can be expected with materials having spinel crystal structures. In this section, efforts were made to observe change in magnetic moment of $\mathrm{Fe}_{3} \mathrm{O}_{4} \mathrm{NPs}$ in a SC. Thus far, to the best of the author's knowledge, $\mathrm{Fe}_{3} \mathrm{O}_{4}$ NPs have not been tested in a SCs for variations in magnetic moments.

Design of Supercapacitor for Testing Electromagnetic Properties of $\mathrm{Fe}_{3} \mathrm{O}_{4}$
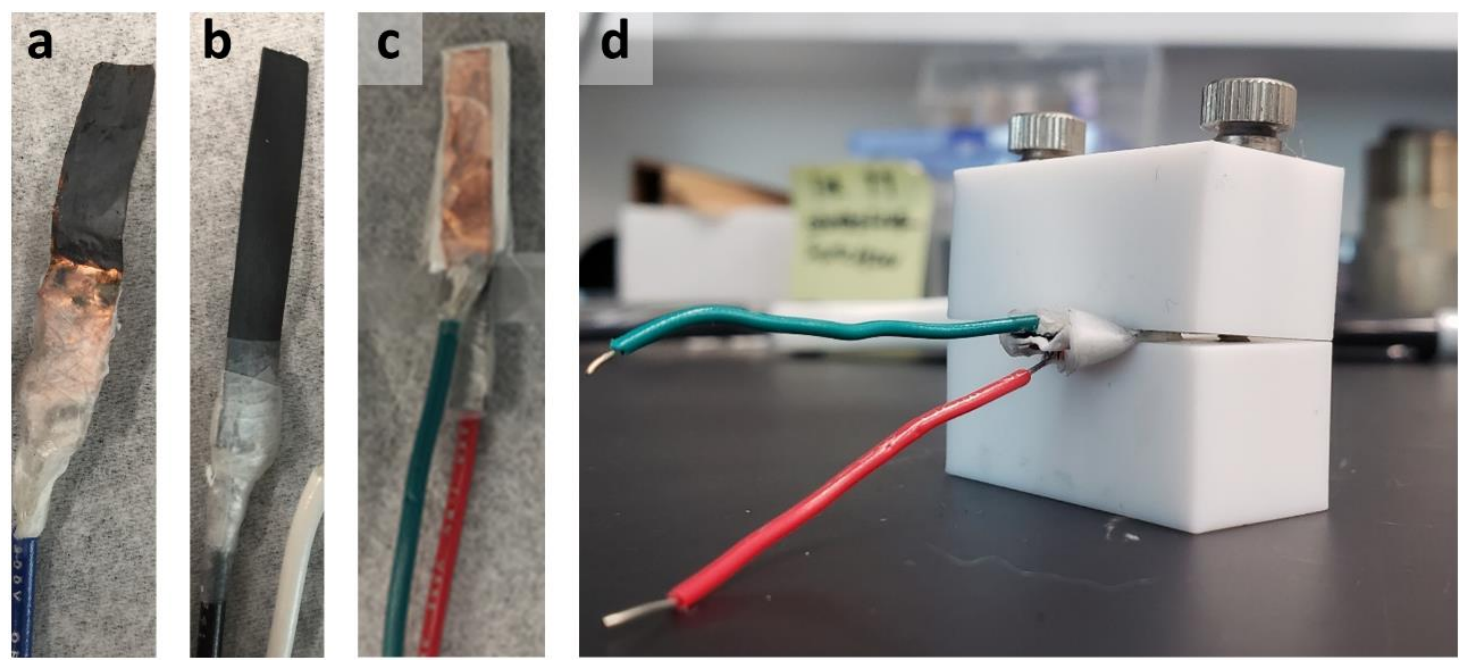

Figure 37. Construction of SC. (a) working electrode with graphene supported $\mathrm{Fe}_{3} \mathrm{O}_{4}$ NPs, (b) carbon counter, (c) the assembled SC and (d) the clamp system used to test the SC.

For the reasons listed above, in this project, a hybrid SC was designed and built to test the electrical and magnetic properties of the active material. For consistency with the LIB testing system, $\mathrm{Cu}$ foil was continued to be used as the current conductor for the working electrode. $\mathrm{Cu}$ foil has some advantages in that it is diamagnetic in nature and thus does not interfere with the magnetic signal of the material. However, $\mathrm{Cu}$ is easily oxidized, and 
hence the rest of the $\mathrm{SC}$ was built around preventing the $\mathrm{Cu}$ from oxidizing while showing high capacitance.

Some images from the construction of the SC can be seen in Figure 37. Pictured in Figure 37 (a) is the graphene supported $\mathrm{Fe}_{3} \mathrm{O}_{4} \mathrm{NPs}$ bound to the $\mathrm{Cu}$ foil with PVDF. In Figure 37 (b), the carbon counter electrode is pictured. These electrodes were assembled in Figure 37 (c) with a glass fiber separator. Finally, to ensure even contact, the SC was tested clamped together with screws, as seen in Figure 37 (d).

Electrochemical Performance of Graphene-Supported $\mathrm{Fe}_{3} \mathrm{O}_{4} \mathrm{NPS}$ in a $\mathrm{SC}$
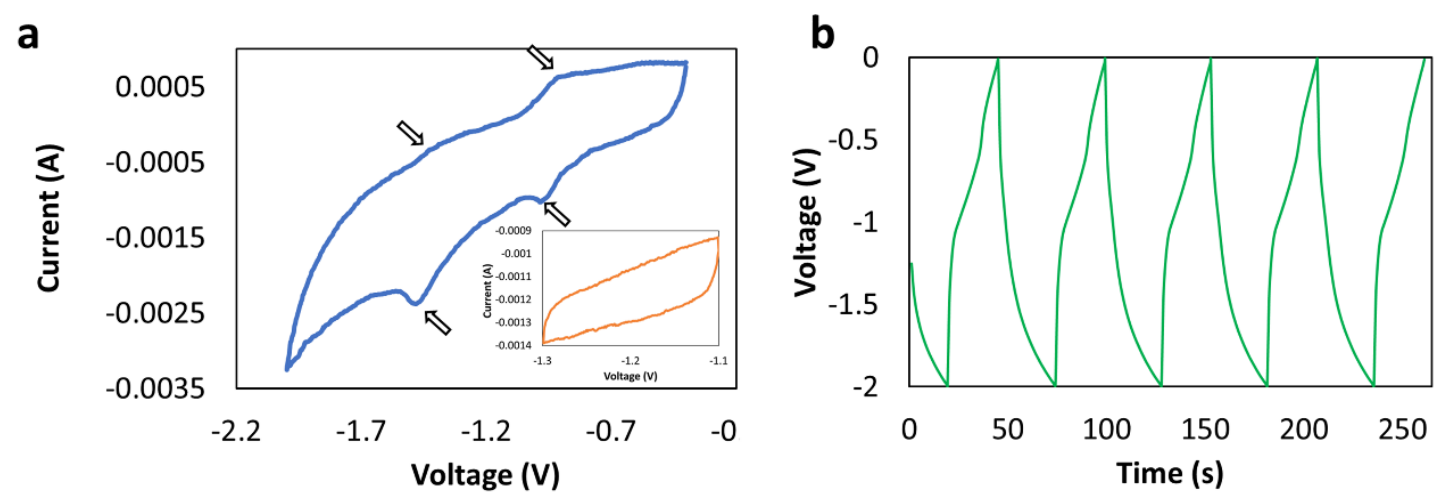

Figure 38. Characteristic curves for $\mathrm{SC}$ with $\mathrm{Fe}_{3} \mathrm{O}_{4} \mathrm{NP}$ hybridized on graphene electrode. (a) CV showing redox peaks, as indicated by the arrows, with inset showing EDLC layer behavior of SC, and (b) charge/discharge curves.

The constructed SCs were characterized using CV and the charge/discharge test. These two characteristic tests for SCs with graphene supported $\mathrm{Fe}_{3} \mathrm{O}_{4}$ NPs as electrodes can be seen in Figure 38.

Seen in Figure 38 (a), the CV reveals the redox peaks of $\mathrm{Fe}^{3+} / \mathrm{Fe}^{2+}$ as indicated by the arrows. These peaks indicate the $\mathrm{SC}$ is exhibiting pseudocapacitance. The inset shows $\mathrm{CV}$ 
for the same SC in lower voltage range with electric double layer capacitor (EDLC) behavior (i.e. no peaks).

The charge/discharge curves for this SC can be seen in Figure 38 (b), showing rapid charge/discharge cycles. The speed of these cycles can be controlled by increasing or decreasing the current. These curves show characteristic behavior of a SC, thus giving confidence to the device built in this project for the testing.

\subsubsection{Control of Magnetic Moment in Electric Fields Generated by SCs}

Change in Magnetic Properties of $\mathrm{Fe}_{3} \mathrm{O}_{4} \mathrm{NPs}$ in a SC Measured Ex-Situ

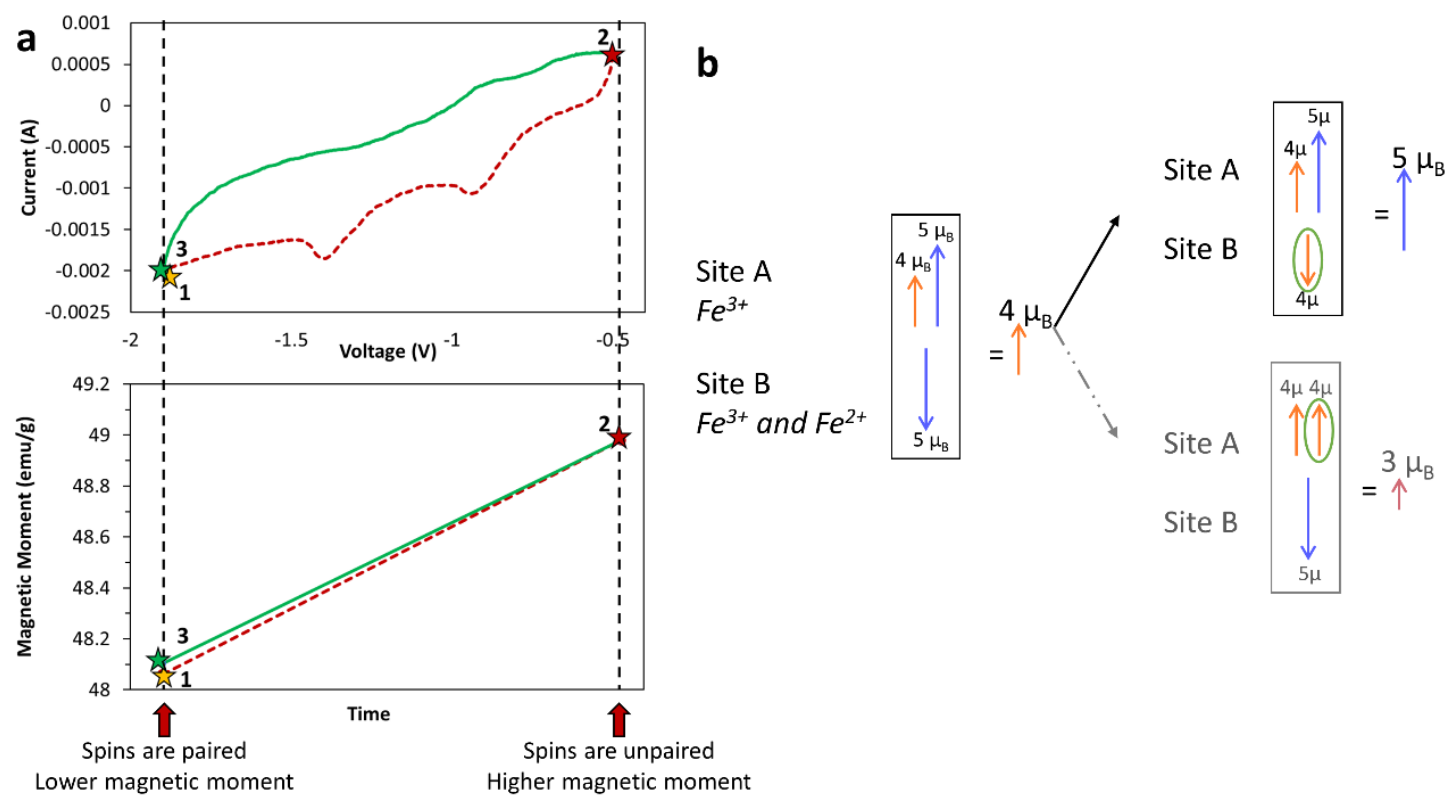

Figure 39. (a)Change in magnetic moment as a function of voltage for $\mathrm{Fe}_{3} \mathrm{O}_{4} \mathrm{NPs}$ hybridized on graphene as tested in a SC.(b) Schematic explanation for the change in magnetic moment for this SC.

Using the $\mathrm{SC}$ described in the previous sections, the graphene supported $\mathrm{Fe}_{3} \mathrm{O}_{4} \mathrm{NPs}$ as electrodes was tested for change in magnetic moment. These results have been recorded in Figure 39. 
Seen in Figure 39 (a), the change in magnetic moment was recorded as a function of $\mathrm{CV}$. The magnetic moment of the electrode was measured at 3 stages. First, the magnetic moment was measure for the electrode as-is ex-situ in the VSM, called point 1. Next, the $\mathrm{CV}$ test was started and stopped after half a cycle (charge). At this stage, the electrode was tested in a VSM for net magnetic moment, called point $\mathbf{2}$. Finally, the cycle was completed (discharge) and the magnetic moment was measured as point 3 . Thus, a change of magnetic moment of $2 \%$ was observed in this test.

Based on the interactions between the surface of the electrode and the electrolyte, the two conditions for the magnetic moment can be expected, as seen in Figure 39 (b). The image to the left in Figure 39 (b) represents the ground state of $\mathrm{Fe}_{3} \mathrm{O}_{4}$ NPs. If the surface and superexchange interactions lead to a greater number of unpaired electrons, the upper situation in Figure 39 (b) can be expected, with an overall increase in net magnetic moment. However, in a situation where the electrons are spin paired, the lower part of Figure 39 (b) can be expected, with a net decrease in magnetic moment.

\subsubsection{Construction of Supercapacitor to Measure Change in Magnetic Moment of} $\mathrm{Fe}_{3} \mathrm{O}_{4} \mathrm{NPs}$

Effect of Configuration, Current-Collector and Electrolyte on Performance of SC

Initially, a three-electrode cell configuration capacitor was initially set up with activated carbon and graphite, using $\mathrm{Ni}$ foam as the metal conductor. Upon further refinement, a three-electrode cell capacitor using graphene supported $\mathrm{Fe}_{3} \mathrm{O}_{4} \mathrm{NPs}$ on Ni foam, a counter electrode of $\mathrm{Ni}$ foam and reference electrode was constructed. However, $\mathrm{Ni}$ is weakly paramagnetic, so $\mathrm{Cu}$ was used as a current collector instead. Additionally, 
while metal foils offer increase in surface area, the slurry containing $\mathrm{Fe}_{3} \mathrm{O}_{4}$ NPs, carbon and PVDF could not be deposited evenly on the porous surface. For these reasons, experiments using $\mathrm{Ni}$ and foam were discontinued.
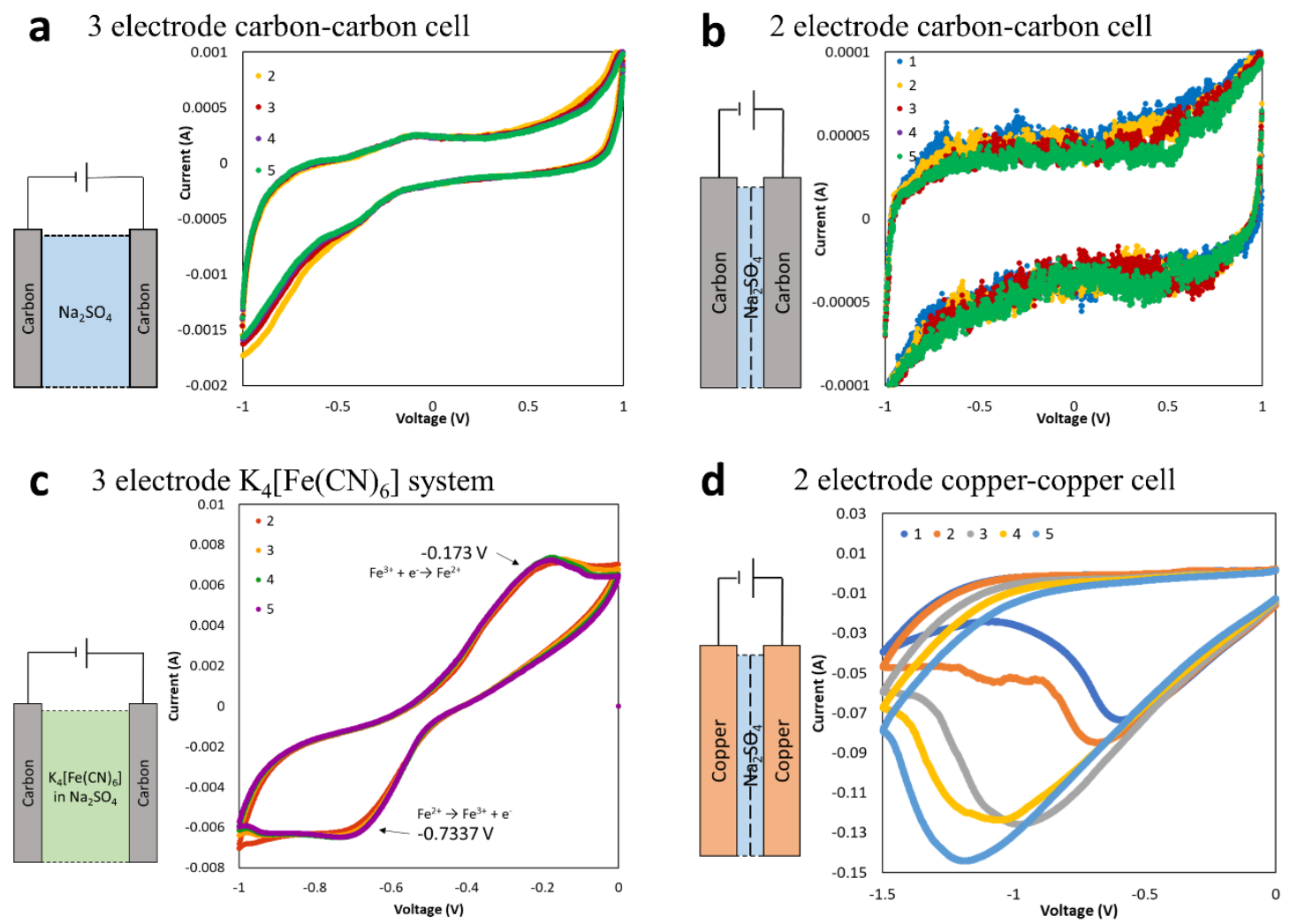

Figure 40. Different configurations of the SC with corresponding CVs. (a) threeelectrode carbon-carbon SC, (b) two-electrode carbon-carbon SC, (c) three-electrode carbon-carbon $\mathrm{SC}$ with potassium ferrocyanide to identify redox peaks of $\mathrm{Fe}^{2+} / \mathrm{Fe}^{3+}$ and (d) two-electrode $\mathrm{Cu}-\mathrm{Cu} \mathrm{SC}$.

Several other parameters were varied for the construction of the SC. Some of these designs are shown in Figure 40. Seen in Figure 40 (a), initially a three-electrode carboncarbon cell was built followed by a two-electrode carbon-carbon SC, seen in Figure 40 (b). The CV curves from this system helped to troubleshoot and for reproducibility. Figure 40 (c) is of a three-electrode carbon-carbon SC with potassium ferrocyanide in the electrolyte 
to identify redox peaks of $\mathrm{Fe}^{2+} / \mathrm{Fe}^{3+}$. These peaks are visible at -0.734 and $-0.173 \mathrm{~V}$ respectively. Finally, seen in Figure 40 (d) a three-electrode symmetric $\mathrm{Cu}-\mathrm{Cu} \mathrm{SC}$ was built and analyzed, the corresponding CV curves show the oxidation of the $\mathrm{Cu}$ foil.

\section{Cyclability of Electrode Material}
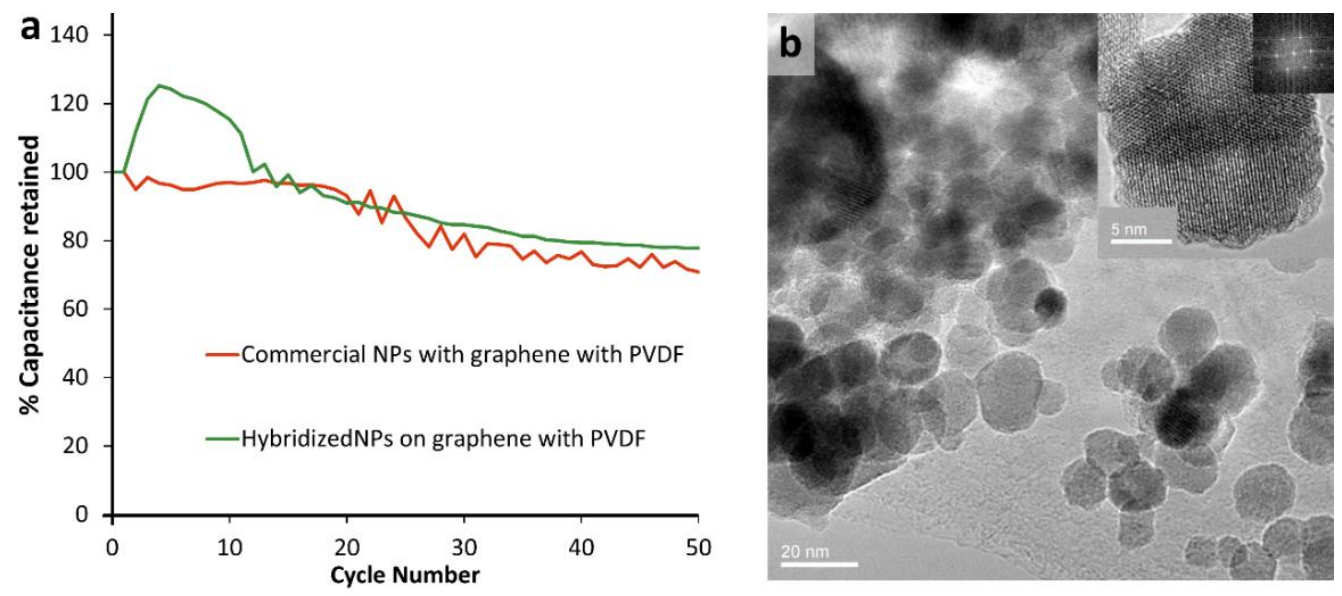

Figure 41. (a) Test of cyclability of the electrodes, plotting capacitance retained over cycle number. (b) TEM images of synthesized $\mathrm{Fe}_{3} \mathrm{O}_{4}$ NPs after 1000 cycles, with inset (c) showing crystallinity is retained by the NPs after this long cycling.

The cyclability of the electrodes was tested, and results are shown in Figure 41. The electrodes were cycled as SC for 50 cycles and the \% capacitance retained was mapped as a function of the cycle number, seen in Figure 41 (a). This test was performed to study the effect of PVDF, hence two corresponding samples were tested against each other: commercial $\mathrm{Fe}_{3} \mathrm{O}_{4}$ NPs with graphene and $\mathrm{Fe}_{3} \mathrm{O}_{4}$ NPs hybridized directly onto the graphene. Initially, in the first 10 cycles, an increase in capacitance is seen with the graphene supported $\mathrm{Fe}_{3} \mathrm{O}_{4} \mathrm{NPs}$, this is because the commercial $\mathrm{Fe}_{3} \mathrm{O}_{4}$ NPs with graphene has additional PVDF that would interfere with the capacitance of the SC. Over time, both 
SCs stabilize, with the graphene supported $\mathrm{Fe}_{3} \mathrm{O}_{4}$ NPs retaining more capacitance over time.

The SC with graphene supported $\mathrm{Fe}_{3} \mathrm{O}_{4}$ NPs electrode was allowed to run for up to 1000 cycles, after which the active material, graphene supported $\mathrm{Fe}_{3} \mathrm{O}_{4} \mathrm{NPs}$, was recovered. This material was analyzed in the TEM, as seen in Figure 41 (b). A large variation in size can be seen, suggesting the NPs underwent some rearrangement and damage in the 1000 cycles. The inset Figure 41 (c) and the FFT show the crystallinity of the recovered NPs, indicating despite damage, some original characteristics remain in the sample.

\subsubsection{Mechanism of Formation of Graphene-Supported $\mathrm{Fe}_{3} \mathrm{O}_{4} \mathrm{NPs}$}

In order to optimize the material, formation of $\mathrm{Fe}_{3} \mathrm{O}_{4} \mathrm{NPs}$ was studied systematically and in detail. Here, some of these results have been presented.

\section{Effect of Synthesis Time on Phase and Crystal Structure of Iron Oxide NPS}

To make $\mathrm{Fe}_{3} \mathrm{O}_{4} \mathrm{NPs}$, iron (III) acetylacetonate, $\mathrm{Fe}\left(\mathrm{C}_{5} \mathrm{H}_{7} \mathrm{O}_{2}\right)_{3}$ precursor was dissolved in ethanol, mixed with graphene and heated to $180^{\circ} \mathrm{C}$. While XRD is useful for identification of crystal structure, such as spinel vs perovskite, it cannot clearly differentiate between phases of a material that have the same crystal structure, such as $\gamma-\mathrm{Fe}_{2} \mathrm{O}_{3}$ and $\mathrm{Fe}_{3} \mathrm{O}_{4}$ since both these phases have inverse spinel crystal structure [7], [10]. For this reason, Raman spectroscopy was used to confirm both the crystal structure and phase of the iron oxide produced using the method described in Section 3.3.1. While the complete mechanism is 
out of the scope of this study, efforts were made to understand the basic formation of the $\mathrm{Fe}_{3} \mathrm{O}_{4}$ NPs.

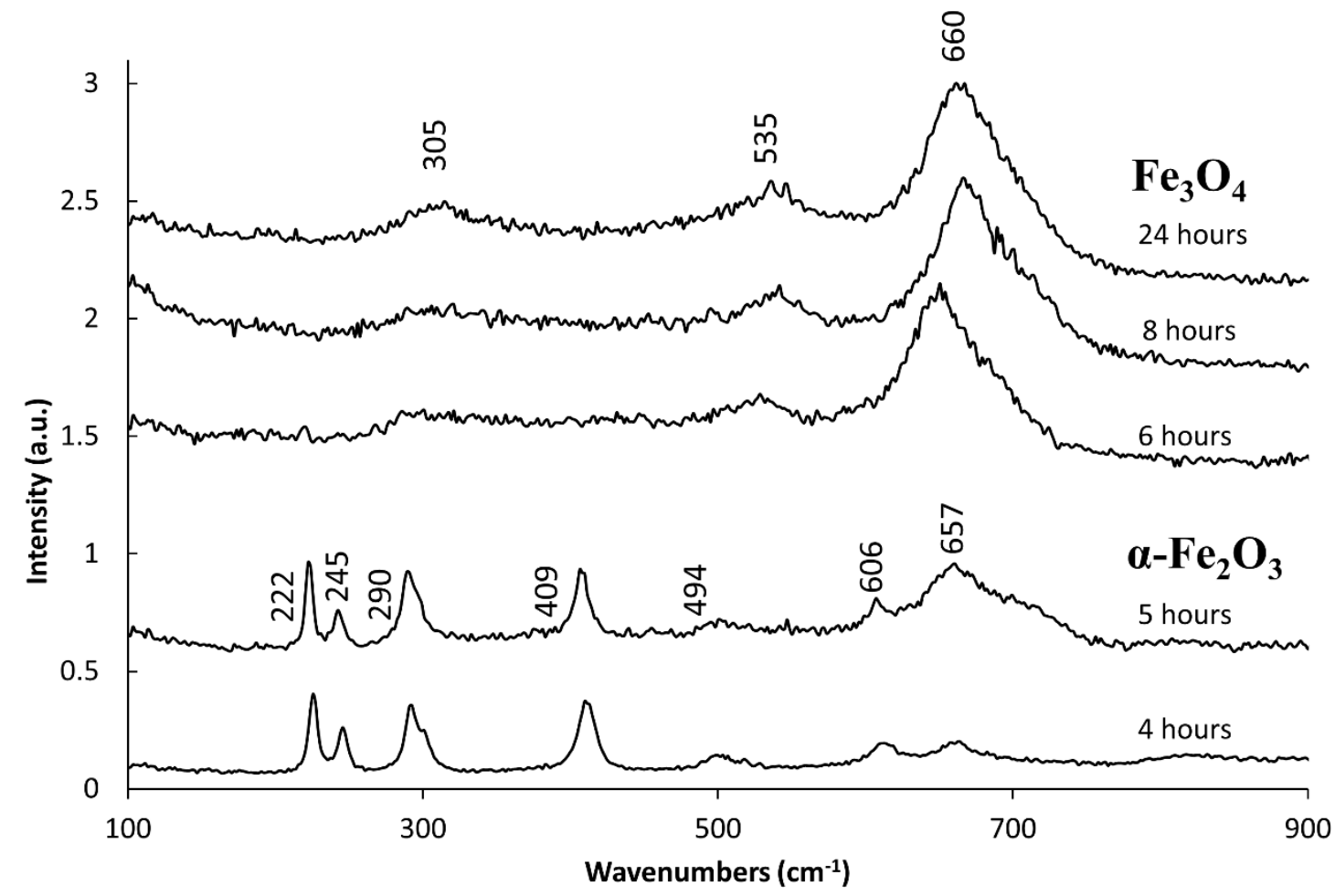

Figure 42. Raman spectra of iron oxide formed via solvothermal synthesis in ethanol at different synthesis times.

With an intent to identify when the $\mathrm{Fe}_{3} \mathrm{O}_{4}$ phase is formed during the synthesis process, the reaction was systematically studied at hourly intervals. The recovered materials were characterized using Raman spectroscopy. Of the various experiments, samples recovered between 4 to 8 hours were found be most interesting and have been shown in Figure 42 along with the final material formed after 24 hours. The spectra of the specimen with 24 hours synthesis matched the spectra of $\mathrm{Fe}_{3} \mathrm{O}_{4}$, confirming the spinel structure is formed using this process [88]. Additionally, the spectra of the 4-hour 
reaction time sample vary greatly from the 24 hours sample. Interestingly, these peaks were found to correlate to $\alpha-\mathrm{Fe}_{2} \mathrm{O}_{3}$ phase [89], [90]. This spectrum suggests $\alpha-\mathrm{Fe}_{2} \mathrm{O}_{3}$ as a possible intermediary phase in the formation of $\mathrm{Fe}_{3} \mathrm{O}_{4}$ phase. After 5 hours, a peak at 660 $\mathrm{cm}^{-1}$ begins to grow prominence in the spectra, indicating a mixed phase. 6 hours into the reaction the NPs appear to have reached $\mathrm{Fe}_{3} \mathrm{O}_{4}$ phase, and spectra are consistent from there on.

Mechanism of Formation of $\mathrm{Fe}_{3} \mathrm{O}_{4}$ Phase via Solvothermal Process

Thus, based on these findings and previous reports [91], [92], the following phase change equations are expected to occur during the formation of $\mathrm{Fe}_{3} \mathrm{O}_{4}$ phase in the solvothermal process.

Ionization in solvent:

$$
\mathrm{Fe}\left(\mathrm{C}_{5} \mathrm{H}_{7} \mathrm{O}_{2}\right)_{3} \rightarrow \mathrm{Fe}^{3+}+3\left(\mathrm{C}_{5} \mathrm{H}_{7} \mathrm{O}_{2}\right)
$$

Phase transformations during synthesis:

$$
\begin{aligned}
& \mathrm{Fe}^{3+}+3\left(\mathrm{OH}^{-}\right) \rightarrow \mathrm{Fe}(\mathrm{OH})_{3} \\
& \mathrm{Fe}(\mathrm{OH})_{3} \rightarrow \alpha-\mathrm{Fe}_{2} \mathrm{O}_{3} \\
& \alpha-\mathrm{Fe}_{2} \mathrm{O}_{3} \rightarrow \mathrm{Fe}_{3} \mathrm{O}_{4}
\end{aligned}
$$

Effect of Synthesis Time on Size of NPs

As stated previously, various phases were detected to be formed during the solvothermal synthesis. The formation of the spinel $\mathrm{Fe}_{3} \mathrm{O}_{4} \mathrm{NPs}$ after 24 hours has been characterized already in Figure 27. Here, materials synthesized after 2 and 48 hours have 
been characterized, seen in Figure 43. Figure 43 (a) is a low magnification image of the iron-based NPs after 2 hours of synthesis. These NPs are thought to be $\mathrm{Fe}(\mathrm{OH})_{3}$ phase, however they are too small for detailed phase analysis via Raman spectroscopy, as they are in the range of $1-2 \mathrm{~nm}$. In Figure 43 (b), a relatively higher magnification image shows that the NPs are well distributed over the surface of graphene. Finally, in Figure 43 (c) the lattice fringes can be seen on the NPs, and the FFT inset reveal the nature of the NPs to be crystalline.

After 2 hours of synthesis
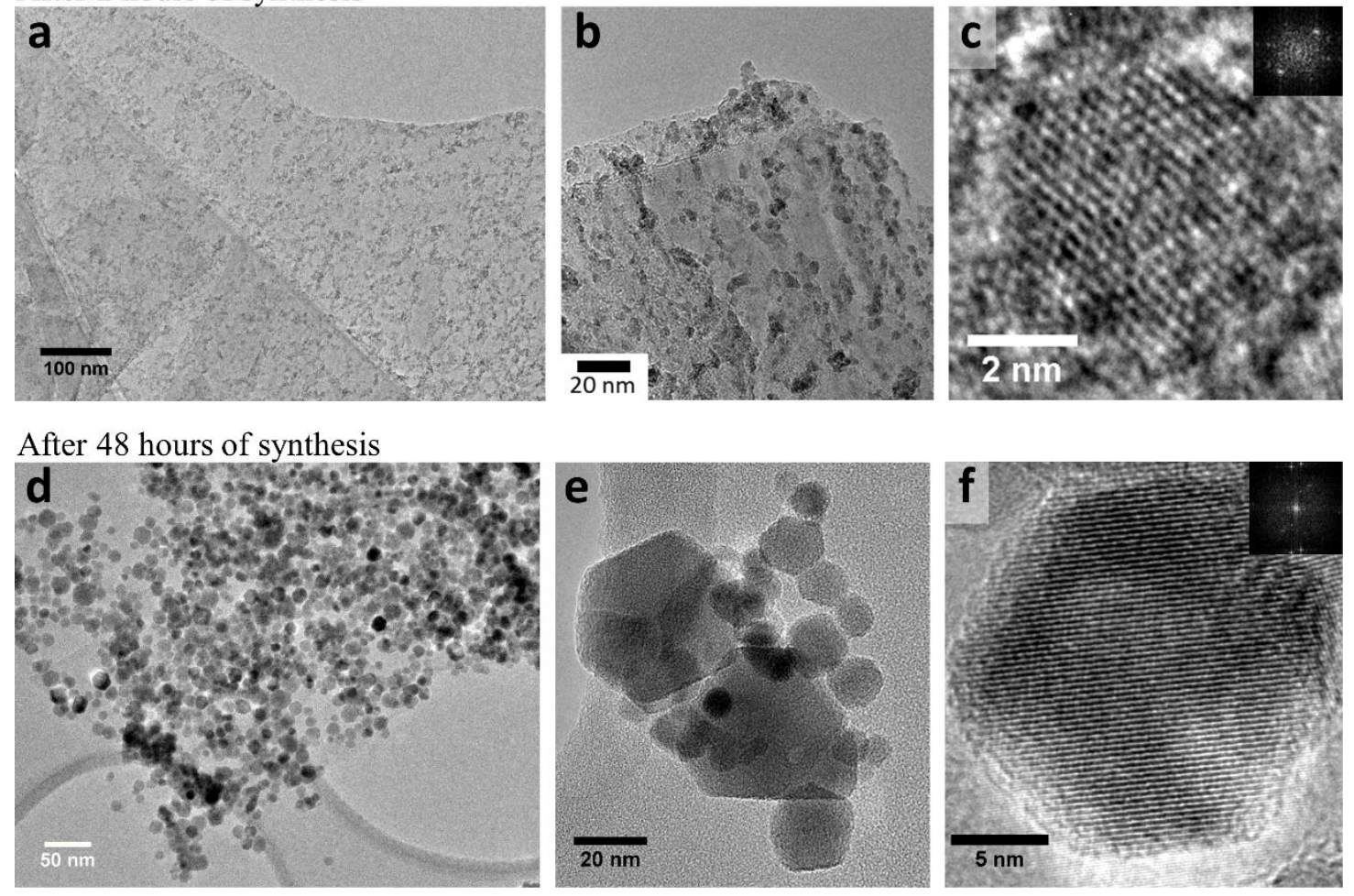

Figure 43. TEM images of graphene supported iron oxide NPs after $(\mathrm{a}-\mathrm{c}) 2$ hours and (b-f) 48 hours of synthesis time. (a) low magnification image of the NPs on graphene after just 2 hours of synthesis, (b) relatively higher magnification showing spread of NPs on the support, and (c) a closer look at a NPs with inset FFT revealing its crystalline nature. (d) low magnification image of NPs after 48 hours, (e) relatively higher magnification showing angular shape of NPs and (f) shows the lattice fringes and inset of FFT indicating the NPs are also crystalline. 
In Figure 43 (d), the specimen allowed to react for 48 hours were analyzed at lower magnifications, revealing uniform spread of NPs over the surface of graphene. This specimen is of $\mathrm{Fe}_{3} \mathrm{O}_{4}$ phase. In Figure 43 (e), a cluster of these NPs can be seen to have sharp edges with a hexagonal shape. A closer look at one such hexagonal NP, seen in Figure 43 (f) and the respective FFT in the inset shows that these NPs are also crystalline in nature. As expected, these NPs are much larger in size than those formed after 2 hours, in the range of $20-30 \mathrm{~nm}$, though some variations can be observed.

\section{Effect of Synthesis Temperature on Stability of $\mathrm{Fe}_{3} \mathrm{O}_{4}$ Phase}
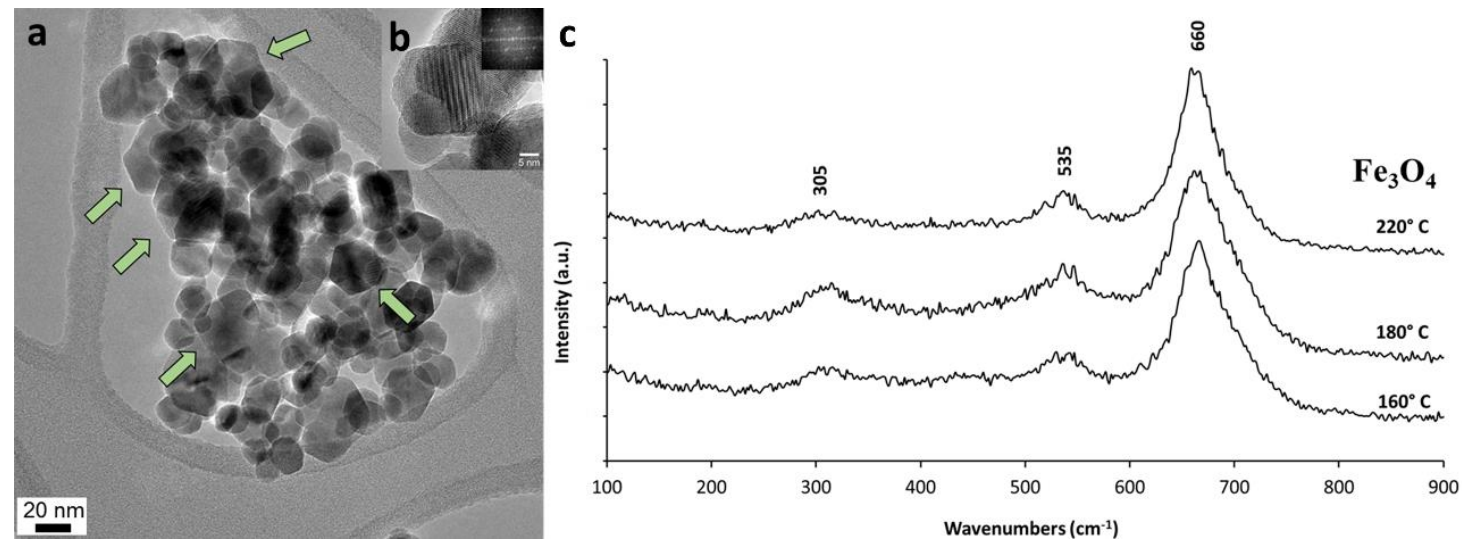

Figure 44. (a) $\mathrm{TEM}$ image of $\mathrm{Fe}_{3} \mathrm{O}_{4}$ formed at $220^{\circ} \mathrm{C}$ showing some particles with hexagonal shape. Arrows point towards some NPs where the shape is distinct. (b) HRTEM of a NP and inset FFT. (c) Raman spectra of the materials synthesized at $220^{\circ} \mathrm{C}$, $180^{\circ} \mathrm{C}$ and $160^{\circ} \mathrm{C}$.

To study the effect of temperature, keeping all other parameters the same, the autoclaves with the forming solution were allowed to react at higher and lower temperatures around $180^{\circ} \mathrm{C}$. Since the material formed at $180^{\circ} \mathrm{C}$ was characterized in Figure 27 , here materials fabricated at $220^{\circ} \mathrm{C}$ are examined seen in Figure 44 . In Figure 44 (a) iron oxide synthesized at $220^{\circ} \mathrm{C}$ were characterized, revealing NPs in the range of $10-$ 
$20 \mathrm{~nm}$. Arrows indicate NPs that have sharp edges in the shape of hexagons. One such hexagonal NP has been characterized further in Figure 44 (b) which, along with the FFT inset suggest the NPs are crystalline in nature. This material was characterized further using Raman spectroscopy, seen in Figure 44 (c) and compared with spectra of materials synthesized at $180^{\circ} \mathrm{C}$ and $160^{\circ} \mathrm{C}$, revealing that all specimens are spinel $\mathrm{Fe}_{3} \mathrm{O}_{4}$ phase NPs. To generate the electromagnetic results with the spinel $\mathrm{Fe}_{3} \mathrm{O}_{4}, 180^{\circ} \mathrm{C}$ was chosen as the appropriate synthesis temperature because of the uniformity in size of NPs produced.

\section{Effect of Acetone on NP Formation by Varying Synthesis Time and Temperature}

Thus far, ethanol was used as a solvent to synthesize the $\mathrm{Fe}_{3} \mathrm{O}_{4} \mathrm{NPs}$. Here, the effects of varying this solvent has been studied, seen in Figure 45.

Acetone was proven successful in producing TM NPs, hence it was used here and found to produce quasi-cubic NPs, as seen in Figure 45 (a), where the arrows point towards some NPs with clear shapes. These quasi-cubic NPs of around $40 \mathrm{~nm}$ appear to be surrounded with smaller, arbitrary-shaped NPs. The low image in Figure 45 (b) shows that these cubic NPs are present quite uniformly throughout the specimen. In Figure 45 (c), the HRTEM image with FFT inset shows that these NPs are single crystalline in nature. In Figure 45 (d), the Raman spectra of this specimen was compared to those made at different times up to 24 hours. Initially, the smaller $\mathrm{NPs}$ of $\mathrm{Fe}(\mathrm{OH})_{3}$ were detected, which undergo phase change around 5 hours to form $\alpha-\mathrm{Fe}_{2} \mathrm{O}_{3}$ phase NPs. In Figure 45 (e), a TEM image of a specimen made at $220^{\circ} \mathrm{C}$, after 24 hours synthesis is seen, showing mostly quasi-cubic NPs. The smaller, arbitrary-shaped NPs are absent in this specimen, suggesting they also grow into quasi-cubic shaped NPs under the right conditions. Figure 45 (f) is an HRTEM 
image of an edge of one such NPs showing lattice structures. This along with the inset FFT suggesting these NPs are also crystalline. The Raman spectra in Figure 45 (g) show that materials made at $220^{\circ} \mathrm{C}, 180^{\circ} \mathrm{C}$ and $160^{\circ} \mathrm{C}$ with acetone as solvent tend to form $\alpha-\mathrm{Fe}_{2} \mathrm{O}_{3}$ phase NPs.
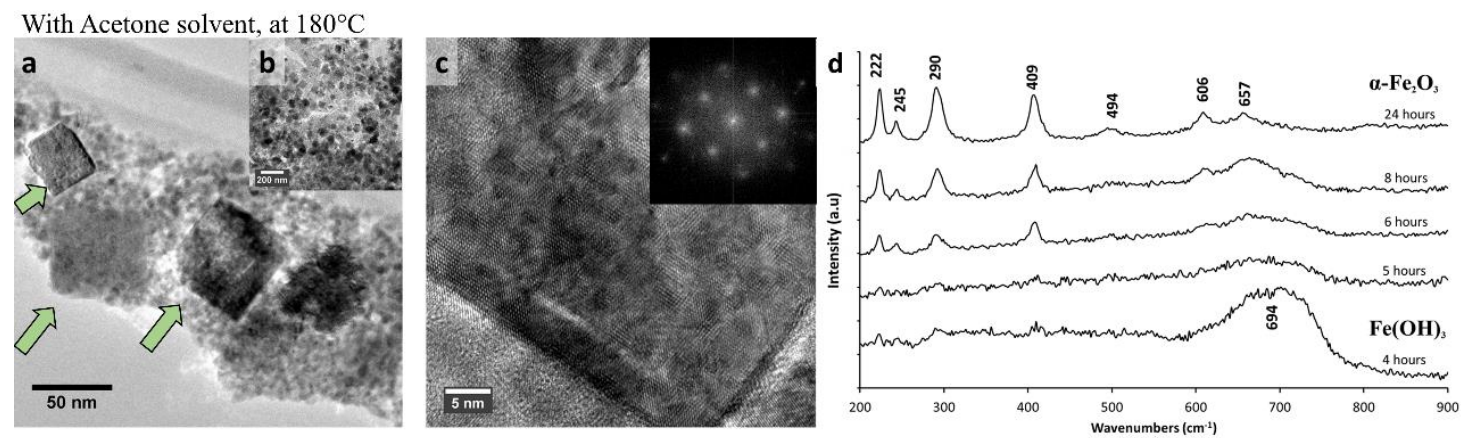

With Acetone solvent, at $220^{\circ} \mathrm{C}$
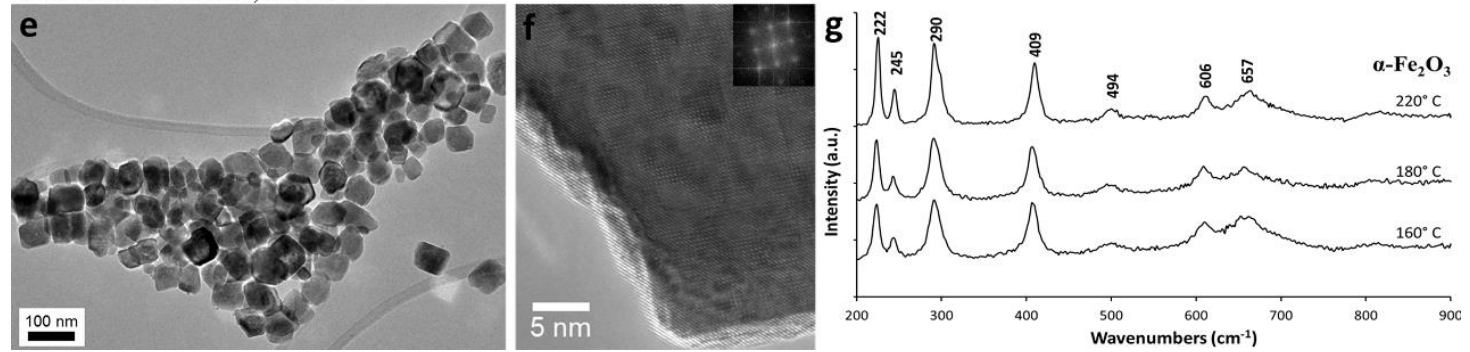

Figure 45. $(\mathrm{a}-\mathrm{b})$ Characterization of specimen made using acetone at $180^{\circ} \mathrm{C}$ (a) TEM image of the specimen after 24 hours showing formation of some quasi-cubic NPs, arrows point at prominent NPs. (b) low magnification image showing these cubic NPs are uniformly present in the specimen. (c) HRTEM image with FFT inset showing these NPs are crystalline in nature. (d) Raman spectra of the materials made at different times up to 24 hours. $(\mathrm{e}-\mathrm{g})$ Characterization of specimen made using acetone at $220^{\circ} \mathrm{C}$. (e) TEM image of specimen at $220^{\circ} \mathrm{C}$ after 24 hours showing mostly quasi-cubic NPs. (f) HRTEM image with inset suggesting these NPs are also crystalline. (g) Raman spectra showing at $220^{\circ} \mathrm{C}, 180^{\circ} \mathrm{C}$ and $160^{\circ} \mathrm{C}$ with acetone, $\alpha-\mathrm{Fe}_{2} \mathrm{O}_{3}$ phase NPs are formed.

While the quasi-cubic structures of $\alpha-\mathrm{Fe}_{2} \mathrm{O}_{3}$ are interesting, this phase of iron oxide has a rhombohedral crystal structure and is antiferromagnetic in nature. Hence, this phase cannot be used for the electromagnetic applications which are the focus of this application. 
Thus, it can be seen that the solvothermal process is flexible and allows the modification of NP crystal shape, size and structure by varying synthesis parameters. Using this process, we are able to make the material and phase of choice, in this case spinel $\mathrm{Fe}_{3} \mathrm{O}_{4}$.

\subsection{Conclusions and Future Work}

A new multiferroic material was developed for reversible control of magnetic moment by directly hybridizing $\mathrm{Fe} 3 \mathrm{O} 4$ NPs on graphene. A simple, environmentally friendly process was developed and optimized to produce spinel ferrites on graphene without surfactant or stabilizers. This material was used to make versatile electrode fabricated which can be used in both LIB and SC. With the new design of the electrode, a change in magnetic moment of the $\mathrm{Fe}_{3} \mathrm{O}_{4}$ NPs increased from $1 \%$ to $18 \%$ using a LIB to generate electric fields. In addition, reversible control of magnetic moment observed for the same electrode in a SC, which has not been reported previously.

This ability to synthesize and optimize the materials was developed after an understanding of the formation of NPs was studied, showing the various phase changes that the material underwent using the solvothermal process.

In the future, these electrodes can be optimized for testing in SCs and other devices. The electrode design can be further improved with other non-magnetic metal current collectors, with an idea to eliminate the use of $\mathrm{Cu}$ to prevent oxidation. It is also envisioned that this process can be further tweaked to develop superior spinel ferrites and perovskites that are susceptible to ME effect, while completely eliminating the need for PVDF. 


\section{Summary and Outlook}

The results presented in this thesis show a systematic approach towards developing, understanding and manipulation of material properties of TM and TMOs:

For the application of catalysis, Pd/Au NPs on carbon-supports were fabricated, characterized and optimized for effective removal of aqueous TCE. A green process was developed to make the catalyst which combined the sonochemical and solvothermal process to anchor transition metal (TM) NPs onto carbon supports. Since sustainability was a key factor for synthesis and application, GAC was found to be the preferred support for catalyst. The catalyst made in this project produced twice the rate if reaction when compared to corresponding commercial catalysts. This catalyst can be modified as per the need and optimized for other contaminants.

The methodology developed for the carbon-supported TM NPs was extended to carbon-supported TMO NPs. Showing the adaptability of this methodology, a different application was chosen for the carbon-supported TMO NPs. Thus, for the application of electromagnetism, $\mathrm{Fe}_{3} \mathrm{O}_{4}$ NPs hybridized on graphene were developed with an intent to increase the control of magnetism in electric fields. This new multiferroic material was developed by modifying the previously established green process with emphasis on the solvothermal process. The produced spinel ferrite was used to make a versatile electrode which was used in both LIBs and SCs. With this electrode, an increase from $1 \%$ to $18 \%$ in reversible change of magnetic moment was observed when tested in a LIB and a $2 \%$ change was observed in a SC. Further modifications to electrode and material can lead to complete on-off magnetism in materials. 
While the materials studied in this research are specific to the particular applications, the core of this dissertation is the methodology and systematic analysis of the material systems that was developed and modified as per the application needs. The methodical and structured approach presented here can be extended to similar materials for a variety of applications, for instance, the strategies applied for the Pd/Au NPs system can be extended to metals such as $\mathrm{Pt}, \mathrm{Cu}, \mathrm{Zn}$, and $\mathrm{Ag}$ NPs etc. while the $\mathrm{Fe}_{3} \mathrm{O}_{4} \mathrm{NPs}$ can be extended to spinel such as $\gamma-\mathrm{Fe}_{2} \mathrm{O}_{3}, \mathrm{Mn}_{3} \mathrm{O}_{4}$ and $\mathrm{Co}_{3} \mathrm{O}_{4}$ NPs etc. It is envisioned that this approach and strategy will contribute towards the future optimization of similar material systems for a multitude of applications. 


\section{References}

[1] R. J. D. Tilley and Wiley InterScience (Online service), Colour and the optical properties of materials : an exploration of the relationship between light, the optical properties of materials and colour. Wiley, 2011.

[2] U. Ndagi, N. Mhlongo, and M. E. Soliman, "Metal complexes in cancer therapy an update from drug design perspective," Drug Des. Devel. Ther., vol. 11, pp. 599-616, 2017.

[3] Q.-L. Zhou, "Transition-Metal Catalysis and Organocatalysis: Where Can Progress Be Expected?," Angew. Chemie Int. Ed., vol. 55, no. 18, pp. 5352-5353, Apr. 2016.

[4] S. Kaur-Ghumaan, A. Sakthivel, D. T. Masram, and S. Malaichamy, Electronic and magnetic properties of transition and inner transition elements and their complexes. Nova Science Publishers, Incorporated, 2017, 2017.

[5] B. P. Chaplin et al., "Critical Review of Pd-Based Catalytic Treatment of Priority Contaminants in Water," Environ. Sci. Technol, vol. 46, no. February, pp. 36553670, 2012.

[6] H. He, Y. Zhong, X. Liang, W. Tan, J. Zhu, and C. Y. Wang, "Natural magnetite: An efficient catalyst for the degradation of organic contaminant," Sci. Rep., vol. 5, pp. 1-10, 2015.

[7] S. Dasgupta et al., "Intercalation-driven reversible control of magnetism in bulk ferromagnets," Adv. Mater., vol. 26, no. 27, pp. 4639-4644, 2014.

[8] G. Wei et al., "Reversible control of the magnetization of spinel ferrites based electrodes by lithium-ion migration," Sci. Rep., vol. 7, no. 1, p. 12554, 2017.

[9] S. Dasgupta et al., "Toward On-and-Off Magnetism: Reversible Electrochemistry to Control Magnetic Phase Transitions in Spinel Ferrites," Adv. Funct. Mater., vol. 26, no. 41, pp. 7507-7515, 2016.

[10] G. Wei et al., "Reversible control of the magnetization of $\mathrm{Fe}_{3} \mathrm{O}_{4}$ via lithium ions," RSC Adv., vol. 7, no. 5, pp. 2644-2649, 2017.

[11] F. Matsukura, Y. Tokura, and H. Ohno, "Control of magnetism by electric fields," Nat. Nanotechnol., vol. 10, no. 3, pp. 209-220, 2015.

[12] W. Ji et al., "First-principles study of small Pd-Au alloy clusters on graphene," RSC Adv., vol. 4, no. 99, pp. 55781-55789, Oct. 2014.

[13] E. Lam and J. H. T. Luong, "Carbon materials as catalyst supports and catalysts in the transformation of biomass to fuels and chemicals," ACS Catal., vol. 4, no. 10, pp. 3393-3410, 2014.

[14] J. Huo et al., "Stability of Pd nanoparticles on carbon-coated supports under 
hydrothermal conditions," Catal. Sci. Technol., vol. 8, no. 4, pp. 1151-1160, 2018.

[15] I. Roche, E. Chainet, M. Chatenet, and J. Vondrak, "Carbon-supported manganese oxide nanoparticles as electrocatalysts for the Oxygen Reduction Reaction (ORR) in alkaline medium: Physical characterizations and ORR mechanism," J. Phys. Chem. C, vol. 111, no. 3, pp. 1434-1443, 2007.

[16] K. Guo, Y. Zhang, Q. Shi, and Z. Yu, "The Effect of Carbon-Supported Nickel Nanoparticles in the Reduction of Carboxylic Acids for in Situ Upgrading of Heavy Crude Oil," Energy and Fuels, vol. 31, no. 6, pp. 6045-6055, 2017.

[17] K. Meduri et al., "Palladium and gold nanoparticles on carbon supports as highly efficient catalysts for effective removal of trichloroethylene," J. Mater. Res., vol. 33, no. 16, pp. 2404-2413, 2018.

[18] K. Meduri, A. Barnum, G. O. B. Johnson, P. G. Tratnyek, and J. Jiao, "Characterization of Palladium and Gold Nanoparticles on Granular Activated Carbon as an Efficient Catalyst for Hydrodechlorination of Trichloroethylene," Microsc. Microanal., vol. 22, no. Suppl 3, pp. 332-333, 2016.

[19] K. Meduri, C. Stauffer, T. Lindner, G. O. B. Johnson, P. G. Tratnyek, and J. Jiao, "Effect of Synthesis Temperature on the Formation GAC supported Pd and Au NPs," Microsc. Microanal., vol. 23, no. Suppl 1, pp. 1916-1917, 2017.

[20] C. Stauffer, K. Meduri, G. O'Brien Johnson, P. G. Tratnyek, and J. Jiao, "Effect of Synthesis Time of Carbon Supported Pd/Au NPs on TCE degradation," Microsc. Microanal., vol. 24, no. S1, pp. 1802-1803, 2018.

[21] K. Meduri, C. Stauffer, G. O. Johnson, P. G. Tratnyek, and J. Jiao, "Electron Microscopy Characterization of the Synergistic Effects between Pd, Au NPs, and Their Graphene Support," Microsc. Microanal., vol. 24, no. S1, pp. 1888-1889, 2018.

[22] D. W. Clark and D. W. Briar, "What is Ground Water?"

[23] B. J. S. Zogorski et al., "The Quality of Our Nation's Waters: Volatile Organic Compounds in the Nation's Ground Water and Drinking-Water Supply Wells," 2006.

[24] H. Perlman and R. M. Waller, "Contaminants Found in Groundwater, USGS Water Science School," U.S. Department of the Interior, U.S. Geological Survey, 2016. [Online]. Available: https://water.usgs.gov/edu/groundwatercontaminants.html. [Accessed: 25-Feb-2018].

[25] M. J. Moran, J. S. Zogorski, and P. J. Squillace, "Chlorinated solvents in groundwater of the United States," Environ. Sci. Technol., vol. 41, no. 1, pp. 74 81, 2007.

[26] J. Cotruvo, J. K. Fawell, M. Giddings, P. Jackson, Y. Magara, and E. Ohanian, "Trichloroethene in Drinking-water, WHO Guidelines for Drinking-water 
Quality,”2005.

[27] "Substance Priority List 2017, Agency for Toxic Substances \&amp; Disease Registry," Agency for Toxic Substances and Disease Registry, 2017. [Online]. Available: https://www.atsdr.cdc.gov/spl/index.html. [Accessed: 25-Feb-2018].

[28] O. US EPA, "National Primary Drinking Water Regulations, 2017,” 2017.

[29] S. Li, Y. L. Fang, C. D. Romanczuk, Z. Jin, T. Li, and M. S. Wong, "Establishing the trichloroethene dechlorination rates of palladium-based catalysts and ironbased reductants," Appl. Catal. B Environ., vol. 125, pp. 95-102, 2012.

[30] N. Munakata and M. Reinhard, "Palladium-catalyzed aqueous hydrodehalogenation in column reactors: Modeling of deactivation kinetics with sulfide and comparison of regenerants," Appl. Catal. B Environ., vol. 75, no. 1-2, pp. 1-10, 2007.

[31] G. Gertrude, "Hydrogenation of mono-and disaccharides to polyols," US2868847 A, 1959.

[32] E. Díaz, S. Ordóñez, R. F. Bueres, E. Asedegbega-Nieto, and H. Sastre, "Highsurface area graphites as supports for hydrodechlorination catalysts: Tuning support surface chemistry for an optimal performance," Appl. Catal. B Environ., vol. 99, no. 1-2, pp. 181-190, 2010.

[33] S. Ordóñez, E. Díaz, R. F. Bueres, E. Asedegbega-Nieto, and H. Sastre, "Carbon nanofibre-supported palladium catalysts as model hydrodechlorination catalysts," J. Catal., vol. 272, no. 1, pp. 158-168, 2010.

[34] M. Zhang, D. B. Bacik, C. B. Roberts, and D. Zhao, "Catalytic hydrodechlorination of trichloroethylene in water with supported CMC-stabilized palladium nanoparticles," Water Res., vol. 47, no. 11, pp. 3706-3715, 2013.

[35] A. Schrage, "Preparation of carbon supported palladium catalysts," US3736266A, 1973.

[36] B. Daniel L and C. D. Keith, "Process for producing palladium on carbon catalysts," US3138560A, 1964.

[37] R. Mozingo, "Palladium Catalysts," Org. Synth., vol. 26, no. September, p. 77, 1946.

[38] J. Cookson and B. J. Cookson, "The preparation of palladium nanoparticles," Platin. Met. Rev., vol. 56, no. 2, pp. 83-98, 2012.

[39] P. Riesz, D. Berdahi, and C. L. Christman, "Free Radical Generation by Ultrasound in Aqueous and Nonaqueous Solutions," Environ. Health Perspect., vol. 64, pp. 233-252, 1985.

[40] R. A. Caruso, M. Ashokkumar, and F. Grieser, "Sonochemical formation of gold 
sols," Langmuir, vol. 18, no. 21, pp. 7831-7836, 2002.

[41] A. Tauber, G. Mark, H. Schuchmann, and C. Von Sonntag, "Sonolysis of tert-butyl alcohol in aqueous solution," J. Chem. Soc., Perkin Trans. 2, vol. 0, pp. 1129$1135,1999$.

[42] J. H. Bang and K. S. Suslick, "Applications of ultrasound to the synthesis of nanostructured materials," Adv. Mater., vol. 22, no. 10, pp. 1039-1059, 2010.

[43] Y. Mizukoshi, T. Fujimoto, Y. Nagata, R. Oshima, and Y. Maeda, "Characterization and Catalytic Activity of Core-Shell Structured Gold/Palladium Bimetallic Nanoparticles Synthesized by the Sonochemical Method," J. Phys. Chem. B, vol. 104, no. 25, pp. 6028-6032, 2000.

[44] Y. Nagata, Y. Mizukoshi, K. Okitsu, and Y. Maeda, "Sonochemical formation of gold particles in aqueous solution," Radiat. Res., vol. 146, no. 3, pp. 333-338, 1996.

[45] T. Akita, N. Hase, N. Taguchi, S. Tanaka, M. Kohyama, and F. Hori, "Analytical TEM study of the core-shell structure of Au-Pd nano-particles prepared by sonochemical technique," J. Phys. Conf. Ser., vol. 100, no. 1, pp. 1-4, 2008.

[46] B. Gersten, "Solvothermal Synthesis of Nanoparticles," Chemfiles, 2004. [Online]. Available: https://www.sigmaaldrich.com/content/dam/sigma-

aldrich/articles/chemfiles/volume5a13/Solvothermal_Synthesis_of_Nanoparticles. pdf. [Accessed: 09-Jul-2018].

[47] G. Demazeau, "Solvothermal processes: Definition, Key factors governing the involved chemical reactions and new trends," Zeitschrift fur Naturforsch. - Sect. B J. Chem. Sci., vol. 65, no. 8, pp. 999-1006, 2010.

[48] M. Reinhard and C. LeBron, "In Situ Catalytic Groundwater Treatment Using Palladium Catalysts and Horizontal Flow Treatment Wells (Cost and Performance Report)," 2007.

[49] J. K. Edwards, A. Thomas, A. F. Carley, A. a. Herzing, C. J. Kiely, and G. J. Hutchings, "Au-Pd supported nanocrystals as catalysts for the direct synthesis of hydrogen peroxide from $\mathrm{H} 2$ and O2," Green Chem., vol. 10, no. 4, p. 388, 2008.

[50] Y. Hernandez et al., "High-yield production of graphene by liquid-phase exfoliation of graphite," Nat. Nanotechnol., vol. 3, no. 9, pp. 563-568, 2008.

[51] J. L. Hart et al., "Direct Detection Electron Energy-Loss Spectroscopy: A Method to Push the Limits of Resolution and Sensitivity," Sci. Rep., vol. 7, no. 1, pp. 1-14, 2017.

[52] M. O. Nutt, J. B. Hughes, and M. S. Wong, "Designing Pd-on-Au Bimetallic Nanoparticle Catalysts for Trichloroethene Hydrodechlorination," Environ. Sci. Technol., vol. 39, no. 5, pp. 1346-1353, 2005. 
[53] M. O. Nutt, K. N. Heck, P. Alvarez, and M. S. Wong, "Improved Pd-on-Au bimetallic nanoparticle catalysts for aqueous-phase trichloroethene hydrodechlorination," Appl. Catal. B Environ., vol. 69, no. 1-2, pp. 115-125, 2006.

[54] A. C. Ferrari et al., "Raman Spectrum of Graphene and Graphene Layers," vol. 187401, no. NOVEMBER, pp. 1-4, 2006.

[55] R. Petro, P. Borodulin, T. E. Schlesinger, and M. Schlesinger, "Liquid Exfoliated Graphene: A Practical Method for Increasing Loading and Producing Thin Films," ECS J. Solid State Sci. Technol., vol. 5, no. 2, pp. P36-P40, 2016.

[56] Y. Mizukoshi, K. Okitsu, Y. Maeda, T. A. Yamamoto, R. Oshima, and Y. Nagata, "Sonochemical preparation of bimetallic nanoparticles of gold/palladium in aqueous solution," J. Phys. Chem. B, vol. 101, no. 36, pp. 7033-7037, 1997.

[57] A. Patil et al., "Granular Activated Carbon (GAC) Fact Sheet," Wat. Qual. Assoc., pp. 1-6, 2016.

[58] H. Jin, S. Park, and J. M. Lee, "The shape-selectivity of activated carbon fibers as a palladium catalyst support," Carbon N. Y., vol. 34, no. 3, pp. 429-431, 1996.

[59] G. V. Lowry and M. Reinhard, "Hydrodehalogenation of 1- to 3-Carbon Halogenated Organic Compounds in Water Using a Palladium Catalyst and Hydrogen Gas," Environ. Sci. Technol., vol. 33, no. 650, pp. 1905-1910, 1999.

[60] R. Ferrando, J. Jellinek, and R. L. Johnston, "Nanoalloys: From theory to applications of alloy clusters and nanoparticles," Chem. Rev., vol. 108, no. 3, pp. 845-910, 2008.

[61] R. Larsen, S. Ha, J. Zakzeski, and R. I. Masel, "Unusually active palladium-based catalysts for the electrooxidation of formic acid," Journal of Power Sources, vol. 157, no. 1. pp. 78-84, 2006.

[62] L. Gorton and T. Svensson, "An investigation of the influences of the background material and layer thickness of sputtered palladium/gold on carbon electrodes for the amperometric determination of hydrogen peroxide," J. Mol. Catal., vol. 38, no. 1-2, pp. 49-60, Nov. 1986.

[63] A. Bianco et al., "All in the graphene family - A recommended nomenclature for two-dimensional carbon materials," Carbon N. Y., vol. 65, pp. 1-6, 2013.

[64] F. Rodriguez-Reinoso, J. M. Martin-Martinez, C. Prado-Burguete, and B. McEnaney, "A standard adsorption isotherm for the characterization of activated carbons," J. Phys. Chem., vol. 91, no. 3, pp. 515-516, 1987.

[65] B. Fang, N. K. Chaudhari, M.-S. Kim, J. H. Kim, and J.-S. Yu, "Homogeneous Deposition of Platinum Nanoparticles on Carbon Black for Proton Exchange Membrane Fuel Cell," J. Am. Chem. Soc., vol. 131, no. 42, pp. 15330-15338, 2009. 
[66] A. Eshghi, "Palladium nanoparticles supported on carbon black powder as an effective anodic catalyst for application in a direct glucose alkaline fuel cell," vol. 1, pp. 11-17, 2016.

[67] M. Bron, "Carbon black supported gold nanoparticles for oxygen electroreduction in acidic electrolyte solution," Journal of Electroanalytical Chemistry, vol. 624, no. 1-2. pp. 64-68, 2008.

[68] H. Sato et al., "Comprehensive study of CoFeB-MgO magnetic tunnel junction characteristics with single- and double-interface scaling down to $1 \mathrm{X}$ nm," Tech. Dig. - Int. Electron Devices Meet. IEDM, pp. 60-63, 2013.

[69] D. Chiba, S. Fukami, K. Shimamura, N. Ishiwata, K. Kobayashi, and T. Ono, "Electrical control of the ferromagnetic phase transition in cobalt at room temperature," Nat. Mater., vol. 10, no. 11, pp. 853-856, 2011.

[70] A. Molinari et al., "Hybrid supercapacitors for reversible control of magnetism," Nat. Commun., vol. 8, no. May, p. 15339, 2017.

[71] E. Antolini, "Catalysts for direct ethanol fuel cells," vol. 170, pp. 1-12, 2007.

[72] J. Walter, H. Wang, B. Luo, C. D. Frisbie, and C. Leighton, "Electrostatic versus Electrochemical Doping and Control of Ferromagnetism in Ion-Gel-Gated Ultrathin La0.5Sr0.5CoO3- $\delta$," ACS Nano, vol. 10, no. 8, pp. 7799-7810, 2016.

[73] W. Qian et al., "Surfactant-free hybridization of transition metal oxide nanoparticles with conductive graphene for high-performance supercapacitor," Green Chem., vol. 14, p. 371, 2012.

[74] G. Kucinskis, G. Bajars, and J. Kleperis, "Graphene in lithium ion battery cathode materials: A review,” J. Power Sources, vol. 240, pp. 66-79, 2013.

[75] D. Chen, J. Li, and D. Chen, "Graphene-based materials in electrochemistry," pp. 3157-3180, 2010.

[76] G. Jo, M. Choe, S. Lee, W. Park, Y. H. Kahng, and T. Lee, "The application of graphene as electrodes in electrical and optical devices," Nanotechnology, vol. 23, no. 11, p. 112001, 2012.

[77] J. Vega-Chacón, G. Picasso, L. Avilés-Félix, and M. J. Jr, "Influence of synthesis experimental parameters on the formation of magnetite nanoparticles prepared by polyol method," Adv. Nat. Sci. Nanosci. Nanotechnol., vol. 7, no. 1, p. 15014, 2016.

[78] Y. S. Kang, S. Risbud, J. F. Rabolt, and P. Stroeve, "Synthesis and Characterization of Nanometer-Size $\mathrm{Fe}_{3} \mathrm{O}_{4}$ and $\gamma$-Fe ${ }_{2} \mathrm{O}_{3}$ Particles," Chem. Mater., vol. 8, no. 9, pp. 2209-2211, 1996.

[79] P. Ou, G. Xu, C. Xu, Y. Zhang, X. Hou, and G. Han, "Synthesis and characterization of magnetite nanoparticles by a simple solvothermal method," 
Mater. Sci., vol. 28, no. 4, 2010.

[80] R. V. Kumar, Y. Koltypin, X. N. Xu, Y. Yeshurun, A. Gedanken, and I. Felner, "Fabrication of magnetite nanorods by ultrasound irradiation," J. Appl. Phys., vol. 89, no. 11 I, pp. 6324-6328, 2001.

[81] L. Néel, "Experimental evidence of ferromagnetism and antiferromagnetism," Ann Inst Fourier, vol. 1, pp. 163-183, 1949.

[82] C. De Las Casas and W. Li, "A review of application of carbon nanotubes for lithium ion battery anode material," J. Power Sources, vol. 208, pp. 74-85, 2012.

[83] X. J. S. Dahn J.R, Zheng T, Liu Y, "Mechanisms for Lithium Insertion in Carbanaceous Materials," Amercican Assoc. Adv. Sci., vol. 270, no. 5326, pp. 590593, 1995.

[84] A. Molinari, H. Hahn, and R. Kruk, "Voltage-Controlled On/Off Switching of Ferromagnetism in Manganite Supercapacitors," Adv. Mater., vol. 30, no. 1, pp. 16, 2018.

[85] Y. Sun, N. Liu, and Y. Cui, "Promises and challenges of nanomaterials for lithium-based rechargeable batteries," Nat. Energy, vol. 1, no. 7, p. 16071, 2016.

[86] M. Halper and J. Ellenbogen, "Supercapacitors: A brief overview," Rep. No. MP 05W0000272, ..., no. March, p. Report No. MP 05W0000272, 1-29, 2006.

[87] E. Frackowiak, K. Jurewicz, K. Szostak, S. Delpeux, and F. Béguin, "Nanotubular materials as electrodes for supercapacitors," Fuel Process. Technol., vol. 77-78, pp. 213-219, 2002.

[88] L. V. Gasparov, D. B. Tanner, D. B. Romero, H. Berger, G. Margaritondo, and L. Forró, "Infrared and Raman studies of the Verwey transition in magnetite," Phys. Rev. B - Condens. Matter Mater. Phys., vol. 62, no. 12, pp. 7939-7944, 2000.

[89] A. Zoppi, C. Lofrumento, E. M. Castellucci, and M. G. Migliorini, "The Raman Spectrum of Hematite: Possible Indicator for a Compositional or Firing Distinction Among Terra Sigillata Wares," Ann. Chim., vol. 95, 2005.

[90] D. Bersani, P. P. Lottici, and a Montenero, "Micro-Raman Investigation of Iron Oxide Films and Powders Produced by Sol - Gel Syntheses," J. Raman Spectrosc., vol. 30, no. February 1998, pp. 355-360, 1999.

[91] V. D. Nithya and N. Sabari Arul, "Progress and development of Fe3O4 electrodes for supercapacitors," J. Mater. Chem. A, vol. 4, no. 28, pp. 10767-10778, 2016.

[92] A. G. Nene, M. Takahashi, and P. R. Somani, "Fe 3 O 4 and Fe Nanoparticles by Chemical Reduction of Fe ( acac ) 3 by Ascorbic Acid : Role of Water," no. March, pp. 20-28, 2016. 